\title{
Weak convergence rates of spectral Galerkin approximations for SPDEs with nonlinear diffusion coefficients
}

\author{
Daniel Conus, Arnulf Jentzen, and Ryan Kurniawan \\ Lehigh University, USA and ETH Zurich, Switzerland
}

May 2, 2018

\begin{abstract}
Strong convergence rates for (temporal, spatial, and noise) numerical approximations of semilinear stochastic evolution equations (SEEs) with smooth and regular nonlinearities are well understood in the scientific literature. Weak convergence rates for numerical approximations of such SEEs have been investigated for about two decades and are far away from being well understood: roughly speaking, no essentially sharp weak convergence rates are known for parabolic SEEs with nonlinear diffusion coefficient functions; see Remark 2.3 in [A. Debussche, Weak approximation of stochastic partial differential equations: the nonlinear case, Math. Comp. 80 (2011), no. 273, 89-117] for details. In this article we solve the weak convergence problem emerged from Debussche's article in the case of spectral Galerkin approximations and establish essentially sharp weak convergence rates for spatial spectral Galerkin approximations of semilinear SEEs with nonlinear diffusion coefficient functions. Our solution to the weak convergence problem does not use Malliavin calculus. Rather, key ingredients in our solution to the weak convergence problem emerged from Debussche's article are the use of appropriately modified versions of the spatial Galerkin approximation processes and applications of a mild Itô type formula for solutions and numerical approximations of semilinear SEEs. This article solves the weak convergence problem emerged from Debussche's article merely in the case of spatial spectral Galerkin approximations instead of other more complicated numerical approximations. Our method of proof extends, however, to a number of other kinds of spatial and temporal numerical approximations for semilinear SEEs.
\end{abstract}

\section{Contents}

1 Introduction

1.1 Sketch of the proof of the main weak convergence result . . . . . . . . . . .

1.2 Examples . . . . . . . . . . . . . . . . . . . . 1.2.1 Parabolic Anderson model and nonlinear heat-type SPDEs . . . . . 1.2.2 A Cahn-Hilliard-Cook type equation . . . . . . . . . . .

1.3 Notation . . . . . . . . . . . . . . . . . . . . . . . . . . .

1.4 Setting . . . . . . . . . . . . . . . . . . . . . . . 9

1.5 Auxiliary lemmas . . . . . . . . . . . . . . . . . 10 
2 Weak convergence for Galerkin projections of SEEs

2.1 Setting . . . . . . . . . . . . . . . . . . . 10

2.2 A weak convergence result . . . . . . . . . . . . . . . 10

3 Weak convergence for Galerkin approximations of SEEs with mollified nonlinearities

3.1 Regularity properties for solutions of infinite dimensional Kolmogorov equations in Hilbert spaces . . . . . . . . . . . . . . . . . . 13

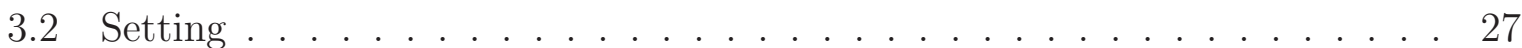

3.3 Weak convergence results . . . . . . . . . . . . . . . . 28

4 Strong convergence of mollified solutions for SEEs 43

4.1 Setting . . . . . . . . . . . . . . . . . . . . . . 43

4.2 A strong convergence result . . . . . . . . . . . . . . . 43

5 Weak convergence for Galerkin approximations of SEEs 45

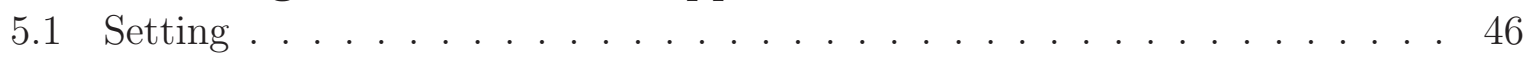

5.2 A weak convergence result . . . . . . . . . . . . . . 46

6 Weak convergence rates for SEEs 51

7 Lower bounds for the weak error of Galerkin approximations for SEEs 52

7.1 Setting . . . . . . . . . . . . . . . . . . 53

7.2 Lower bounds for the weak error . . . . . . . . . . . . . . . . . 53

\section{Introduction}

Both strong and numerically weak convergence rates for numerical approximations of finite dimensional stochastic ordinary differential equations (SODEs) with smooth and regular nonlinearities are well understood in the literature; see, e.g., the monographs Kloeden \& Platen [31] and Milstein [37]. The situation is different in the case of possibly infinite dimensional semilinear stochastic evoluation equations (SEEs). While strong convergence rates for (temporal, spatial, and noise) numerical approximations of semilinear SEEs with smooth and regular nonlinearities are well understood in the scientific literature, weak convergence rates for numerical approximations of such SEEs have been investigated since about 14 years ago and are far away from being well understood: roughly speaking, no essentially sharp weak convergence rates are known for parabolic SEEs with nonlinear diffusion coefficient functions (see Remark 2.3 in Debussche [19] for details). In this article we solve the weak convergence problem emerged from Debussche's article in the case of spectral Galerkin approximations and establish essentially sharp weak convergence rates for spatial spectral Galerkin approximations of semilinear SEEs with nonlinear diffusion coefficient functions. To illustrate the weak convergence problem emerged from Debussche's article and our solution to the problem we consider the following setting as a special case of our general setting in Section 5 below. Let $\left(H,\langle\cdot, \cdot\rangle_{H},\|\cdot\|_{H}\right)$ and $\left(U,\langle\cdot, \cdot\rangle_{U},\|\cdot\|_{U}\right)$ be separable $\mathbb{R}$-Hilbert spaces, let $T \in(0, \infty)$, let $\left(\Omega, \mathcal{F}, \mathbb{P},\left(\mathcal{F}_{t}\right)_{t \in[0, T]}\right)$ be a stochastic basis, let $\left(W_{t}\right)_{t \in[0, T]}$ be an $\operatorname{Id}_{U^{-}}$ cylindrical $\left(\Omega, \mathcal{F}, \mathbb{P},\left(\mathcal{F}_{t}\right)_{t \in[0, T]}\right)$-Wiener process, let $\left(e_{n}\right)_{n \in \mathbb{N}} \subseteq H$ be an orthonormal basis of $H$, let $\left(\lambda_{n}\right)_{n \in \mathbb{N}} \subseteq(0, \infty)$ be an increasing sequence, let $A: D(A) \subseteq H \rightarrow H$ be a closed linear operator such that $D(A)=\left\{v \in H: \sum_{n \in \mathbb{N}}\left|\lambda_{n}\left\langle e_{n}, v\right\rangle_{H}\right|^{2}<\infty\right\}$ and $\forall n \in \mathbb{N}: A e_{n}=-\lambda_{n} e_{n}$, let $\left(H_{r},\langle\cdot, \cdot\rangle_{H_{r}},\|\cdot\|_{H_{r}}\right), r \in \mathbb{R}$, be a family of interpolation spaces associated to $-A$ (cf., e.g., [44, Section 3.7]), let $\iota \in[0,1 / 4], \xi \in H_{\iota}, \gamma \in[0,1 / 2]$, 
and let $F \in \cap_{r<\iota-\gamma} C_{b}^{4}\left(H_{\iota}, H_{r}\right), B \in \cap_{r<\iota-\gamma / 2} C_{b}^{4}\left(H_{\iota}, H S\left(U, H_{r}\right)\right)$. For two $\mathbb{R}$-Hilbert spaces $\left(V_{1},\langle\cdot, \cdot\rangle_{V_{1}},\|\cdot\|_{V_{1}}\right)$ and $\left(V_{2},\langle\cdot, \cdot\rangle_{V_{2}},\|\cdot\|_{V_{2}}\right)$ we denote by $C_{b}^{4}\left(V_{1}, V_{2}\right)$ the $\mathbb{R}$-vector space of all four times continuously Fréchet differentiable functions from $V_{1}$ to $V_{2}$ with globally bounded derivatives and by $H S\left(V_{1}, V_{2}\right)$ the $\mathbb{R}$-Hilbert space of all Hilbert-Schmidt operators from $V_{1}$ to $V_{2}$. We also note that the hypothesis that $\left(H_{r},\langle\cdot, \cdot\rangle_{H_{r}},\|\cdot\|_{H_{r}}\right), r \in \mathbb{R}$, is a family of interpolation spaces associated to $-A$ ensures for all $r \in[0, \infty)$ that $H_{r}=D\left((-A)^{r}\right)$ and $H_{1}=D(A) \subseteq H=H_{0}$. The above assumptions imply (cf., e.g., Da Prato et al. [14, Proposition 3], Brzeźniak [8, Theorem 4.3], Van Neerven et al. 47, Theorem 6.2]) the existence of a continuous mild solution process $X:[0, T] \times \Omega \rightarrow H_{\iota}$ of the SEE

$$
d X_{t}=\left[A X_{t}+F\left(X_{t}\right)\right] d t+B\left(X_{t}\right) d W_{t}, \quad t \in[0, T], \quad X_{0}=\xi
$$

As an example for (1), we think of $H=U=L^{2}((0,1) ; \mathbb{R})$ being the $\mathbb{R}$-Hilbert space of equivalence classes of Lebesgue-Borel square integrable functions from $(0,1)$ to $\mathbb{R}$ and $A$ being an appropriate linear differential operator on $H$. In particular, in Subsection 1.2.1 we formulate the continuous version of the one-dimensional parabolic Anderson model as an example for (1) (in this example the parameter $\gamma$, which controls the regularity of the operators $F$ and $B$, satisfies $\gamma=1 / 2$ ) and in Subsection 1.2 .2 we formulate a fourth-order stochastic partial differential equation as an example for (11) (in this second example the parameter $\gamma$ satisfies $\gamma=1 / 4$ ).

Strong convergence rates for (temporal, spatial, and noise) numerical approximations for SEEs of the form (11) are well understood. Weak convergence rates for numerical approximations of SEEs of the form (11) have been investigated for about two decades; cf., e.g., [45, 24, 18, 20, 22, 25, 19, 32, 21, 35, 36, 33, 50, 34, 6, 48, 4, 5, 7, 49]. Except for Debussche \& De Bouard [18], Debussche [19], and Andersson \& Larsson [5], all of the above mentioned references assume, beside further assumptions, that the considered SEE is driven by additive noise. In Debussche \& De Bouard [18] weak convergence rates for the nonlinear Schrödinger equation, which dominant linear operator generates a group (see [18, Section 2]) instead of only a semigroup as in the general setting of the SEE (1), are analyzed. The method of proof in Debussche \& De Bouard [18] strongly exploits this property of the nonlinear Schrödinger equation (see [18, Section 5.2]). Therefore, the method of proof in 18] can, in general, not be used to establish weak convergence rates for the SEE (11). In Debussche's seminal article [19] (see also Andersson \& Larsson [5]), essentially sharp weak convergence rates for SEEs of the form (11) are established under the hypothesis that the second derivative of the diffusion coefficient $B$ satisfies the smoothing property that there exists a real number $L \in[0, \infty)$ such that for all $x, v, w \in H$ it holds that 1

$$
\left\|B^{\prime \prime}(x)(v, w)\right\|_{L(H)} \leq L\|v\|_{H_{-1 / 4}}\|w\|_{H_{-1 / 4}} .
$$

As pointed out in Remark 2.3 in Debussche [19], assumption (2) is a serious restriction for SEEs of the form (11). Roughly speaking, assumption (2) imposes that the second derivative of the diffusion coefficient function vanishes and thus that the diffusion coefficient function is affine linear. Remark 2.3 in Debussche [19] also asserts that assumption (2) is crucial in the weak convergence proof in [19], that assumption (21) is used in an essential way in Lemma 4.5 in [19], and that Lemma 4.5 in [19], in turn, is used at many points in the weak convergence proof in [19]. To the best of our knowledge, it remained an open problem to establish essentially sharp weak convergence rates for any type of temporal, spatial, or noise numerical approximation of the SEE (1) without imposing Debussche's assumption (2). In this article we solve this problem in the case of spatial

\footnotetext{
${ }^{1}$ Assumption (2) above slightly differs from the original assumption in [19] as we believe that there is a small typo in equation (2.5) in [19]; see inequality (4.3) in the proof of Lemma 4.5 in [19] for details.
} 
spectral Galerkin approximations for the SEE (1). This is the subject of the following theorem (Theorem 1.1), which follows immediately from Corollary 6.1 below.

Theorem 1.1. Assume the setting in the first paragraph of Section 1 , let $\varphi \in C_{b}^{4}\left(H_{\iota}, \mathbb{R}\right)$, let $\left(P_{N}\right)_{N \in \mathbb{N}} \subseteq L\left(H_{-1}\right)$ satisfy for all $N \in \mathbb{N}, v \in H$ that $P_{N}(v)=\sum_{n=1}^{N}\left\langle e_{n}, v\right\rangle_{H} e_{n}$, and for every $N \in \mathbb{N}$ let $X^{N}:[0, T] \times \Omega \rightarrow P_{N}(H)$ be a continuous mild solution of the $S E E$

$$
d X_{t}^{N}=\left[P_{N} A X_{t}^{N}+P_{N} F\left(X_{t}^{N}\right)\right] d t+P_{N} B\left(X_{t}^{N}\right) d W_{t}, \quad t \in[0, T], \quad X_{0}^{N}=P_{N}(\xi) .
$$

Then for every $\varepsilon \in(0, \infty)$ there exists a real number $C_{\varepsilon} \in[0, \infty)$ such that for all $N \in \mathbb{N}$ it holds that

$$
\left|\mathbb{E}\left[\varphi\left(X_{T}\right)\right]-\mathbb{E}\left[\varphi\left(X_{T}^{N}\right)\right]\right| \leq C_{\varepsilon} \cdot\left(\lambda_{N}\right)^{-(1-\gamma-\varepsilon)} .
$$

Let us add a few comments regarding Theorem 1.1. First, we would like to emphasize that in the general setting of Theorem 1.1, the weak convergence rate established in Theorem 1.1 can essentially not be improved. More specifically, in Corollary 7.5 in Section 7 below we give for every $\iota \in[0,1 / 4]$ and every $\gamma \in[0,1 / 2]$ examples of $A: D(A) \subseteq H \rightarrow H$, $\xi \in H_{\iota}, F \in \cap_{r<\iota-\gamma} C_{b}^{4}\left(H_{\iota}, H_{r}\right),\left(U,\langle\cdot, \cdot\rangle_{U},\|\cdot\|_{U}\right), B \in \cap_{r<\iota-\gamma / 2} C_{b}^{4}\left(H_{\iota}, H S\left(U, H_{r}\right)\right)$, and $\varphi \in C_{b}^{4}\left(H_{\iota}, \mathbb{R}\right)$ such that there exists a real number $C \in(0, \infty)$ such that for all $N \in \mathbb{N}$ it holds that

$$
\left|\mathbb{E}\left[\varphi\left(X_{T}\right)\right]-\mathbb{E}\left[\varphi\left(X_{T}^{N}\right)\right]\right| \geq C \cdot\left(\lambda_{N}\right)^{-(1-\gamma)} .
$$

In addition, we emphasize that in the setting of Theorem 1.1 it is well known (cf., e.g., Cox et al. [11, Corollary 3.3]) that for every $\varepsilon \in(0, \infty)$ there exists a real number $C_{\varepsilon} \in[0, \infty)$ such that for all $N \in \mathbb{N}$ it holds that

$$
\left(\mathbb{E}\left[\left\|X_{T}-X_{T}^{N}\right\|_{H_{\iota}}^{2}\right]\right)^{1 / 2} \leq C_{\varepsilon} \cdot\left(\lambda_{N}\right)^{-\left(\frac{1-\gamma}{2}-\varepsilon\right)} .
$$

The weak convergence rate $1-\gamma-\varepsilon$ established in Theorem 1.1 is thus twice the wellknown strong convergence rate $\frac{1-\gamma-\varepsilon}{2}$ in (6). Moreover, Theorem 1.1 is - to the best of our knowledge - the first result in the scientific literature which establishes an essentially sharp weak convergence rate for numerical approximations of the continuous version of the one-dimensional parabolic Anderson model (see Subsection 1.2.1 for details). We also would like to point out that the weak convergence result in Theorem 2.2 in Debussche [19] assumes that (2) holds (see (2.5) in [19]), that $B$ maps from $H$ to $L(H)$ (instead of from $H$ to $H S\left(U, H_{r}\right)$ for $\left.r \in(-\infty,-1 / 2)\right)$, and that $\varphi, F$, and $B$ are three times continuously Fréchet differentiable with globally bounded derivatives (instead of four times continuously Fréchet differentiable as in Theorem 1.1 above) but restricts to the irregular case $\gamma=$ $1 / 2$ in the above framework. The weak convergence result in Theorem 1.1 above does not assume (2) and does assume that $\varphi, F$, and $B$ are four times continuously Fréchet differentiable but also establishes essentially sharp weak convergence rates in the more regular cases $\gamma \in[0,1 / 2)$ such as in several cases of trace class noise. In the very regular case of finite dimensional SEEs it is typically assumed that $F$ and $B$ (and $\varphi$ ) are four times continuously differentiable (cf., e.g., Kloeden \& Platen [31, Theorem 9.7.4]). Next we add that the proof of Theorem 1.1 can in a straightforward way be extended to the case where $\varphi$ has at most polynomially growing derivatives. It is, however, not clear to us how to treat the case where $F$ and $B$ are globally Lipschitz continuous but with the first four derivatives growing polynomially. Furthermore, we emphasize that Theorem 1.1 solves the weak convergence problem emerged from Debussche's article (see (2.5) and Remark 2.3 in Debussche [19]) merely in the case of spatial spectral Galerkin approximations instead of other more complicated numerical approximations for the SEE (11). The method of proof of our weak convergence results, however, can be extended to a number of other kind of spatial and temporal numerical approximations for SEEs of the form (1). In particular, in our proceeding article [29] we extend the method of proof developed here to 
establish essentially sharp weak convergence rates for different types of temporal numerical approximations (such as exponential Euler \{see [29, Subsection 1.5.1]\} and linear-implicit Euler $\{$ see [29, Subsection 1.5.2]\} approximations for SPDEs) for SPDEs with possibly non-constant diffusion coefficients without neither assuming (2) nor that $B$ maps from $H$ to $L(H)$. Next we point out that the proof in Debussche's article [19] as well as many other proofs in the above mentioned weak convergence articles use Malliavin calculus. Our method of proof does not use Malliavin calculus but uses - in some sense - merely elementary arguments as well as the mild Itô formula in Da Prato et al. 14.

The paper is organized as follows. In Section 1.1 below we give a rough sketch of the proof of Theorem 1.1 without technical details. However, the main ideas that we use to obtain an essentially sharp rate of convergence are highlighted in Section 1.1 below. In Section 1.2 we illustrate Theorem 1.1 and Corollary 6.1, respectively, by two simple examples. Sections 1.3 and 1.4 present the notation and the framework used in this paper. Section 2 studies weak convergence rates for the spectral Galerkin projections $P_{N}\left(X_{T}\right), N \in \mathbb{N}$, associated to the solution process $X_{t}, t \in[0, T]$, of the SEE (11). The result of this section is then used in Section 3 to obtain the weak convergence of the Galerkin approximation (3) to the solution of (11) in the case where the drift operator $F$, the diffusion operator $B$, and the initial condition are mollified in an appropriate sense. This provides a less general version of Theorem 1.1. Section 4 is devoted to the proof of an elementary strong convergence result. In Section 5 the weak convergence result from Section 3 and the elementary strong convergence result from Section 4 are used to establish weak convergence (see Corollary [5.3) for general drift and diffusion operators. Section 6 specializes the weak convergence result from Section 5 to the framework of this introductory section. Finally, in Section 7 we consider the case $F=0$ and provide examples of constant (additive noise) functions $B$ which show that the weak convergence rate established in Theorem 1.1 can essentially not be improved.

\subsection{Sketch of the proof of the main weak convergence result}

In the following we give a brief sketch of our method of proof of Theorem 1.1 and Corollary 6.1. respectively, in the case where $\xi \in H_{\iota+2}$ (the case where $\xi \in H_{\iota}$ then follows from a standard mollification procedure; see (239) in the proof of Proposition 5.2 in Section 5 for details). In our weak convergence proof we intend to work (as it is often the case in the case of weak convergence for S(P)DEs; see, e.g., Rößler [43] and Debussche [19]) with the Kolmogorov backward equation associated to (1). In the case of an SEE with a general nonlinear diffusion coefficient it is, however, not clear whether the solutions of the SEE (1) also provide strong solutions of the Kolmogorov backward equation associated to (11); cf. [1, item (iv) of Theorem 1.1], [26, Corollary 1.2], and [13, pages 249-251]. We therefore work with suitable mollified versions of (11) and (3). More formally, for every $\kappa \in(0, \infty)$ let $F_{\kappa}: H_{\iota} \rightarrow H_{\iota+2}$ and $B_{\kappa}: H_{\iota} \rightarrow H S\left(U, H_{\iota+2}\right)$ be the functions which satisfy for all $x \in H_{\iota}$ that $F_{\kappa}(x)=e^{\kappa A} F(x)$ and $B_{\kappa}(x)=e^{\kappa A} B(x)$. For every $\kappa \in(0, \infty), x \in H_{\iota}$ let $\hat{X}^{x, \kappa}:[0, T] \times \Omega \rightarrow H_{\iota}$ be a continuous mild solution of the SEE

$$
d \hat{X}_{t}^{x, \kappa}=\left[A \hat{X}_{t}^{x, \kappa}+F_{\kappa}\left(\hat{X}_{t}^{x, \kappa}\right)\right] d t+B_{\kappa}\left(\hat{X}_{t}^{x, \kappa}\right) d W_{t}, \quad t \in[0, T], \quad \hat{X}_{0}^{x, \kappa}=x .
$$

For every $\kappa \in(0, \infty)$ let $u_{\kappa}:[0, T] \times H_{\iota} \rightarrow \mathbb{R}$ be the function which satisfies for all $(t, x) \in[0, T] \times H_{\iota}$ that $u_{\kappa}(t, x)=\mathbb{E}\left[\varphi\left(\hat{X}_{T-t}^{x, \kappa}\right)\right]$. In particular, notice that for all $\kappa \in(0, \infty)$ and all nonrandom $x \in H_{\iota}$ it holds that $u_{\kappa}(T, x)=\varphi(x)$. Then, for every $\kappa \in(0, \infty)$, $N \in \mathbb{N}$ let $X^{N, \kappa}:[0, T] \times \Omega \rightarrow H_{\iota}$ be a continuous mild solution of the SEE

$$
d X_{t}^{N, \kappa}=\left[P_{N} A X_{t}^{N, \kappa}+P_{N} F_{\kappa}\left(X_{t}^{N, \kappa}\right)\right] d t+P_{N} B_{\kappa}\left(X_{t}^{N, \kappa}\right) d W_{t}, \quad t \in[0, T], \quad X_{0}^{N, \kappa}=P_{N}(\xi) .
$$


The first key idea in our proof is then to bring certain modified versions of the SEEs (3) and (8) respectively into play to analyze the weak approximation errors $\mid \mathbb{E}\left[\varphi\left(\hat{X}_{T}^{\xi, \kappa}\right)\right]-$ $\mathbb{E}\left[\varphi\left(X_{T}^{N, \kappa}\right)\right] \mid$ for $N \in \mathbb{N}, \kappa \in(0, \infty)$. More specifically, for every $\kappa \in(0, \infty), N \in \mathbb{N}$ let $Y^{N, \kappa}:[0, T] \times \Omega \rightarrow H_{\iota+2}$ be a continuous mild solution of the SEE

$$
d Y_{t}^{N, \kappa}=\left[A Y_{t}^{N, \kappa}+F_{\kappa}\left(P_{N}\left(Y_{t}^{N, \kappa}\right)\right)\right] d t+B_{\kappa}\left(P_{N}\left(Y_{t}^{N, \kappa}\right)\right) d W_{t}, \quad t \in[0, T], \quad Y_{0}^{N, \kappa}=\xi .
$$

It is crucial in (9) that $P_{N}(\cdot)$ appears inside the arguments of $F_{\kappa}$ and $B_{\kappa}$ instead of in front of $F_{\kappa}$ and $B_{\kappa}$ as in (8) (and (3) ). Moreover, notice the projection $P_{N}\left(Y_{t}^{N, \kappa}\right)=X_{t}^{N, \kappa}$ $\mathbb{P}$-a.s. for all $N \in \mathbb{N}, \kappa \in(0, \infty), t \in[0, T]$. To estimate the weak approximation errors $\left|\mathbb{E}\left[\varphi\left(\hat{X}_{T}^{\xi, \kappa}\right)\right]-\mathbb{E}\left[\varphi\left(X_{T}^{N, \kappa}\right)\right]\right|$ for $N \in \mathbb{N}, \kappa \in(0, \infty)$ we then apply the triangle inequality to obtain that for all $\kappa \in(0, \infty), N \in \mathbb{N}$ it holds that

$$
\begin{aligned}
\mid \mathbb{E} & {\left[\varphi\left(\hat{X}_{T}^{\xi, \kappa}\right)\right]-\mathbb{E}\left[\varphi\left(X_{T}^{N, \kappa}\right)\right] \mid } \\
& \leq\left|\mathbb{E}\left[\varphi\left(\hat{X}_{T}^{\xi, \kappa}\right)\right]-\mathbb{E}\left[\varphi\left(Y_{T}^{N, \kappa}\right)\right]\right|+\left|\mathbb{E}\left[\varphi\left(Y_{T}^{N, \kappa}\right)\right]-\mathbb{E}\left[\varphi\left(X_{T}^{N, \kappa}\right)\right]\right| \\
& =\left|u_{\kappa}(0, \xi)-\mathbb{E}\left[u_{\kappa}\left(T, Y_{T}^{N, \kappa}\right)\right]\right|+\left|\mathbb{E}\left[\varphi\left(Y_{T}^{N, \kappa}\right)\right]-\mathbb{E}\left[\varphi\left(P_{N}\left(Y_{T}^{N, \kappa}\right)\right)\right]\right| \\
& =\left|\mathbb{E}\left[u_{\kappa}\left(T, Y_{T}^{N, \kappa}\right)-u_{\kappa}\left(0, Y_{0}^{N, \kappa}\right)\right]\right|+\left|\mathbb{E}\left[\varphi\left(Y_{T}^{N, \kappa}\right)\right]-\mathbb{E}\left[\varphi\left(P_{N}\left(Y_{T}^{N, \kappa}\right)\right)\right]\right| .
\end{aligned}
$$

Roughly speaking, the processes $Y^{N, \kappa}, N \in \mathbb{N}, \kappa \in(0, \infty)$, are chosen in such a way so that it is not so difficult anymore to estimate $\left|\mathbb{E}\left[u_{\kappa}\left(T, Y_{T}^{N, \kappa}\right)-u_{\kappa}\left(0, Y_{0}^{N, \kappa}\right)\right]\right|$ and $\left|\mathbb{E}\left[\varphi\left(Y_{T}^{N, \kappa}\right)\right]-\mathbb{E}\left[\varphi\left(P_{N}\left(Y_{T}^{N, \kappa}\right)\right)\right]\right|$ on the right hand side of (10). More formally, to estimate the term $\left|\mathbb{E}\left[\varphi\left(Y_{T}^{N, \kappa}\right)\right]-\mathbb{E}\left[\varphi\left(P_{N}\left(Y_{T}^{N, \kappa}\right)\right)\right]\right|$ on the right hand side of (10) (see Section 2 and Lemma 3.5 in Section 3) we apply the mild Itô formula in Corollary 2 in Da Prato et al. [14] to $\mathbb{E}\left[\varphi\left(Y_{t}^{N, \kappa}\right)\right], t \in[0, T]$, and to $\mathbb{E}\left[\varphi\left(P_{N}\left(Y_{T}^{N, \kappa}\right)\right)\right], t \in[0, T]$, and then estimate the difference of the resulting terms in a straightforward way (see the proof of Proposition 2.1 in Section 2 below for details). This allows us to prove (see Proposition 2.1 below) that there exist real numbers $C_{\varepsilon}^{(1)} \in[0, \infty), \varepsilon \in(0, \infty)$, such that for all $\varepsilon, \kappa \in(0, \infty), N \in \mathbb{N}$ it holds that

$$
\left|\mathbb{E}\left[\varphi\left(Y_{T}^{N, \kappa}\right)\right]-\mathbb{E}\left[\varphi\left(X_{T}^{N, \kappa}\right)\right]\right|=\left|\mathbb{E}\left[\varphi\left(Y_{T}^{N, \kappa}\right)\right]-\mathbb{E}\left[\varphi\left(P_{N}\left(Y_{T}^{N, \kappa}\right)\right)\right]\right| \leq C_{\varepsilon}^{(1)}\left(\lambda_{N}\right)^{-(1-\gamma-\varepsilon)} .
$$

To estimate the term $\left|\mathbb{E}\left[u_{\kappa}\left(T, Y_{T}^{N, \kappa}\right)-u_{\kappa}\left(0, Y_{0}^{N, \kappa}\right)\right]\right|$ on the right hand side of (10) we apply the standard Itô formula to the stochastic processes $\left(u_{\kappa}\left(t, Y_{t}^{N, \kappa}\right)\right)_{t \in[0, T]}, \kappa \in(0, \infty)$, and use the fact that the functions $u_{\kappa}, \kappa \in(0, \infty)$, solve the Kolmogorov backward equation associated to (7) to obtain that for all $\kappa \in(0, \infty), N \in \mathbb{N}$ it holds that

$$
\begin{aligned}
& \left|\mathbb{E}\left[u_{\kappa}\left(T, Y_{T}^{N, \kappa}\right)-u_{\kappa}\left(0, Y_{0}^{N, \kappa}\right)\right]\right| \leq \int_{0}^{T}\left|\mathbb{E}\left[\left(\frac{\partial}{\partial x} u_{\kappa}\right)\left(s, Y_{s}^{N, \kappa}\right)\left(F_{\kappa}\left(P_{N}\left(Y_{s}^{N, \kappa}\right)\right)-F_{\kappa}\left(Y_{s}^{N, \kappa}\right)\right)\right]\right| d s \\
& +\sum_{b \in \mathbb{U}} \int_{0}^{T} \frac{\left|\mathbb{E}\left[\left(\frac{\partial^{2}}{\partial x^{2}} u_{\kappa}\right)\left(s, Y_{s}^{N, \kappa}\right)\left(\left[B_{\kappa}\left(P_{N}\left(Y_{s}^{N, \kappa}\right)\right)+B_{\kappa}\left(Y_{s}^{N, \kappa}\right)\right] b,\left[B_{\kappa}\left(P_{N}\left(Y_{s}^{N, \kappa}\right)\right)-B_{\kappa}\left(Y_{s}^{N, \kappa}\right)\right] b\right)\right]\right|}{2} d s
\end{aligned}
$$

where $\mathbb{U} \subseteq U$ is an arbitrary orthonormal basis of $U$; cf. (174) in Section 3 below. The next key idea in our weak convergence proof is then to again apply the mild Itô formula (see Da Prato et al. [14]) to the terms appearing on the right hand side of (12). After applying the mild Itô formula, the resulting terms can be estimated in a straightforward way by using the estimates for the functions $u_{\kappa}, \kappa \in(0, T]$, from Andersson et al. [1]. This allows us (cf. (162) in Lemma 3.7 and (242)-(243) in the proof of Proposition 5.2) to prove that for all $\varepsilon \in(0, \infty)$ there exists a real number $C_{\varepsilon}^{(2)} \in[0, \infty)$ such that for all $\kappa \in(0, T], N \in \mathbb{N}$ it holds that

$$
\left|\mathbb{E}\left[\varphi\left(\hat{X}_{T}^{\xi, \kappa}\right)\right]-\mathbb{E}\left[\varphi\left(Y_{T}^{N, \kappa}\right)\right]\right|=\left|\mathbb{E}\left[u_{\kappa}\left(T, Y_{T}^{N, \kappa}\right)-u_{\kappa}\left(0, Y_{0}^{N, \kappa}\right)\right]\right| \leq \frac{C_{\varepsilon}^{(2)}}{\kappa^{\varepsilon}\left(\lambda_{N}\right)^{(1-\gamma-\varepsilon)}} .
$$


Putting (13) and (11) into (10) then proves that for all $\varepsilon \in(0, \infty), \kappa \in(0, T], N \in \mathbb{N}$ it holds that

$$
\left|\mathbb{E}\left[\varphi\left(\hat{X}_{T}^{\xi, \kappa}\right)\right]-\mathbb{E}\left[\varphi\left(X_{T}^{N, \kappa}\right)\right]\right| \leq C_{\varepsilon}^{(2)} \kappa^{-\varepsilon}\left(\lambda_{N}\right)^{-(1-\gamma-\varepsilon)}+C_{\varepsilon}^{(1)}\left(\lambda_{N}\right)^{-(1-\gamma-\varepsilon)} .
$$

Estimates (13) and (14) illustrate that we cannot simply let the mollifying parameter $\kappa$ tend to 0 because the right hand side of (14) diverges as $\kappa$ tends to 0 . The last key idea in our proof is then to make use of the following - somehow nonstandard - mollification procedure to overcome this problem. For this mollification procedure we first use wellknown strong convergence analysis to prove (cf. Proposition 4.1 and Corollary 4.2 in Section 4) that for all $\varepsilon \in(0, \infty)$ there exists a real number $C_{\varepsilon}^{(3)} \in[0, \infty)$ such that for all $\kappa \in(0, T], N \in \mathbb{N}$ it holds that

$$
\left|\mathbb{E}\left[\varphi\left(X_{T}\right)\right]-\mathbb{E}\left[\varphi\left(\hat{X}_{T}^{\xi, \kappa}\right)\right]\right|+\left|\mathbb{E}\left[\varphi\left(X_{T}^{N}\right)\right]-\mathbb{E}\left[\varphi\left(X_{T}^{N, \kappa}\right)\right]\right| \leq C_{\varepsilon}^{(3)} \kappa^{\left(\frac{1-\gamma}{2}-\varepsilon\right)} .
$$

Combining (15) with (14) then shows that for all $\varepsilon \in(0, \infty), \kappa \in(0, T], N \in \mathbb{N}$ it holds that

$$
\left|\mathbb{E}\left[\varphi\left(X_{T}\right)\right]-\mathbb{E}\left[\varphi\left(X_{T}^{N}\right)\right]\right| \leq \frac{C_{\varepsilon}^{(1)}}{\left(\lambda_{N}\right)^{(1-\gamma-\varepsilon)}}+\frac{C_{\varepsilon}^{(2)}}{\kappa^{\varepsilon}\left(\lambda_{N}\right)^{(1-\gamma-\varepsilon)}}+C_{\varepsilon}^{(3)} \kappa^{\left(\frac{1-\gamma}{2}-\varepsilon\right)} .
$$

As the left hand side of (16) is independent of $\kappa \in(0, T]$, we can minimize the right hand side of (16) over $\kappa \in(0, T]$ (instead of letting $\kappa$ tend to 0$)$ and this will allow us to complete the proof of Theorem 1.1) see (244) and (246) in the proof of Proposition 5.2 in Section 5 below for details.

\subsection{Examples}

In this section we illustrate Theorem 1.1 and Corollary 6.1, respectively, by two simple examples. In Subsection 1.2.1 we apply Theorem 1.1 to the continuous version of the onedimensional parabolic Anderson model and in Subsection 1.2.2 we apply Theorem 1.1 to a Cahn-Hilliard-Cook type equation.

\subsubsection{Parabolic Anderson model and nonlinear heat-type SPDEs}

Let $H=L^{2}((0,1) ; \mathbb{R})$ be the $\mathbb{R}$-Hilbert space of equivalence classes of Lebesgue-Borel square integrable functions from $(0,1)$ to $\mathbb{R}$, let $T, \kappa, \delta, \nu \in(0, \infty), \xi \in H$, let $(\Omega, \mathcal{F}, \mathbb{P}$, $\left.\left(\mathcal{F}_{t}\right)_{t \in[0, T]}\right)$ be a stochastic basis, let $\left(W_{t}\right)_{t \in[0, T]}$ be an $\operatorname{Id}_{H}$-cylindrical $\left(\Omega, \mathcal{F}, \mathbb{P},\left(\mathcal{F}_{t}\right)_{t \in[0, T]}\right)$ Wiener process, let $e_{n} \in H, n \in \mathbb{N}$, be the orthonormal basis of $H$ which satisfies for all $n \in \mathbb{N}$ that $e_{n}=\sqrt{2} \sin (n \pi(\cdot))$, let $A: D(A) \subseteq H \rightarrow H$ be the linear operator which satisfies $D(A)=\left\{v \in H: \sum_{n=1}^{\infty} n^{4}\left|\left\langle e_{n}, v\right\rangle_{H}\right|^{2}<\infty\right\}$ and $\forall v \in D(A): A v=$ $\sum_{n=1}^{\infty}-\nu n^{2} \pi^{2}\left\langle e_{n}, v\right\rangle_{H} e_{n}$, let $\left(H_{r},\langle\cdot, \cdot\rangle_{H_{r}},\|\cdot\|_{H_{r}}\right), r \in \mathbb{R}$, be a family of interpolation spaces associated to $-A$, let $\left(P_{N}\right)_{N \in \mathbb{N}} \subseteq L\left(H_{-1}\right)$ satisfy for all $N \in \mathbb{N}, v \in H$ that $P_{N}(v)=\sum_{n=1}^{N}\left\langle e_{n}, v\right\rangle_{H} e_{n}$, let $\psi: H \rightarrow \bar{H}$ be a four times continuously Fréchet differentiable function with globally bounded derivatives, and let $B: H \rightarrow H S\left(H, H_{-1 / 4-\delta}\right)$ be the function which satisfies for all $v \in H$ and all uniformly continuous functions $u:(0,1) \rightarrow \mathbb{R}$ that $B(v) u=\psi(v) \cdot u$. The above assumptions ensure the existence of $\left(\mathcal{F}_{t}\right)_{t \in[0, T]}$-adapted continuous stochastic processes $X:[0, T] \times \Omega \rightarrow H$ and $X^{N}:[0, T] \times \Omega \rightarrow P_{N}(H), N \in \mathbb{N}$, which satisfy that for all $N \in \mathbb{N}, t \in[0, T]$ it holds $\mathbb{P}$-a.s. that

$$
X_{t}=e^{A t} \xi+\int_{0}^{t} e^{A(t-s)} B\left(X_{s}\right) d W_{s}
$$


and $X_{t}^{N}=e^{A t} P_{N}(\xi)+\int_{0}^{t} e^{A(t-s)} P_{N} B\left(X_{s}^{N}\right) d W_{s}$. In the case where $\forall v \in H: \psi(v)=$ $\left[1+\|v\|_{H}^{2}\right]^{-1} v$ the stochastic process $X$ is a mild solution process of

$$
d X_{t}(x)=\nu \frac{\partial^{2}}{\partial x^{2}} X_{t}(x) d t+\frac{X_{t}(x)}{1+\int_{0}^{1}\left|X_{t}(y)\right|^{2} d y} d W_{t}(x)
$$

with $X_{t}(0)=X_{t}(1)=0$ and $X_{0}(x)=\xi(x)$ for $x \in(0,1), t \in[0, T]$ and the stochastic processes $X^{N}, N \in \mathbb{N}$, are spatial spectral Galerkin approximations of (18). In the case where $\forall v \in H: \psi(v)=\kappa \cdot v$ the stochastic process $X$ is a mild solution process of the continuous version of the one-dimensional parabolic Anderson model

$$
d X_{t}(x)=\nu \frac{\partial^{2}}{\partial x^{2}} X_{t}(x) d t+\kappa X_{t}(x) d W_{t}(x)
$$

with $X_{t}(0)=X_{t}(1)=0$ and $X_{0}(x)=\xi(x)$ for $x \in(0,1), t \in[0, T]$ (cf., e.g., Carmona \& Molchanov [10]) and the stochastic processes $X^{N}, N \in \mathbb{N}$, are spatial spectral Galerkin approximations of (19). Theorem 1.1 and Corollary 6.1, respectively, apply here with $\gamma=$ $1 / 2$, that is, Theorem 1.1 and Corollary 6.1, respectively, ensure that for all $\varphi \in C_{b}^{4}(H, \mathbb{R})$, $\varepsilon \in(0, \infty)$ it holds that there exists a real number $C \in \mathbb{R}$ such that for all $N \in \mathbb{N}$ it holds that

$$
\left|\mathbb{E}\left[\varphi\left(X_{T}\right)\right]-\mathbb{E}\left[\varphi\left(X_{T}^{N}\right)\right]\right| \leq C \cdot N^{-(1-\varepsilon)} .
$$

Theorem 1.1 and Corollary 6.1, respectively, thus demonstrate that the spatial spectral Galerkin approximations $X^{N}, N \in \mathbb{N}$, of (17), (18), and (19), respectively, converge with rate $1-\varepsilon$ to the stochastic process $X$ of (17), (18), and (19). To the best of our knowledge, Theorem 1.1 and Corollary 6.1, respectively, are the first results in the scientific literature which establish essentially sharp weak convergence rates for numerical approximations of (18) and (19), respectively.

\subsubsection{A Cahn-Hilliard-Cook type equation}

Let $H=L^{2}((0,1) ; \mathbb{R})$ be the $\mathbb{R}$-Hilbert space of equivalence classes of Lebesgue-Borel square integrable functions from $(0,1)$ to $\mathbb{R}$, let $T, \kappa, \delta \in(0, \infty), \xi \in H$, let $(\Omega, \mathcal{F}, \mathbb{P}$, $\left.\left(\mathcal{F}_{t}\right)_{t \in[0, T]}\right)$ be a stochastic basis, let $\left(W_{t}\right)_{t \in[0, T]}$ be an $\operatorname{Id}_{H}$-cylindrical $\left(\Omega, \mathcal{F}, \mathbb{P},\left(\mathcal{F}_{t}\right)_{t \in[0, T]}\right)$ Wiener process, let $e_{n} \in H, n \in \mathbb{N}_{0}$, be the orthonormal basis of $H$ which satisfies for all $n \in \mathbb{N}$ that $e_{0}=1$ and $e_{n}=\sqrt{2} \cos (n \pi(\cdot))$, let $A: D(A) \subseteq H \rightarrow H$ be the linear operator which satisfies $D(A)=\left\{v \in H: \sum_{n \in \mathbb{N}} n^{8}\left|\left\langle e_{n}, v\right\rangle_{H}\right|^{2}<\infty\right\}$ and $\forall v \in$ $D(A): A v=\sum_{n=0}^{\infty}\left(n^{2} \pi^{2}-n^{4} \pi^{4}-1\right)\left\langle e_{n}, v\right\rangle_{H} e_{n}$, let $\left(H_{r},\langle\cdot, \cdot\rangle_{H_{r}},\|\cdot\|_{H_{r}}\right), r \in \mathbb{R}$, be a family of interpolation spaces associated to $-A$, let $\left(P_{N}\right)_{N \in \mathbb{N}} \subseteq L\left(H_{-1}\right)$ satisfy for all $N \in \mathbb{N}, v \in H$ that $P_{N}(v)=\sum_{n=0}^{N}\left\langle e_{n}, v\right\rangle_{H} e_{n}$, and let $F: H \rightarrow H_{-1 / 4-2 \delta}$ and $B: H \rightarrow$ $H S\left(H, H_{-1 / 8-\delta}\right)$ satisfy for all $v \in H$ and all uniformly continuous functions $u:(0,1) \rightarrow \mathbb{R}$ that $F(v)=v$ and $B(v) u=\kappa \cdot v \cdot u$.

The above assumptions ensure the existence of $\left(\mathcal{F}_{t}\right)_{t \in[0, T]}$-adapted continuous stochastic processes $X:[0, T] \times \Omega \rightarrow H$ and $X^{N}:[0, T] \times \Omega \rightarrow P_{N}(H), N \in \mathbb{N}$, which satisfy that for all $N \in \mathbb{N}, t \in[0, T]$ it holds $\mathbb{P}$-a.s. that

$$
X_{t}=e^{A t} \xi+\int_{0}^{t} e^{A(t-s)} F\left(X_{s}\right) d s+\int_{0}^{t} e^{A(t-s)} B\left(X_{s}\right) d W_{s}
$$

and $X_{t}^{N}=e^{A t} P_{N}(\xi)+\int_{0}^{t} e^{A(t-s)} P_{N} F\left(X_{s}^{N}\right) d s+\int_{0}^{t} e^{A(t-s)} P_{N} B\left(X_{s}^{N}\right) d W_{s}$. The stochastic process $X$ is thus a solution process of the Cahn-Hilliard-Cook type equation

$$
d X_{t}(x)=\left[-\frac{\partial^{4}}{\partial x^{4}} X_{t}(x)-\frac{\partial^{2}}{\partial x^{2}} X_{t}(x)\right] d t+\kappa X_{t}(x) d W_{t}(x)
$$


with $X_{t}^{\prime}(0)=X_{t}^{\prime}(1)=X_{t}^{(3)}(0)=X_{t}^{(3)}(1)=0$ and $X_{0}(x)=\xi(x)$ for $x \in(0,1), t \in[0, T]$ and the stochastic processes $X^{N}, N \in \mathbb{N}$, are spatial spectral Galerkin approximations of (22). Theorem 1.1 and Corollary 6.1. respectively, apply here with $\gamma=1 / 4$, that is, Theorem 1.1 and Corollary 6.1, respectively, ensure that for all $\varphi \in C_{b}^{4}(H, \mathbb{R}), \varepsilon \in(0, \infty)$ it holds that there exists a real number $C \in \mathbb{R}$ such that for all $N \in \mathbb{N}$ it holds that

$$
\left|\mathbb{E}\left[\varphi\left(X_{T}\right)\right]-\mathbb{E}\left[\varphi\left(X_{T}^{N}\right)\right]\right| \leq C \cdot N^{-(3-\varepsilon)} .
$$

Theorem 1.1 and Corollary 6.1, respectively, thus demonstrate that the spatial spectral Galerkin approximations $X^{N}, N \in \mathbb{N}$, of (22) converge with rate $3-\varepsilon$ to the solution process $X$ of (22). To the best of our knowledge, Theorem 1.1 and Corollary 6.1, respectively, are the first results in the scientific literature which establish essentially sharp weak convergence rates for numerical approximations of (22).

\subsection{Notation}

Throughout this article the following notation is used. For every set $S$ we denote by $\operatorname{Id}_{S}: S \rightarrow S$ the identity mapping on $S$. For every set $S$ we denote by $\mathcal{P}(S)$ the power set of $S$. We denote by $\mathcal{E}_{r}:[0, \infty) \rightarrow[0, \infty), r \in(0, \infty)$, the functions which satisfy for all $r \in(0, \infty), x \in[0, \infty)$ that $\mathcal{E}_{r}(x)=\left[\sum_{n=0}^{\infty} \frac{x^{2 n} \Gamma(r)^{n}}{\Gamma(n r+1)}\right]^{1 / 2}$ (generalized exponential function; cf., e.g., Exercise 3 in Chapter 7 in Henry [27], (1.0.1) in Chapter 1 in Gorenflo et al. [23], and (16) in Andersson et al. [2]). For all normed $\mathbb{R}$-vector spaces $\left(E_{1},\|\cdot\|_{E_{1}}\right)$ and $\left(E_{2},\|\cdot\|_{E_{2}}\right)$ and every nonnegative integer $k \in \mathbb{N}_{0}$ we denote by $|\cdot|_{\operatorname{Lip}^{k}\left(E_{1}, E_{2}\right)},\|\cdot\|_{\operatorname{Lip}^{k}\left(E_{1}, E_{2}\right)}$ : $C^{k}\left(E_{1}, E_{2}\right) \rightarrow[0, \infty]$ the functions which satisfy for all $f \in C^{k}\left(E_{1}, E_{2}\right)$ that

$$
|f|_{\operatorname{Lip}^{k}\left(E_{1}, E_{2}\right)}= \begin{cases}\sup _{x, y \in E_{1}} \frac{\|f(x)-f(y)\|_{E_{2}}}{\|x-y\|_{E_{1}}} & : k=0 \\ \sup _{\substack{x, y \in E_{1} \\ x \neq y}} \frac{\left\|f^{(k)}(x)-f^{(k)}(y)\right\|_{L^{(k)}\left(E_{1}, E_{2}\right)}}{\|x-y\|_{E_{1}}} & : k \in \mathbb{N}\end{cases}
$$

and $\|f\|_{\operatorname{Lip}^{k}\left(E_{1}, E_{2}\right)}=\|f(0)\|_{E_{2}}+\sum_{l=0}^{k}|f|_{\operatorname{Lip}^{l}\left(E_{1}, E_{2}\right)}$ and we denote by $\operatorname{Lip}^{k}\left(E_{1}, E_{2}\right)$ the set given by $\operatorname{Lip}^{k}\left(E_{1}, E_{2}\right)=\left\{f \in C^{k}\left(E_{1}, E_{2}\right):\|f\|_{\operatorname{Lip}^{k}\left(E_{1}, E_{2}\right)}<\infty\right\}$. For all normed $\mathbb{R}$-vector spaces $\left(E_{1},\|\cdot\|_{E_{1}}\right)$ and $\left(E_{2},\|\cdot\|_{E_{2}}\right)$ and every natural number $k \in \mathbb{N}$ we denote by $|\cdot|_{C_{b}^{k}\left(E_{1}, E_{2}\right)},\|\cdot\|_{C_{b}^{k}\left(E_{1}, E_{2}\right)}: C^{k}\left(E_{1}, E_{2}\right) \rightarrow[0, \infty]$ the functions which satisfy for all $f \in C^{k}\left(E_{1}, E_{2}\right)$ that $|f|_{C_{b}^{k}\left(E_{1}, E_{2}\right)}=\sup _{x \in E_{1}}\left\|f^{(k)}(x)\right\|_{L^{(k)}\left(E_{1}, E_{2}\right)}$ and $\|f\|_{C_{b}^{k}\left(E_{1}, E_{2}\right)}=$ $\|f(0)\|_{E_{2}}+\sum_{l=1}^{k}|f|_{C_{b}^{l}\left(E_{1}, E_{2}\right)}$ and we denote by $C_{b}^{k}\left(E_{1}, E_{2}\right)$ the set given by $C_{b}^{k}\left(E_{1}, E_{2}\right)=$ $\left\{f \in C^{k}\left(E_{1}, E_{2}\right):\|f\|_{C_{b}^{k}\left(E_{1}, E_{2}\right)}<\infty\right\}$.

\subsection{Setting}

Throughout this article the following setting is frequently used. Consider the notation in Section 1.3, let $\left(H,\langle\cdot, \cdot\rangle_{H},\|\cdot\|_{H}\right)$ and $\left(U,\langle\cdot, \cdot\rangle_{U},\|\cdot\|_{U}\right)$ be separable $\mathbb{R}$-Hilbert spaces, let $T \in(0, \infty)$, let $\left(\Omega, \mathcal{F}, \mathbb{P},\left(\mathcal{F}_{t}\right)_{t \in[0, T]}\right)$ be a stochastic basis, let $\left(W_{t}\right)_{t \in[0, T]}$ be an $\operatorname{Id}_{U^{-}}$ cylindrical $\left(\Omega, \mathcal{F}, \mathbb{P},\left(\mathcal{F}_{t}\right)_{t \in[0, T]}\right)$-Wiener process, let $\mathbb{H} \subseteq H$ be a nonempty orthonormal basis, let $\lambda: \mathbb{H} \rightarrow \mathbb{R}$ be a function satisfying $\sup _{b \in \mathbb{H}} \lambda_{b}<0$, let $A: D(A) \subseteq H \rightarrow H$ be a linear operator which satisfies $D(A)=\left\{v \in H: \sum_{b \in \mathbb{H}}\left|\lambda_{b}\langle b, v\rangle_{H}\right|^{2}<\infty\right\}$ and $\forall v \in$ $D(A): A v=\sum_{b \in \mathbb{H}} \lambda_{b}\langle b, v\rangle_{H} b$, let $\left(H_{r},\langle\cdot, \cdot\rangle_{H_{r}},\|\cdot\|_{H_{r}}\right), r \in \mathbb{R}$, be a family of interpolation spaces associated to $-A$, and let $\left(P_{I}\right)_{I \in \mathcal{P}(\mathbb{H})} \subseteq L\left(H_{-1}\right)$ satisfy for all $v \in H, I \in \mathcal{P}(\mathbb{H})$ that $P_{I}(v)=\sum_{b \in I}\langle b, v\rangle_{H} b$. 


\subsection{Auxiliary lemmas}

Throughout this article we frequently use the following well-known lemmas.

Lemma 1.2. Assume the setting in Section 1.4. Then it holds for all $r \in[0,1]$ that $\sup _{t \in[0, \infty)}\left\|(-t A)^{r} e^{A t}\right\|_{L(H)} \leq \sup _{x \in(0, \infty)}\left[\frac{x^{r}}{e^{x}}\right] \leq\left[\frac{r}{e}\right]^{r} \leq 1$.

Lemma 1.3 (See, e.g., Lemma 2.2 in Andersson et al. [2]). Let $\left(V_{k},\|\cdot\|_{V_{k}}\right), k \in\{0,1\}$, be separable $\mathbb{R}$-Banach spaces with $V_{1} \subseteq V_{0}$ continuously. Then

$$
\mathcal{B}\left(V_{1}\right)=\left\{B \in \mathcal{P}\left(V_{1}\right):\left(\exists A \in \mathcal{B}\left(V_{0}\right): B=A \cap V_{1}\right)\right\} \subseteq \mathcal{B}\left(V_{0}\right) .
$$

\section{Weak convergence for Galerkin projections of SEEs}

In this section we establish weak convergence rates for Galerkin projections of SEEs (see Proposition 2.1 below). More specifically, in the framework of Section 1.4 we establish in Proposition 2.1 below an explicit upper bound for the weak approximation error

$$
\left|\mathbb{E}\left[\varphi\left(X_{T}\right)\right]-\mathbb{E}\left[\varphi\left(P_{I}\left(X_{T}\right)\right)\right]\right|,
$$

where $I \subseteq \mathbb{H}$ is a set, where $\varphi: H \rightarrow \mathbb{R}$ is a twice continuously Fréchet differentiable function with globally bounded and globally Lipschitz continuous derivatives, and where $X:[0, T] \times \Omega \rightarrow H$ is a suitable mild solution process of the SEE (27). In this section the nonlinearities in the SEE (27) are not mollified and may take values in appropriate negative interpolation spaces. Proposition [2.1, in particular, proves inequality (11) in Section 1.1. In Corollary [3.8 in Section 3 below we will use Proposition 2.1 to establish weak convergence rates for Galerkin approximations of SEEs with mollified nonlinearities. In particular, in Section 3 we establish upper error bounds for the first summand on the right hand side of (10) (see Lemma 3.7 in Subsection 3.3 below) and we use these upper error bounds together with Proposition 2.1 in this section to obtain upper error bounds for the left hand side of (10). Proposition 2.1 is a slightly modified version of Corollary 8 in Da Prato et al. [14].

\subsection{Setting}

Assume the setting in Section 1.4 and let $\vartheta \in[0,1), F \in \operatorname{Lip}^{0}\left(H, H_{-\vartheta}\right), B \in \operatorname{Lip}^{0}(H, H S(U$, $\left.\left.H_{-\vartheta / 2}\right)\right), \varphi \in \operatorname{Lip}^{2}(H, \mathbb{R}), \xi \in L^{3}\left(\left.\mathbb{P}\right|_{\mathcal{F}_{0}} ; H\right)$.

The above assumptions ensure that there exists an up-to-modifications unique $\left(\mathcal{F}_{t}\right)_{t \in[0, T]^{-}}$ predictable stochastic process $X:[0, T] \times \Omega \rightarrow H$ which satisfies $\sup _{t \in[0, T]}\left\|X_{t}\right\|_{L^{3}(\mathbb{P} ; H)}<\infty$ and which satisfies that for all $t \in[0, T]$ it holds $\mathbb{P}$-a.s. that

$$
X_{t}=e^{A t} \xi+\int_{0}^{t} e^{A(t-s)} F\left(X_{s}\right) d s+\int_{0}^{t} e^{A(t-s)} B\left(X_{s}\right) d W_{s}
$$

\subsection{A weak convergence result}

Proposition 2.1. Assume the setting in Section 2.1 and let $\rho \in[0,1-\vartheta), I \in \mathcal{P}(\mathbb{H})$. Then

$$
\begin{aligned}
& \left|\mathbb{E}\left[\varphi\left(X_{T}\right)\right]-\mathbb{E}\left[\varphi\left(P_{I}\left(X_{T}\right)\right)\right]\right| \leq\|\varphi\|_{\operatorname{Lip}^{2}(H, \mathbb{R})} \max \left\{1, \sup _{t \in[0, T]} \mathbb{E}\left[\left\|X_{t}\right\|_{H}^{3}\right]\right\} \\
& \cdot\left[\frac{1}{T^{\rho}}+\frac{T^{(1-\rho-\vartheta)}\left[\|F\|_{L^{0}{ }^{0}\left(H, H_{-\vartheta}\right)}+\|B\|_{\operatorname{Lip}^{0}\left(H, H S\left(U, H_{-\vartheta / 2}\right)\right)}^{2}\right]}{(1-\rho-\vartheta)}\right]\left\|P_{\mathbb{H} \backslash I}\right\|_{L\left(H, H_{-\rho}\right)} .
\end{aligned}
$$


Proof. Throughout this proof let $\mathbb{U} \subseteq U$ be an orthonormal basis of $U$ and let $B^{b} \in$ $C\left(H, H_{-\vartheta / 2}\right), b \in \mathbb{U}$, be the functions which satisfy for all $b \in \mathbb{U}, v \in H$ that $B^{b}(v)=$ $B(v) b$. Next observe that for all $t \in[0, T]$ it holds $\mathbb{P}$-a.s. that $P_{I}\left(X_{t}\right)=e^{A t} P_{I}(\xi)+$ $\int_{0}^{t} e^{A(t-s)} P_{I} F\left(X_{s}\right) d s+\int_{0}^{t} e^{A(t-s)} P_{I} B\left(X_{s}\right) d W_{s}$. The mild Itô formula in Corollary 2 in Da Prato et al. [14] hence yields that

$$
\begin{aligned}
& \mathbb{E}\left[\varphi\left(X_{T}\right)\right]-\mathbb{E}\left[\varphi\left(P_{I}\left(X_{T}\right)\right)\right]=\mathbb{E}\left[\varphi\left(e^{A T} \xi\right)\right]-\mathbb{E}\left[\varphi\left(e^{A T} P_{I}(\xi)\right)\right] \\
& +\int_{0}^{T} \mathbb{E}\left[\varphi^{\prime}\left(e^{A(T-t)} X_{t}\right) e^{A(T-t)} F\left(X_{t}\right)\right]-\mathbb{E}\left[\varphi^{\prime}\left(e^{A(T-t)} P_{I}\left(X_{t}\right)\right) e^{A(T-t)} P_{I} F\left(X_{t}\right)\right] d t \\
& +\frac{1}{2} \sum_{b \in \mathbb{U}} \int_{0}^{T} \mathbb{E}\left[\varphi^{\prime \prime}\left(e^{A(T-t)} X_{t}\right)\left(e^{A(T-t)} B^{b}\left(X_{t}\right), e^{A(T-t)} B^{b}\left(X_{t}\right)\right)\right] d t \\
& -\frac{1}{2} \sum_{b \in \mathbb{U}} \int_{0}^{T} \mathbb{E}\left[\varphi^{\prime \prime}\left(e^{A(T-t)} P_{I}\left(X_{t}\right)\right)\left(e^{A(T-t)} P_{I} B^{b}\left(X_{t}\right), e^{A(T-t)} P_{I} B^{b}\left(X_{t}\right)\right)\right] d t .
\end{aligned}
$$

Next observe that Lemma 1.2 implies that

$$
\left|\mathbb{E}\left[\varphi\left(e^{A T} \xi\right)\right]-\mathbb{E}\left[\varphi\left(e^{A T} P_{I}(\xi)\right)\right]\right| \leq \frac{|\varphi|_{\operatorname{Lip}^{0}(H, \mathbb{R})} \mathbb{E}\left[\|\xi\|_{H}\right]\left\|P_{\mathbb{H} \backslash I}\right\|_{L\left(H, H_{-\rho}\right)}}{T^{\rho}} .
$$

Inequality (30) provides us a bound for the first difference on the right hand side of (29). In the next step we bound the second difference on the right hand side of (29). For this observe that for all $x \in H, t \in[0, T)$ it holds that

$$
\begin{aligned}
& \left|\left[\varphi^{\prime}\left(e^{A(T-t)} x\right)-\varphi^{\prime}\left(e^{A(T-t)} P_{I}(x)\right)\right] e^{A(T-t)} F(x)\right| \\
& \leq \frac{|\varphi|_{\operatorname{Lip}^{1}(H, \mathbb{R})}\left\|P_{\mathbb{H} \backslash I}\right\|_{L\left(H, H_{-\rho}\right)}\|x\|_{H}\|F(x)\|_{H_{-\vartheta}}}{(T-t)^{(\rho+\vartheta)}}
\end{aligned}
$$

and

$$
\left|\varphi^{\prime}\left(e^{A(T-t)} P_{I}(x)\right)\left(\left[\operatorname{Id}_{H}-P_{I}\right] e^{A(T-t)} F(x)\right)\right| \leq \frac{|\varphi|_{\operatorname{Lip}^{0}(H, \mathbb{R})}\left\|P_{\mathbb{H} \backslash I}\right\|_{L\left(H, H_{-\rho}\right)}\|F(x)\|_{H_{-\vartheta}}}{(T-t)^{(\rho+\vartheta)}} .
$$

Combining (31) and (32) proves that

$$
\begin{aligned}
& \left|\int_{0}^{T} \mathbb{E}\left[\varphi^{\prime}\left(e^{A(T-t)} X_{t}\right) e^{A(T-t)} F\left(X_{t}\right)\right] d t-\int_{0}^{T} \mathbb{E}\left[\varphi^{\prime}\left(e^{A(T-t)} P_{I}\left(X_{t}\right)\right) e^{A(T-t)} P_{I} F\left(X_{t}\right)\right] d t\right| \\
& \leq \frac{T^{(1-\rho-\vartheta)}\left\|P_{\mathbb{H} \backslash I}\right\|_{L\left(H, H_{-}\right)} \sup _{t \in[0, T]} \mathbb{E}\left[\left\|X_{t}\right\|_{H}\left\|F\left(X_{t}\right)\right\|_{H_{-\vartheta}}|\varphi|_{\operatorname{Lip}^{1}(H, \mathbb{R})}+\left\|F\left(X_{t}\right)\right\|_{H_{-\vartheta}}|\varphi|_{\operatorname{Lip}^{0}(H, \mathbb{R})}\right]}{(1-\rho-\vartheta)} \\
& \leq \frac{T^{(1-\rho-\vartheta)}\left\|P_{\mathbb{H} \backslash I}\right\|_{L(H, H-\rho)}\|\varphi\|_{\operatorname{Lip}^{1}(H, \mathbb{R})} \sup _{t \in[0, T]} \max \left\{\mathbb{E}\left[\left\|X_{t}\right\|_{H}\left\|F\left(X_{t}\right)\right\|_{H_{-\vartheta}}\right], \mathbb{E}\left[\left\|F\left(X_{t}\right)\right\|_{H_{-\vartheta}}\right]\right\}}{(1-\rho-\vartheta)} \\
& \leq \frac{T^{(1-\rho-\vartheta)}\left\|P_{\mathbb{H} \backslash I}\right\|_{L\left(H, H_{-\rho}\right)}\|\varphi\|_{\operatorname{Lip}^{1}(H, \mathbb{R})}\|F\|_{\operatorname{Lip}^{0}\left(H, H_{-\vartheta}\right)} \max \left\{1, \sup _{t \in[0, T]} \mathbb{E}\left[\left\|X_{t}\right\|_{H}^{2}\right]\right\}}{(1-\rho-\vartheta)}
\end{aligned}
$$

Inequality (33) provides us a bound for the second difference on the right hand side of (29). Next we bound the third difference on the right hand side of (29). To this end note that for all $x \in H, t \in[0, T)$ it holds that

$$
\begin{aligned}
& \left|\sum_{b \in \mathbb{U}}\left[\varphi^{\prime \prime}\left(e^{A(T-t)} x\right)-\varphi^{\prime \prime}\left(e^{A(T-t)} P_{I}(x)\right)\right]\left(e^{A(T-t)} B^{b}(x), e^{A(T-t)} B^{b}(x)\right)\right| \\
& \leq \frac{|\varphi|_{\operatorname{Lip}^{2}(H, \mathbb{R})}\|B(x)\|_{H S\left(U, H_{-\vartheta / 2}\right)}^{2}\|x\|_{H}\left\|P_{\mathbb{H} \backslash I}\right\|_{L\left(H, H_{-\rho}\right)}}{(T-t)^{(\rho+\vartheta)}}
\end{aligned}
$$


and

$$
\begin{aligned}
& \left|\sum_{b \in \mathbb{U}} \varphi^{\prime \prime}\left(e^{A(T-t)} P_{I}(x)\right)\left(\left[\operatorname{Id}_{H}+P_{I}\right] e^{A(T-t)} B^{b}(x),\left[\operatorname{Id}_{H}-P_{I}\right] e^{A(T-t)} B^{b}(x)\right)\right| \\
& \leq \frac{2|\varphi|_{\operatorname{Lip}^{1}(H, \mathbb{R})}\|B(x)\|_{H S\left(U, H_{-\vartheta / 2}\right.}^{2}\left\|P_{\mathbb{H} \backslash I}\right\|_{L\left(H, H_{-\rho}\right)}}{(T-t)^{(\rho+\vartheta)}} .
\end{aligned}
$$

Combining (34) and (35) proves that

$$
\begin{aligned}
& \mid \frac{1}{2} \sum_{b \in \mathbb{U}} \int_{0}^{T} \mathbb{E}\left[\varphi^{\prime \prime}\left(e^{A(T-t)} X_{t}\right)\left(e^{A(T-t)} B^{b}\left(X_{t}\right), e^{A(T-t)} B^{b}\left(X_{t}\right)\right)\right] d t \\
& -\frac{1}{2} \sum_{b \in \mathbb{U}} \int_{0}^{T} \mathbb{E}\left[\varphi^{\prime \prime}\left(e^{A(T-t)} P_{I}\left(X_{t}\right)\right)\left(e^{A(T-t)} P_{I} B^{b}\left(X_{t}\right), e^{A(T-t)} P_{I} B^{b}\left(X_{t}\right)\right)\right] d t \mid \\
& \leq \frac{\left.T^{(1-\rho-\vartheta)}\left\|P_{\mathbb{H} \backslash I}\right\|_{L(H, H-\rho}\right)\|\varphi\|_{\operatorname{Lip}^{2}(H, \mathbb{R})} \sup _{t \in[0, T]} \max \left\{\mathbb{E}\left[\left\|X_{t}\right\|_{H}\left\|B\left(X_{t}\right)\right\|_{H S(U, H-\vartheta / 2}^{2}\right], \mathbb{E}\left[\left\|B\left(X_{t}\right)\right\|_{H S(U, H-\vartheta / 2}^{2}\right]\right\}}{(1-\rho-\vartheta)} \\
& \leq \frac{T^{(1-\rho-\vartheta)}\left\|P_{\mathbb{H} \backslash I}\right\|_{L\left(H, H_{-\rho}\right)}\|\varphi\|_{\operatorname{Lip}^{2}(H, \mathbb{R})}\|B\|_{\operatorname{Lip}^{0}\left(H, H S\left(U, H_{-\vartheta / 2}\right)\right)}^{2} \max \left\{1, \sup _{t \in[0, T]} \mathbb{E}\left[\left\|X_{t}\right\|_{H}^{3}\right]\right\}}{(1-\rho-\vartheta)} .
\end{aligned}
$$

Combining (29), (30), (33), and (36) finally proves that

$$
\begin{aligned}
& \left|\mathbb{E}\left[\varphi\left(X_{T}\right)\right]-\mathbb{E}\left[\varphi\left(P_{I}\left(X_{T}\right)\right)\right]\right| \leq\|\varphi\|_{\operatorname{Lip}^{2}(H, \mathbb{R})} \max \left\{1, \sup _{t \in[0, T]} \mathbb{E}\left[\left\|X_{t}\right\|_{H}^{3}\right]\right\} \\
& \cdot\left[\frac{1}{T^{\rho}}+\frac{T^{(1-\rho-\vartheta)}\left[\|F\|_{\operatorname{Lip}^{0}\left(H, H_{-\vartheta}\right)}+\|B\|_{\operatorname{Lip}^{0}\left(H, H S\left(U, H_{-\vartheta / 2}\right)\right)}^{2}\right]}{(1-\rho-\vartheta)}\right]\left\|P_{\mathbb{H} \backslash I}\right\|_{L\left(H, H_{-\rho}\right)} .
\end{aligned}
$$

This finishes the proof of Proposition 2.1.

\section{Weak convergence for Galerkin approximations of SEEs with mollified nonlinearities}

In this section we establish weak convergence rates for Galerkin approximations of SEEs with mollified nonlinearities; see Corollary [3.8, Corollary 3.9, and Corollary 3.10 below. Roughly speaking, in the framework of Section 1.4 we establish in Corollary 3.8 below explicit upper bounds for the weak approximation error

$$
\left|\mathbb{E}\left[\varphi\left(X_{T}^{\mathbb{H}}\right)\right]-\mathbb{E}\left[\varphi\left(X_{T}^{I}\right)\right]\right|,
$$

where $I \subseteq \mathbb{H}$ is a set, where $\varphi: H \rightarrow \mathbb{R}$ is a four times continuously Fréchet differentiable function with globally bounded derivatives, and where $X^{\mathbb{H}}:[0, T] \times \Omega \rightarrow H$ and $X^{I}:[0, T] \times \Omega \rightarrow P_{I}(H)$ are appropriate mild solution processes of the SEEs in (146). Here, $X^{I}:[0, T] \times \Omega \rightarrow P_{I}(H)$ is a spectral Galerkin approximation of $X^{\mathbb{H}}:[0, T] \times \Omega \rightarrow H$. We prove Corollary 3.8 by using a decomposition of the weak approximation error as in (10) in Section 1.1 above. Corollary 3.8 is then an immediate consequence of the triangle inequality, of Lemma 3.5 below, and of Lemma 3.7 below. In the proof of Corollary 3.9 we further estimate the right hand side of inequality (220) in Corollary 3.8 to obtain a more explicit upper bound for (38) and the right hand side of (220) in Corollary 3.8, respectively. Corollary 3.9, in particular, enables us to prove inequality (14) in the introduction. In Section 5 below we will use Corollary 3.9 to establish weak convergence rates for Galerkin approximations of SEEs with "non-mollified" nonlinearities. 


\subsection{Regularity properties for solutions of infinite dimensional Kolmogorov equations in Hilbert spaces}

Lemma 3.1. Assume the setting in Section 1.4, let $\varphi \in C_{b}^{4}(H, \mathbb{R}), F \in C_{b}^{4}(H, H)$, $B \in C_{b}^{4}(H, H S(U, H))$, let $X^{x}:[0, T] \times \Omega \rightarrow H, x \in H$, be $\left(\mathcal{F}_{t}\right)_{t \in[0, T]}$-predictable stochastic processes which satisfy for all $x \in H$ that $\sup _{t \in[0, T]} \mathbb{E}\left[\left\|X_{t}^{x}\right\|_{H}^{4}\right]<\infty$ and which satisfy that for all $x \in H, t \in[0, T]$ it holds $\mathbb{P}$-a.s. that

$$
X_{t}^{x}=e^{A t} x+\int_{0}^{t} e^{A(t-s)} F\left(X_{s}^{x}\right) d s+\int_{0}^{t} e^{A(t-s)} B\left(X_{s}^{x}\right) d W_{s},
$$

and let $\phi:[0, T] \times H \rightarrow \mathbb{R}$ be the function which satisfies for all $t \in[0, T], x \in H$ that $\phi(t, x)=\mathbb{E}\left[\varphi\left(X_{t}^{x}\right)\right]$. Then

(i) it holds for all $t \in[0, T]$ that $(H \ni x \mapsto \phi(t, x) \in \mathbb{R}) \in C_{b}^{4}(H, \mathbb{R})$ and

(ii) it holds for all $k \in\{1,2,3,4\}, \delta_{1}, \ldots, \delta_{k} \in(-1 / 2,0]$ with $\sum_{i=1}^{k} \delta_{i}>-1 / 2$ that

$$
\sup _{t \in(0, T]} \sup _{x \in H} \sup _{v_{1}, \ldots, v_{k} \in H \backslash\{0\}}\left[\frac{\left|\left(\frac{\partial^{k}}{\partial x^{k}} \phi\right)(t, x)\left(v_{1}, \ldots, v_{k}\right)\right|}{t^{\left(\delta_{1}+\ldots+\delta_{k}\right)}\left\|v_{1}\right\|_{H_{\delta_{1}}} \cdot \ldots \cdot\left\|v_{k}\right\|_{H_{\delta_{k}}}}\right]<\infty .
$$

Proof. Observe that (39) together with items (iii) \& (vii) of Theorem 3.3 in Andersson et al. [1] (with $T=T, \eta=0, H=H, U=U, V=\mathbb{R}, W=W, A=A, n=4$, $\varphi=\varphi, F=F, B=B, k=k, \delta_{1}=-\delta_{1}, \ldots, \delta_{k}=-\delta_{k}, \alpha=0, \beta=0$ for $\left(\delta_{1}, \ldots, \delta_{k}\right) \in$ $\left\{\left(x_{1}, \ldots, x_{k}\right) \in(-1 / 2,0]^{k}: \sum_{i=1}^{k} x_{i}>-1 / 2\right\}, k \in\{1,2,3,4\}$ in the notation of Theorem 3.3 in [1]) establishes items (ii)-(iii) above. The proof of Lemma 3.1 is thus completed.

In the following we add some comments to Lemma 3.1. Lemma 3.1 is used in the proof of Lemma 3.7 below to establish essentially sharp weak convergence rates. As demonstrated above in the proof of Lemma 3.1. Lemma 3.1 is an immediate consequence of Theorem 3.3 in Andersson et al. [1. Theorem 3.3 in Andersson et al. [1], in particular, establishes a similar result as Lemma 3.1 but under the more general hypothesis that there exists a natural number $n \in \mathbb{N}$ such that $F$ and $B$ are $n$-times continuously Fréchet differentiable with globally bounded derivatives. However, in the proof of Lemma 3.7 below we merely employ estimates of the form (40) for the first four derivatives of the generalized solution $\phi(t, x)=\mathbb{E}\left[\varphi\left(X_{t}^{x}\right)\right],(t, x) \in[0, T] \times H$, of the Kolmogorov equation associated to (39) and, therefore, we restrict ourselves in Lemma 3.1 above to the case $n=4$. Results related to (40) can, e.g., be found in Debussche [19, Lemmas 4.4-4.6] and in Wang \& Gan [50, Lemma 3.3]. In particular, very roughly speaking, Lemmas 4.4-4.5 in [19] establish (40) for all $\delta_{1}, \delta_{k} \in(-1 / 2,0], k \in\{1,2\}$ without the constraint that $\delta_{1}+\delta_{2}>-1 / 2$ but under the additional assumption (2). Moreover, very roughly speaking, Lemma 3.3 in [50] establishes (40) for all $\delta_{1}, \delta_{k} \in(-1,0], k \in\{1,2\}$ with the constraint that $\delta_{1}+\delta_{2}>-1$ in the case of additive noise. Note that condition (2) is obviously satisfied in the case of additive noise. Next we briefly present the idea of the proof of Lemma 3.1 above and of items (iii) \& (vii) of Theorem 3.3 in Andersson et al. 11, respectively. We first combine Vitali's convergence theorem with repeated applications of the chain rule from calculus (cf. Andersson et al. [1, Lemma 2.1, (77), and (100)]) to obtain explicit formulas for the higher order space derivatives of $\phi$ (cf. Andersson et al. [1, Item (v) of Theorem 3.3]) in terms of higher order derivatives of the test function $\varphi$ and in terms of higher order derivative processes associated to (39). Thereafter, we employ Hölder's inequality and suitable estimates for the higher order derivative processes associated to (39) from Andersson et al. [3, Item (ii) of Theorem 2.1] (cf. Andersson et al. [1, (60), (101), and (103)]). The next result, Lemma 3.2 below, is an elementary lemma which provides sufficient conditions for mild solutions of SEEs to be strong solutions. 
Lemma 3.2. Consider the notation in Section 1.3, let $\left(H,\langle\cdot, \cdot\rangle_{H},\|\cdot\|_{H}\right)$ and $\left(U,\langle\cdot, \cdot\rangle_{U},\|\cdot\|_{U}\right)$ be separable $\mathbb{R}$-Hilbert spaces, let $T \in(0, \infty), p \in[2, \infty)$, let $\left(\Omega, \mathcal{F}, \mathbb{P},\left(\mathcal{F}_{t}\right)_{t \in[0, T]}\right)$ be a stochastic basis, let $\left(W_{t}\right)_{t \in[0, T]}$ be an $\operatorname{Id}_{U}$-cylindrical $\left(\Omega, \mathcal{F}, \mathbb{P},\left(\mathcal{F}_{t}\right)_{t \in[0, T]}\right)$-Wiener process, let $A: D(A) \subseteq H \rightarrow H$ be a generator of a strongly continuous analytic semigroup with $\operatorname{spectrum}(A) \subseteq\{z \in \mathbb{C}: \operatorname{Re}(z)<0\}$, let $\left(H_{r},\langle\cdot, \cdot\rangle_{H_{r}},\|\cdot\|_{H_{r}}\right), r \in \mathbb{R}$, be a family of interpolation spaces associated to $-A$, let $\xi \in L^{p}\left(\mathbb{P} ; H_{1}\right)$, let $X:[0, T] \times \Omega \rightarrow H$, $Y:[0, T] \times \Omega \rightarrow H_{1}$, and $Z:[0, T] \times \Omega \rightarrow H S\left(U, H_{1}\right)$ be $\left(\mathcal{F}_{t}\right)_{t \in[0, T]}$-predictable stochastic processes which satisfy that $\int_{0}^{T} \mathbb{E}\left[\left\|Y_{s}\right\|_{H_{1}}^{p}+\left\|Z_{s}\right\|_{H S\left(U, H_{1}\right)}^{p}\right] d s<\infty$ and which satisfy that for all $t \in[0, T]$ it holds $\mathbb{P}$-a.s. that $\int_{0}^{t}\left\|e^{A(t-s)} Y_{s}\right\|_{H}+\left\|e^{A(t-s)} Z_{s}\right\|_{H S(U, H)}^{2} d s<\infty$ and

$$
X_{t}=e^{A t} \xi+\int_{0}^{t} e^{A(t-s)} Y_{s} d s+\int_{0}^{t} e^{A(t-s)} Z_{s} d W_{s}
$$

Then

(i) it holds that

$$
\sup _{t \in[0, T]} \int_{0}^{t} \mathbb{E}\left[\left\|e^{A(t-s)} Y_{s}\right\|_{H_{1}}^{p}+\left\|e^{A(t-s)} Z_{s}\right\|_{H S\left(U, H_{1}\right)}^{p}\right] d s<\infty
$$

(ii) it holds for all $t \in[0, T]$ that

$$
\mathbb{P}\left(X_{t} \in H_{1}\right)=1
$$

(iii) it holds that

$$
\sup _{t \in[0, T]} \mathbb{E}\left[\left\|X_{t} \mathbb{1}_{H_{1}}\left(X_{t}\right)\right\|_{H_{1}}^{p}\right]<\infty
$$

(iv) it holds for all $t \in[0, T]$ that

$$
\limsup _{[0, T] \ni s \rightarrow t}\left\|X_{s} \mathbb{1}_{H_{1}}\left(X_{s}\right)-X_{t} \mathbb{1}_{H_{1}}\left(X_{t}\right)\right\|_{L^{p}\left(\mathbb{P} ; H_{1}\right)}=0,
$$

(v) it holds that

$$
\mathbb{P}\left(\int_{0}^{T}\left\|A X_{s}\right\|_{H_{-1}}+\left\|A\left(X_{s} \mathbb{1}_{H_{1}}\left(X_{s}\right)\right)\right\|_{H}+\left\|Y_{s}\right\|_{H_{1}}+\left\|Z_{s}\right\|_{H S\left(U, H_{1}\right)}^{2} d s<\infty\right)=1,
$$

and

(vi) for all $t \in[0, T]$ it holds $\mathbb{P}$-a.s. that

$$
\begin{aligned}
X_{t} & =\xi+\int_{0}^{t} A\left(X_{s} \mathbb{1}_{H_{1}}\left(X_{s}\right)\right)+Y_{s} d s+\int_{0}^{t} Z_{s} d W_{s} \\
& =\xi+\int_{0}^{t} A X_{s}+Y_{s} d s+\int_{0}^{t} Z_{s} d W_{s} .
\end{aligned}
$$

Proof. Throughout this proof let $h_{N, t} \in[0, T], t \in[0, T], N \in \mathbb{N}$, be the real numbers which satisfy for all $N \in \mathbb{N}, t \in[0, T]$ that $h_{N, t}=\frac{t}{N}$, let $\lfloor\cdot\rfloor_{N, t}: \mathbb{R} \rightarrow \mathbb{R}, t \in[0, T], N \in \mathbb{N}$, be the functions which satisfy for all $N \in \mathbb{N}, t \in[0, T], s \in \mathbb{R}$ that

$$
\lfloor s\rfloor_{N, t}=\max \left((-\infty, s] \cap\left\{0,-h_{N, t}, h_{N, t},-2 h_{N, t}, 2 h_{N, t}, \ldots\right\}\right),
$$

let $\chi \in[0, \infty)$ and $\rho_{r} \in[0, \infty), r \in[0,1]$, be the real numbers which satisfy for all $r \in[0,1]$ that

$$
\chi=\sup _{t \in[0, T]}\left\|e^{A t}\right\|_{L(H)} \quad \text { and } \quad \rho_{r}=\sup _{t \in[0, T]}\left\|(-t A)^{-r}\left(e^{A t}-\operatorname{Id}_{H}\right)\right\|_{L(H)}
$$


(cf., e.g., [42, Lemma 11.36]), and let $\mathcal{X}:[0, T] \times \Omega \rightarrow H_{1}$ be the $\left(\mathcal{F}_{t}\right)_{t \in[0, T] \text {-predictable }}$ stochastic process which satisfies for all $t \in[0, T]$ that

$$
\mathcal{X}_{t}=X_{t} \mathbb{1}_{H_{1}}\left(X_{t}\right)
$$

Observe that for all $t \in[0, T]$ it holds that

$$
\int_{0}^{t} \mathbb{E}\left[\left\|e^{A(t-s)} Y_{s}\right\|_{H_{1}}^{p}+\left\|e^{A(t-s)} Z_{s}\right\|_{H S\left(U, H_{1}\right)}^{p}\right] d s \leq|\chi|^{p} \int_{0}^{t} \mathbb{E}\left[\left\|Y_{s}\right\|_{H_{1}}^{p}+\left\|Z_{s}\right\|_{H S\left(U, H_{1}\right)}^{p}\right] d s .
$$

Therefore, we obtain that

$$
\begin{aligned}
& \sup _{t \in[0, T]} \int_{0}^{t} \mathbb{E}\left[\left\|e^{A(t-s)} Y_{s}\right\|_{H_{1}}^{p}+\left\|e^{A(t-s)} Z_{s}\right\|_{H S\left(U, H_{1}\right)}^{p}\right] d s \\
& \leq|\chi|^{p} \int_{0}^{T} \mathbb{E}\left[\left\|Y_{s}\right\|_{H_{1}}^{p}+\left\|Z_{s}\right\|_{H S\left(U, H_{1}\right)}^{p}\right] d s<\infty .
\end{aligned}
$$

This establishes item (ii). Moreover, Jensen's inequality and the assumption that $p \geq 2$ ensure that

$$
\begin{aligned}
& \int_{0}^{T} \mathbb{E}\left[\left\|Y_{t}\right\|_{H_{1}}\right] d t=T\left[\frac{1}{T} \int_{0}^{T} \mathbb{E}\left[\left(\left\|Y_{t}\right\|_{H_{1}}^{p}\right)^{1 / p}\right] d t\right] \\
& \leq T\left[\frac{1}{T} \int_{0}^{T} \mathbb{E}\left[\left\|Y_{t}\right\|_{H_{1}}^{p}\right] d t\right]^{1 / p}=T^{(1-1 / p)}\left[\int_{0}^{T} \mathbb{E}\left[\left\|Y_{t}\right\|_{H_{1}}^{p}\right] d t\right]^{1 / p}
\end{aligned}
$$

and

$$
\begin{aligned}
& \int_{0}^{T} \mathbb{E}\left[\left\|Z_{t}\right\|_{H S\left(U, H_{1}\right)}^{2}\right] d t=T\left[\frac{1}{T} \int_{0}^{T} \mathbb{E}\left[\left(\left\|Z_{t}\right\|_{H S\left(U, H_{1}\right)}^{p}\right)^{2 / p}\right] d t\right] \\
& \leq T\left[\frac{1}{T} \int_{0}^{T} \mathbb{E}\left[\left\|Z_{t}\right\|_{H S\left(U, H_{1}\right)}^{p}\right] d t\right]^{2 / p}=T^{(1-2 / p)}\left[\int_{0}^{T} \mathbb{E}\left[\left\|Z_{t}\right\|_{H S\left(U, H_{1}\right)}^{p}\right] d t\right]^{2 / p} .
\end{aligned}
$$

Hence, we obtain that

$$
\int_{0}^{T} \mathbb{E}\left[\left\|Y_{t}\right\|_{H_{1}}+\left\|Z_{t}\right\|_{H S\left(U, H_{1}\right)}^{2}\right] d t<\infty
$$

This ensures that for all $t \in[0, T]$ it holds that

$$
\begin{aligned}
& \int_{0}^{t} \mathbb{E}\left[\left\|e^{A(t-s)} Y_{s}\right\|_{H_{1}}+\left\|e^{A(t-s)} Z_{s}\right\|_{H S\left(U, H_{1}\right)}^{2}\right] d s \\
& \leq \int_{0}^{t} \chi \mathbb{E}\left[\left\|Y_{s}\right\|_{H_{1}}\right]+|\chi|^{2} \mathbb{E}\left[\left\|Z_{s}\right\|_{H S\left(U, H_{1}\right)}^{2}\right] d s<\infty .
\end{aligned}
$$

This implies that for all $t \in[0, T]$ it holds that

$$
\mathbb{P}\left(\int_{0}^{t}\left\|e^{A(t-s)} Y_{s}\right\|_{H_{1}}+\left\|e^{A(t-s)} Z_{s}\right\|_{H S\left(U, H_{1}\right)}^{2} d s<\infty\right)=1 .
$$

This, (41), and the assumption that $\xi \in L^{p}\left(\mathbb{P} ; H_{1}\right)$ prove item (iii). Item (iii) and (41) show that for all $t \in[0, T]$ it holds $\mathbb{P}$-a.s. that

$$
\mathcal{X}_{t}=X_{t}=e^{A t} \xi+\int_{0}^{t} e^{A(t-s)} Y_{s} d s+\int_{0}^{t} e^{A(t-s)} Z_{s} d W_{s}
$$


This and the Burkholder-Davis-Gundy type inequality in Lemma 7.7 in Da Prato \& Zabczyk [15] imply that for all $t \in[0, T]$ it holds that

$$
\begin{aligned}
\left\|\mathcal{X}_{t}\right\|_{L^{p}\left(\mathbb{P} ; H_{1}\right)} \leq & \left\|e^{A t} \xi\right\|_{L^{p}\left(\mathbb{P} ; H_{1}\right)}+\int_{0}^{t}\left\|e^{A(t-s)} Y_{s}\right\|_{L^{p}\left(\mathbb{P} ; H_{1}\right)} d s \\
& +\left[\frac{p(p-1)}{2} \int_{0}^{t}\left\|e^{A(t-s)} Z_{s}\right\|_{L^{p}\left(\mathbb{P} ; H S\left(U, H_{1}\right)\right)}^{2} d s\right]^{1 / 2} .
\end{aligned}
$$

Hölder's inequality hence shows that for all $t \in[0, T]$ it holds that

$$
\begin{aligned}
\left\|\mathcal{X}_{t}\right\|_{L^{p}\left(\mathbb{P} ; H_{1}\right)} \leq & \chi\|\xi\|_{L^{p}\left(\mathbb{P} ; H_{1}\right)}+t^{(1-1 / p)}\left[\int_{0}^{t} \mathbb{E}\left[\left\|e^{A(t-s)} Y_{s}\right\|_{H_{1}}^{p}\right] d s\right]^{1 / p} \\
& +\left\{\frac{p(p-1) t^{(1-2 / p)}}{2}\left[\int_{0}^{t} \mathbb{E}\left[\left\|e^{A(t-s)} Z_{s}\right\|_{H S\left(U, H_{1}\right)}^{p}\right] d s\right]^{2 / p}\right\}^{1 / 2}
\end{aligned}
$$

This and item (ii) assure that

$$
\begin{aligned}
& \sup _{t \in[0, T]}\left\|\mathcal{X}_{t}\right\|_{L^{p}\left(\mathbb{P} ; H_{1}\right)} \\
& \leq \chi\|\xi\|_{L^{p}\left(\mathbb{P} ; H_{1}\right)}+T^{(1-1 / p)}\left[\sup _{t \in[0, T]} \int_{0}^{t} \mathbb{E}\left[\left\|e^{A(t-s)} Y_{s}\right\|_{H_{1}}^{p}\right] d s\right]^{1 / p} \\
& \quad+\left\{\frac{p(p-1) T^{(1-2 / p)}}{2}\left[\sup _{t \in[0, T]} \int_{0}^{t} \mathbb{E}\left[\left\|e^{A(t-s)} Z_{s}\right\|_{H S\left(U, H_{1}\right)}^{p}\right] d s\right]^{2 / p}\right\}^{1 / 2}<\infty .
\end{aligned}
$$

This establishes item (iii). Next note that for all $s, t \in[0, T]$ with $s \leq t$ it holds $\mathbb{P}$-a.s. that

$$
\int_{0}^{t} e^{A(t-r)} Y_{r} d r-\int_{0}^{s} e^{A(s-r)} Y_{r} d r=\int_{0}^{t}\left(e^{A(t-r)}-\mathbb{1}_{[0, s]}(r) e^{A \max \{s-r, 0\}}\right) Y_{r} d r .
$$

This and Hölder's inequality show that for all $t, \tau \in[0, T]$ it holds that

$$
\begin{aligned}
& \left\|\int_{0}^{t} e^{A(t-s)} Y_{s} d s-\int_{0}^{\tau} e^{A(\tau-s)} Y_{s} d s\right\|_{L^{p}\left(\mathbb{P} ; H_{1}\right)} \\
& \leq \int_{0}^{\max \{t, \tau\}}\left\|\left(e^{A(\max \{t, \tau\}-s)}-\mathbb{1}_{[0, \min \{t, \tau\}]}(s) e^{A \max \{\min \{t, \tau\}-s, 0\}}\right) Y_{s}\right\|_{L^{p}\left(\mathbb{P} ; H_{1}\right)} d s \\
& \leq T^{(1-1 / p)}\left[\int _ { 0 } ^ { \operatorname { m a x } \{ t , \tau \} } \mathbb { E } \left[\|\left(e^{A(\max \{t, \tau\}-s)}\right.\right.\right. \\
& \left.\left.\left.\quad-\mathbb{1}_{[0, \min \{t, \tau\}]}(s) e^{A \max \{\min \{t, \tau\}-s, 0\}}\right) Y_{s} \|_{H_{1}}^{p}\right] d s\right]^{1 / p} \\
& =T^{(1-1 / p)}\left[\int _ { 0 } ^ { T } \mathbb { E } \left[\mathbb{1}_{[0, \max \{t, \tau\}]}(s) \|\left(e^{A \max \{\max \{t, \tau\}-s, 0\}}\right.\right.\right. \\
& \left.\left.\left.\quad-\mathbb{1}_{[0, \min \{t, \tau\}]}(s) e^{A \max \{\min \{t, \tau\}-s, 0\}}\right) Y_{s} \|_{H_{1}}^{p}\right] d s\right]^{1 / p} .
\end{aligned}
$$

Moreover, observe that for all $s, t, \tau \in[0, T]$ it holds that

$$
\begin{aligned}
& \mathbb{1}_{[0, \max \{t, \tau\}]}(s)\left\|\left(e^{A \max \{\max \{t, \tau\}-s, 0\}}-\mathbb{1}_{[0, \min \{t, \tau\}]}(s) e^{A \max \{\min \{t, \tau\}-s, 0\}}\right) Y_{s}\right\|_{H_{1}} \\
& \leq 2 \chi\left\|Y_{s}\right\|_{H_{1}} .
\end{aligned}
$$


Next note that for all $t \in[0, \infty), v \in H_{1}$ it holds that

$$
\limsup _{[0, \infty) \ni s \rightarrow t}\left\|\left(e^{A t}-e^{A s}\right) v\right\|_{H_{1}}=0 .
$$

Combining (63) - (65) with Lebesgue's theorem of dominated convergence and the assumption that $\int_{0}^{T} \mathbb{E}\left[\left\|Y_{s}\right\|_{H_{1}}^{p}\right] d s<\infty$ yields that for all $t \in[0, T]$ it holds that

$$
\limsup _{[0, T] \ni \tau \rightarrow t}\left\|\int_{0}^{t} e^{A(t-s)} Y_{s} d s-\int_{0}^{\tau} e^{A(\tau-s)} Y_{s} d s\right\|_{L^{p}\left(\mathbb{P} ; H_{1}\right)}=0 .
$$

In the next step note that for all $s, t \in[0, T]$ with $s \leq t$ it holds $\mathbb{P}$-a.s. that

$$
\begin{aligned}
& \int_{0}^{t} e^{A(t-r)} Z_{r} d W_{r}-\int_{0}^{s} e^{A(s-r)} Z_{r} d W_{r} \\
& =\int_{0}^{t}\left(e^{A(t-r)}-\mathbb{1}_{[0, s]}(r) e^{A \max \{s-r, 0\}}\right) Z_{r} d W_{r} .
\end{aligned}
$$

This, Hölder's inequality, and the Burkholder-Davis-Gundy type inequality in Lemma 7.7 in Da Prato \& Zabczyk [15] show that for all $t, \tau \in[0, T]$ it holds that

$$
\begin{aligned}
\| & \int_{0}^{t} e^{A(t-s)} Z_{s} d W_{s}-\int_{0}^{\tau} e^{A(\tau-s)} Z_{s} d W_{s} \|_{L^{p}\left(\mathbb{P} ; H_{1}\right)} \\
\leq & {\left[\frac{p(p-1)}{2}\right]^{1 / 2}\left[\int_{0}^{\max \{t, \tau\}} \|\left(e^{A(\max \{t, \tau\}-s)}\right.\right.} \\
& \left.\left.-\mathbb{1}_{[0, \min \{t, \tau\}]}(s) e^{A \max \{\min \{t, \tau\}-s, 0\}}\right) Z_{s} \|_{L^{p}\left(\mathbb{P} ; H S\left(U, H_{1}\right)\right)}^{2} d s\right]^{1 / 2} \\
\leq & {\left[\frac{p(p-1) T^{(1-2 / p)}}{2}\right]^{1 / 2}\left[\int _ { 0 } ^ { \operatorname { m a x } \{ t , \tau \} } \mathbb { E } \left[\|\left(e^{A(\max \{t, \tau\}-s)}\right.\right.\right.} \\
& \left.\left.\left.-\mathbb{1}_{[0, \min \{t, \tau\}]}(s) e^{A \max \{\min \{t, \tau\}-s, 0\}}\right) Z_{s} \|_{H S\left(U, H_{1}\right)}^{p}\right] d s\right]^{1 / p} \\
= & {\left[\frac{p(p-1) T^{(1-2 / p)}}{2}\right]^{1 / 2}\left[\int _ { 0 } ^ { T } \mathbb { E } \left[\mathbb{1}_{[0, \max \{t, \tau\}]}(s) \|\left(e^{A \max \{\max \{t, \tau\}-s, 0\}}\right.\right.\right.} \\
& \left.\left.\left.-\mathbb{1}_{[0, \min \{t, \tau\}]}(s) e^{A \max \{\min \{t, \tau\}-s, 0\}}\right) Z_{s} \|_{H S\left(U, H_{1}\right)}^{p}\right] d s\right]^{1 / p} .
\end{aligned}
$$

Moreover, observe that for all $s, t, \tau \in[0, T]$ it holds that

$$
\begin{aligned}
& \mathbb{1}_{[0, \max \{t, \tau\}]}(s)\left\|\left(e^{A \max \{\max \{t, \tau\}-s, 0\}}-\mathbb{1}_{[0, \min \{t, \tau\}]}(s) e^{A \max \{\min \{t, \tau\}-s, 0\}}\right) Z_{s}\right\|_{H S\left(U, H_{1}\right)} \\
& \leq 2 \chi\left\|Z_{s}\right\|_{H S\left(U, H_{1}\right)} .
\end{aligned}
$$

In addition, note that (65) and Lebesgue's theorem of dominated convergence ensure that for all $t \in[0, \infty), B \in H S\left(U, H_{1}\right)$ and all orthonormal bases $\mathbb{U} \subseteq U$ of $U$ it holds that

$$
\limsup _{[0, \infty) \ni s \rightarrow t}\left\|\left(e^{A t}-e^{A s}\right) B\right\|_{H S\left(U, H_{1}\right)}^{2}=\limsup _{[0, \infty) \ni s \rightarrow t}\left[\sum_{u \in \mathbb{U}}\left\|\left(e^{A t}-e^{A s}\right) B u\right\|_{H_{1}}^{2}\right]=0 .
$$

Therefore, we obtain for all $t \in[0, T], B \in H S\left(U, H_{1}\right)$ that

$$
\limsup _{[0, T] \ni s \rightarrow t}\left\|\left(e^{A t}-e^{A s}\right) B\right\|_{H S\left(U, H_{1}\right)}^{p}=0 .
$$


Combining (68) with (69), Lebesgue's theorem of dominated convergence, and the assumption that $\int_{0}^{T} \mathbb{E}\left[\left\|Z_{s}\right\|_{H S\left(U, H_{1}\right)}^{p}\right] d s<\infty$ hence yields that for all $t \in[0, T]$ it holds that

$$
\limsup _{[0, T] \ni \tau \rightarrow t}\left\|\int_{0}^{t} e^{A(t-s)} Z_{s} d W_{s}-\int_{0}^{\tau} e^{A(\tau-s)} Z_{s} d W_{s}\right\|_{L^{p}\left(\mathbb{P} ; H_{1}\right)}=0 .
$$

In addition, note that (65) and Lebesgue's theorem of dominated convergence ensure that for all $t \in[0, T]$ it holds that

$$
\limsup _{[0, T] \ni s \rightarrow t}\left\|\left(e^{A t}-e^{A s}\right) \xi\right\|_{L^{p}\left(\mathbb{P} ; H_{1}\right)}=0 .
$$

Combining this, (66), and (172) with (58) establishes item (iv). Next note that items (ii)(iii) imply that

$$
\begin{aligned}
& \int_{0}^{T} \mathbb{E}\left[\left\|A \mathcal{X}_{s}\right\|_{H}\right]+\mathbb{E}\left[\left\|A X_{s}\right\|_{H_{-1}}\right] d s=\int_{0}^{T} \mathbb{E}\left[\left\|\mathcal{X}_{s}\right\|_{H_{1}}\right]+\mathbb{E}\left[\left\|X_{s}\right\|_{H}\right] d s \\
& =\int_{0}^{T} \mathbb{E}\left[\left\|\mathcal{X}_{s}\right\|_{H_{1}}\right]+\mathbb{E}\left[\left\|\mathcal{X}_{s}\right\|_{H}\right] d s \leq\left(1+\left\|A^{-1}\right\|_{L(H)}\right) \int_{0}^{T} \mathbb{E}\left[\left\|\mathcal{X}_{s}\right\|_{H_{1}}\right] d s \\
& \leq T\left(1+\left\|A^{-1}\right\|_{L(H)}\right) \sup _{t \in[0, T]} \mathbb{E}\left[\left\|\mathcal{X}_{t}\right\|_{H_{1}}\right]<\infty .
\end{aligned}
$$

Combining this with (55) yields that

$$
\mathbb{P}\left(\int_{0}^{T}\left\|A X_{s}\right\|_{H_{-1}}+\left\|A \mathcal{X}_{s}\right\|_{H}+\left\|Y_{s}\right\|_{H_{1}}+\left\|Z_{s}\right\|_{H S\left(U, H_{1}\right)}^{2} d s<\infty\right)=1 .
$$

This proves item (表). It thus remains to establish item (vi). For this let $\mathbb{X}:[0, T] \times \Omega \rightarrow H$ be a stochastic process which satisfies that for all $t \in[0, T]$ it holds $\mathbb{P}$-a.s. that

$$
\mathbb{X}_{t}=X_{t}-\xi-\int_{0}^{t} Y_{s} d s-\int_{0}^{t} Z_{s} d W_{s}
$$

Observe that for all $N \in \mathbb{N}, t \in(0, T]$ it holds $\mathbb{P}$-a.s. that

$$
\begin{aligned}
\mathbb{X}_{t} & =\sum_{n=0}^{N-1}\left(\mathbb{X}_{(n+1) h_{N, t}}-\mathbb{X}_{n h_{N, t}}\right)=\sum_{n=0}^{N-1} \int_{n h_{N, t}}^{(n+1) h_{N, t}}\left(\frac{\mathbb{X}_{(n+1) h_{N, t}}-\mathbb{X}_{n h_{N, t}}}{h_{N, t}}\right) d s \\
& =\sum_{n=0}^{N-1} \int_{n h_{N, t}}^{(n+1) h_{N, t}}\left(\frac{\mathbb{X}_{\lfloor s\rfloor_{N, t}+h_{N, t}}-\mathbb{X}_{\lfloor s\rfloor_{N, t}}}{h_{N, t}}\right) d s \\
& =\int_{0}^{t}\left(\frac{\mathbb{X}_{\lfloor s\rfloor_{N, t}+h_{N, t}}-\mathbb{X}_{\lfloor s\rfloor_{N, t}}}{h_{N, t}}\right) d s
\end{aligned}
$$

This implies that for all $t \in(0, T]$ it holds that

$$
\begin{aligned}
& \left\|\mathbb{X}_{t}-\int_{0}^{t} A \mathcal{X}_{s} d s\right\|_{L^{p(\mathbb{P} ; H)}} \\
& \leq \liminf _{\mathbb{N} \ni N \rightarrow \infty}\left(\int_{0}^{t}\left\|\frac{\mathbb{X}_{\lfloor s\rfloor_{N, t}+h_{N, t}}-\mathbb{X}_{\lfloor s\rfloor_{N, t}}}{h_{N, t}}-A \mathcal{X}_{s}\right\|_{L^{p}(\mathbb{P} ; H)} d s\right) \\
& \leq \liminf _{\mathbb{N} \ni N \rightarrow \infty}\left(\int_{0}^{t}\left\|\frac{\mathbb{X}_{\lfloor s\rfloor_{N, t}+h_{N, t}}-\mathbb{X}_{\lfloor s\rfloor_{N, t}}}{h_{N, t}}-A \mathcal{X}_{\lfloor s\rfloor_{N, t}}\right\|_{L^{p}(\mathbb{P} ; H)} d s\right) \\
& \quad+\liminf _{\mathbb{N} \ni N \rightarrow \infty}\left(\int_{0}^{t}\left\|A\left(\mathcal{X}_{\lfloor s\rfloor_{N, t}}-\mathcal{X}_{s}\right)\right\|_{L^{p}(\mathbb{P} ; H)} d s\right) .
\end{aligned}
$$


Next note that items (iii)-(iv) and Lebesgue's theorem of dominated convergence ensure that for all $t \in(0, T]$ it holds that

$$
\limsup _{\mathbb{N} \ni N \rightarrow \infty}\left(\int_{0}^{t}\left\|A\left(\mathcal{X}_{\lfloor s\rfloor_{N, t}}-\mathcal{X}_{s}\right)\right\|_{L^{p}(\mathbb{P} ; H)} d s\right)=0 .
$$

Moreover, observe that item (iii) and (41) imply that for all $s, t \in[0, T]$ with $s \leq t$ it holds $\mathbb{P}$-a.s. that

$$
\begin{aligned}
X_{t} & =e^{A(t-s)} X_{s}+\int_{s}^{t} e^{A(t-r)} Y_{r} d r+\int_{s}^{t} e^{A(t-r)} Z_{r} d W_{r} \\
& =e^{A(t-s)} \mathcal{X}_{s}+\int_{s}^{t} e^{A(t-r)} Y_{r} d r+\int_{s}^{t} e^{A(t-r)} Z_{r} d W_{r}
\end{aligned}
$$

This and item (iii) show that for all $N \in \mathbb{N}, s, t \in(0, T]$ with $s<t$ it holds $\mathbb{P}$-a.s. that

$$
\begin{aligned}
& \frac{\mathbb{X}_{\lfloor s\rfloor_{N, t}+h_{N, t}}-\mathbb{X}_{\lfloor s\rfloor_{N, t}}-h_{N, t} A \mathcal{X}_{\lfloor s\rfloor_{N, t}}}{h_{N, t}} \\
& =\frac{\left(e^{A h_{N, t}}-\operatorname{Id}_{H}-h_{N, t} A\right) \mathcal{X}_{\lfloor s\rfloor_{N, t}}}{h_{N, t}}+\int_{\lfloor s\rfloor_{N, t}}^{\lfloor s\rfloor_{N, t}+h_{N, t}} \frac{\left(e^{A\left(\lfloor s\rfloor_{N, t}+h_{N, t}-r\right)}-\operatorname{Id}_{H}\right)}{h_{N, t}} Y_{r} d r \\
& \quad+\int_{\lfloor s\rfloor_{N, t}}^{\lfloor s\rfloor_{N, t}+h_{N, t}} \frac{\left(e^{A\left(\lfloor s\rfloor_{N, t}+h_{N, t}-r\right)}-\operatorname{Id}_{H}\right)}{h_{N, t}} Z_{r} d W_{r} .
\end{aligned}
$$

This yields that for all $N \in \mathbb{N}, t \in(0, T]$ it holds that

$$
\begin{aligned}
& \int_{0}^{t} \| \frac{\mathbb{X}_{\lfloor s\rfloor_{N, t}+h_{N, t}}-\mathbb{X}_{\lfloor s\rfloor_{N, t}}-A \mathcal{X}_{\lfloor s\rfloor_{N, t}} \|_{L^{p}(\mathbb{P} ; H)} d s}{h_{N, t}} d s \\
& \leq \int_{0}^{t}\left\|\frac{\left(e^{A h_{N, t}}-\operatorname{Id}_{H}-h_{N, t} A\right) \mathcal{X}_{\lfloor s\rfloor_{N, t}}}{h_{N, t}}\right\|_{L^{p}(\mathbb{P} ; H)} d s \\
& \quad+\int_{0}^{t} \int_{\lfloor s\rfloor_{N, t}}^{\lfloor s\rfloor_{N, t}+h_{N, t}} \frac{\left\|\left(e^{A\left(\lfloor s\rfloor_{N, t}+h_{N, t}-r\right)}-\mathrm{Id}_{H}\right) Y_{r}\right\|_{L^{p}(\mathbb{P} ; H)}}{h_{N, t}} d r d s \\
& \quad+\int_{0}^{t}\left\|\int_{\lfloor s\rfloor_{N, t}}^{\lfloor s\rfloor_{N, t}+h_{N, t}} \frac{\left(e^{A\left(\lfloor s\rfloor_{N, t}+h_{N, t}-r\right)}-\mathrm{Id}_{H}\right)}{h_{N, t}} Z_{r} d W_{r}\right\|_{L^{p}(\mathbb{P} ; H)} d s .
\end{aligned}
$$

Next note that for all $N \in \mathbb{N}, s, t \in(0, T]$ with $s \leq t$ it holds that

$$
\begin{aligned}
& \left\|\frac{\left(e^{A h_{N, t}}-\operatorname{Id}_{H}-h_{N, t} A\right) \mathcal{X}_{\lfloor s\rfloor_{N, t}}}{h_{N, t}}\right\|_{L^{p(\mathbb{P} ; H)}} \\
& \leq\left\|\frac{\left(e^{A h_{N, t}}-\operatorname{Id}_{H}-h_{N, t} A\right)\left(\mathcal{X}_{\lfloor s\rfloor_{N, t}}-\mathcal{X}_{s}\right)}{h_{N, t}}\right\|_{L^{p(\mathbb{P} ; H)}} \\
& +\left\|\frac{\left(e^{A h_{N, t}}-\operatorname{Id}_{H}-h_{N, t} A\right) \mathcal{X}_{s}}{h_{N, t}}\right\|_{L^{p}(\mathbb{P} ; H)} \\
& \leq\left\|\frac{\left(e^{A h_{N, t}}-\operatorname{Id}_{H}\right)\left(\mathcal{X}_{\lfloor s\rfloor_{N, t}}-\mathcal{X}_{s}\right)}{h_{N, t}}\right\|_{L^{p(\mathbb{P} ; H)}}+\left\|\mathcal{X}_{\lfloor s\rfloor_{N, t}}-\mathcal{X}_{s}\right\|_{L^{p}\left(\mathbb{P} ; H_{1}\right)} \\
& +\left\|\frac{\left(e^{A h_{N, t}}-\operatorname{Id}_{H}-h_{N, t} A\right) \mathcal{X}_{s}}{h_{N, t}}\right\|_{L^{p(\mathbb{P} ; H)}} \\
& \leq\left(\rho_{1}+1\right)\left\|\mathcal{X}_{\lfloor s\rfloor_{N, t}}-\mathcal{X}_{s}\right\|_{L^{p}\left(\mathbb{P} ; H_{1}\right)}+\left\|\frac{\left(e^{A h_{N, t}}-\operatorname{Id}_{H}-h_{N, t} A\right) \mathcal{X}_{s}}{h_{N, t}}\right\|_{L^{p}(\mathbb{P} ; H)} .
\end{aligned}
$$


In addition, observe that the fact that $\forall v \in H_{1}: \lim \sup _{(0, \infty) \ni h \rightarrow 0}\left\|\frac{\left(e^{A h}-\operatorname{Id}_{H}-h A\right) v}{h}\right\|_{H}=0$ assures that for all $s, t \in(0, T]$ with $s \leq t$ it holds that

$$
\limsup _{\mathbb{N} \ni N \rightarrow \infty}\left\|\frac{\left(e^{A h_{N, t}}-\operatorname{Id}_{H}-h_{N, t} A\right) \mathcal{X}_{s}}{h_{N, t}}\right\|_{H}=0 .
$$

Next observe that for all $N \in \mathbb{N}, s, t \in(0, T]$ with $s \leq t$ it holds that

$$
\left\|\frac{\left(e^{A h_{N, t}}-\operatorname{Id}_{H}-h_{N, t} A\right) \mathcal{X}_{s}}{h_{N, t}}\right\|_{H} \leq\left(\rho_{1}+1\right)\left\|\mathcal{X}_{s}\right\|_{H_{1}} .
$$

This, (84), item (iii), and Lebesgue's theorem of dominated convergence ensure that for all $s, t \in(0, T]$ with $s \leq t$ it holds that

$$
\limsup _{\mathbb{N} \ni N \rightarrow \infty}\left\|\frac{\left(e^{A h_{N, t}}-\operatorname{Id}_{H}-h_{N, t} A\right) \mathcal{X}_{s}}{h_{N, t}}\right\|_{L^{p}(\mathbb{P} ; H)}=0 .
$$

Combining (83) with (86) and item (iv) shows that for all $s, t \in(0, T]$ with $s \leq t$ it holds that

$$
\limsup _{\mathbb{N} \ni N \rightarrow \infty}\left\|\frac{\left(e^{A h_{N, t}}-\operatorname{Id}_{H}-h_{N, t} A\right) \mathcal{X}_{\lfloor s\rfloor_{N, t}}}{h_{N, t}}\right\|_{L^{p}(\mathbb{P} ; H)}=0 .
$$

This, (85), item (iii), and Lebesgue's theorem of dominated convergence yield that for all $t \in(0, T]$ it holds that

$$
\limsup _{\mathbb{N} \ni N \rightarrow \infty}\left(\int_{0}^{t}\left\|\frac{\left(e^{A h_{N, t}}-\operatorname{Id}_{H}-h_{N, t} A\right) \mathcal{X}_{\lfloor s\rfloor_{N, t}}}{h_{N, t}}\right\|_{L^{p}(\mathbb{P} ; H)} d s\right)=0 .
$$

Furthermore, observe that for all $N \in \mathbb{N}, s, t \in(0, T]$ with $s<t$ it holds that

$$
\begin{aligned}
& \int_{\lfloor s\rfloor_{N, t}}^{\lfloor s\rfloor_{N, t}+h_{N, t}} \frac{\left\|\left(e^{A\left(\lfloor s\rfloor_{N, t}+h_{N, t}-r\right)}-\operatorname{Id}_{H}\right) Y_{r}\right\|_{L^{p}(\mathbb{P} ; H)}}{h_{N, t}} d r \\
& \leq \rho_{1} \int_{\lfloor s\rfloor_{N, t}}^{\lfloor s\rfloor_{N, t}+h_{N, t}} \frac{\left(\lfloor s\rfloor_{N, t}+h_{N, t}-r\right)\left\|Y_{r}\right\|_{L^{p}\left(\mathbb{P} ; H_{1}\right)}}{h_{N, t}} d r \\
& \leq \rho_{1} \int_{\lfloor s\rfloor_{N, t}}^{\lfloor s\rfloor_{N, t}+h_{N, t}}\left\|Y_{r}\right\|_{L^{p}\left(\mathbb{P} ; H_{1}\right)} d r=\rho_{1} \int_{0}^{t} \mathbb{1}_{\left(\lfloor s\rfloor_{N, t},\lfloor s\rfloor_{N, t}+h_{N, t}\right)}(r)\left\|Y_{r}\right\|_{L^{p}\left(\mathbb{P} ; H_{1}\right)} d r .
\end{aligned}
$$

In addition, note that Hölder's inequality and the assumption that $\int_{0}^{T} \mathbb{E}\left[\left\|Y_{t}\right\|_{H_{1}}^{p}+\left\|Z_{t}\right\|_{H S\left(U, H_{1}\right)}^{p}\right] d t$ $<\infty$ assure that

$$
\int_{0}^{T}\left\|Y_{t}\right\|_{L^{p}\left(\mathbb{P} ; H_{1}\right)}+\left\|Z_{t}\right\|_{L^{p}\left(\mathbb{P} ; H S\left(U, H_{1}\right)\right)}^{2} d t<\infty .
$$

This, Lebesgue's theorem of dominated convergence, and (89) imply that for all $t \in(0, T]$ it holds that

$$
\begin{aligned}
& \limsup _{\mathbb{N} \ni N \rightarrow \infty}\left(\int_{0}^{t} \int_{\lfloor s\rfloor_{N, t}}^{\lfloor s\rfloor_{N, t}+h_{N, t}} \frac{\left\|\left(e^{A\left(\lfloor s\rfloor_{N, t}+h_{N, t}-r\right)}-\operatorname{Id}_{H}\right) Y_{r}\right\|_{L^{p}(\mathbb{P} ; H)}}{h_{N, t}} d r d s\right) \\
& \leq \rho_{1} \limsup _{\mathbb{N} \ni N \rightarrow \infty} \int_{0}^{t} \int_{0}^{t} \mathbb{1}_{\left(\lfloor s\rfloor_{N, t},\lfloor s\rfloor_{N, t}+h_{N, t}\right)}(r)\left\|Y_{r}\right\|_{L^{p}\left(\mathbb{P} ; H_{1}\right)} d r d s=0 .
\end{aligned}
$$


Next observe that the Burkholder-Davis-Gundy type inequality in Lemma 7.7 in Da Prato \& Zabczyk [15] shows that for all $N \in \mathbb{N}, s, t \in(0, T]$ with $s<t$ it holds that

$$
\begin{aligned}
& \left\|\int_{\lfloor s\rfloor_{N, t}}^{\lfloor s\rfloor_{N, t}+h_{N, t}} \frac{\left(e^{A\left(\lfloor s\rfloor_{N, t}+h_{N, t}-r\right)}-\mathrm{Id}_{H}\right)}{h_{N, t}} Z_{r} d W_{r}\right\|_{L^{p}(\mathbb{P} ; H)} \\
& \leq\left[\frac{p(p-1)}{2} \int_{\lfloor s\rfloor_{N, t}}^{\lfloor s\rfloor_{N, t}+h_{N, t}} \frac{\left\|\left(e^{A\left(\lfloor s\rfloor_{N, t}+h_{N, t}-r\right)}-\mathrm{Id}_{H}\right) Z_{r}\right\|_{L^{p}(\mathbb{P} ; H S(U, H))}^{2}}{\left|h_{N, t}\right|^{2}} d r\right]^{1 / 2} \\
& \leq\left[\frac{p(p-1)\left|\rho_{1}\right|^{2}}{2} \int_{\lfloor s\rfloor_{N, t}}^{\lfloor s\rfloor_{N, t}+h_{N, t}} \frac{\left(\lfloor s\rfloor_{N, t}+h_{N, t}-r\right)^{2}\left\|Z_{r}\right\|_{L^{p}\left(\mathbb{P} ; H S\left(U, H_{1}\right)\right)}^{2}}{\left|h_{N, t}\right|^{2}} d r\right]^{1 / 2} \\
& \leq\left[\frac{p(p-1)\left|\rho_{1}\right|^{2}}{2} \int_{\lfloor s\rfloor_{N, t}}^{\lfloor\rfloor_{N, t}+h_{N, t}}\left\|Z_{r}\right\|_{L^{p}\left(\mathbb{P} ; H S\left(U, H_{1}\right)\right)}^{2} d r\right]^{1 / 2} \\
& =\left[\frac{p(p-1)\left|\rho_{1}\right|^{2}}{2} \int_{0}^{t} \mathbb{1}_{\left(\lfloor s\rfloor_{N, t},\lfloor s\rfloor_{N, t}+h_{N, t}\right)}(r)\left\|Z_{r}\right\|_{L^{p}\left(\mathbb{P} ; H S\left(U, H_{1}\right)\right)}^{2} d r\right]^{1 / 2} .
\end{aligned}
$$

Hölder's inequality hence implies that for all $N \in \mathbb{N}, t \in(0, T]$ it holds that

$$
\begin{aligned}
& \int_{0}^{t}\left\|\int_{\lfloor s\rfloor_{N, t}\lfloor s\rfloor_{N, t}+h_{N, t}} \frac{\left(e^{A\left(\lfloor s\rfloor_{N, t}+h_{N, t}-r\right)}-\operatorname{Id}_{H}\right)}{h_{N, t}} Z_{r} d W_{r}\right\|_{L^{p}(\mathbb{P} ; H)} d s \\
& \leq\left[\frac{p(p-1)\left|\rho_{1}\right|^{2}}{2}\right]^{1 / 2} \int_{0}^{t}\left[\int_{0}^{t} \mathbb{1}_{\left(\lfloor s\rfloor_{N, t}\lfloor s\rfloor_{N, t}+h_{N, t}\right)}(r)\left\|Z_{r}\right\|_{L^{p}\left(\mathbb{P} ; H S\left(U, H_{1}\right)\right)}^{2} d r\right]^{1 / 2} d s \\
& \leq\left[\frac{p(p-1)\left|\rho_{1}\right|^{2} t}{2} \int_{0}^{t} \int_{0}^{t} \mathbb{1}_{\left(\lfloor s\rfloor_{N, t},\lfloor s\rfloor_{N, t}+h_{N, t}\right)}(r)\left\|Z_{r}\right\|_{L^{p}\left(\mathbb{P} ; H S\left(U, H_{1}\right)\right)}^{2} d r d s\right]^{1 / 2} .
\end{aligned}
$$

This, Lebesgue's theorem of dominated convergence, and (90) ensure that for all $t \in(0, T]$ it holds that

$$
\begin{aligned}
& \limsup _{\mathbb{N} \ni N \rightarrow \infty}\left(\int_{0}^{t}\left\|\int_{\lfloor s\rfloor_{N, t}}^{\lfloor s\rfloor_{N, t}+h_{N, t}} \frac{\left(e^{A\left(\lfloor s\rfloor_{N, t}+h_{N, t}-r\right)}-\mathrm{Id}_{H}\right)}{h_{N, t}} Z_{r} d W_{r}\right\|_{L^{p}(\mathbb{P} ; H)} d s\right) \\
& \leq\left[\frac{p(p-1)\left|\rho_{1}\right|^{2} t}{2} \limsup _{\mathbb{N} \ni N \rightarrow \infty}\left(\int_{0}^{t} \int_{0}^{t} \mathbb{1}_{\left(\lfloor s\rfloor_{N, t},\lfloor s\rfloor_{N, t}+h_{N, t}\right)}(r)\left\|Z_{r}\right\|_{L^{p}\left(\mathbb{P} ; H S\left(U, H_{1}\right)\right)}^{2} d r d s\right)\right]^{1 / 2} \\
& =0 .
\end{aligned}
$$

Putting (82), (88), (91), and (94) together yields that for all $t \in(0, T]$ it holds that

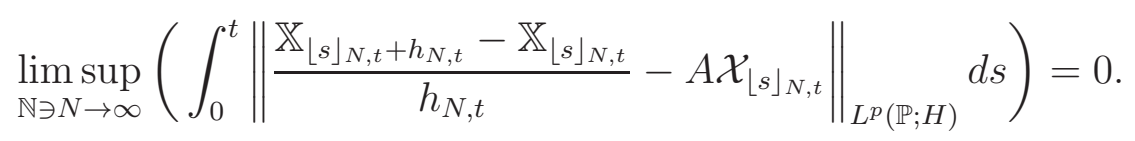

Combining this and (79) with (78) shows that for all $t \in(0, T]$ it holds that

$$
\left\|\mathbb{X}_{t}-\int_{0}^{t} A \mathcal{X}_{s} d s\right\|_{L^{p}(\mathbb{P} ; H)}=0
$$

This, (41), and (76) imply that for all $t \in[0, T]$ it holds $\mathbb{P}$-a.s. that

$$
X_{t}=\xi+\int_{0}^{t} A \mathcal{X}_{s}+Y_{s} d s+\int_{0}^{t} Z_{s} d W_{s}
$$

Moreover, items (iii) \& (四) imply that for all $t \in[0, T]$ it holds $\mathbb{P}$-a.s. that

$$
\xi+\int_{0}^{t} A \mathcal{X}_{s}+Y_{s} d s+\int_{0}^{t} Z_{s} d W_{s}=\xi+\int_{0}^{t} A X_{s}+Y_{s} d s+\int_{0}^{t} Z_{s} d W_{s} .
$$

This, (50), and (97) establish item (vi). The proof of Lemma 3.2 is thus completed. 
The following result, Lemma 3.3 below, can be shown by employing Lemma 3.2 above together with the standard Itô formula in infinite dimensions (cf., e.g., Brzeźniak et al. 99, Theorem 2.4]).

Lemma 3.3. Consider the notation in Section 1.3, let $\left(H,\langle\cdot, \cdot\rangle_{H},\|\cdot\|_{H}\right),\left(U,\langle\cdot, \cdot\rangle_{U},\|\cdot\|_{U}\right)$, and $\left(V,\langle\cdot, \cdot\rangle_{V},\|\cdot\|_{V}\right)$ be separable $\mathbb{R}$-Hilbert spaces, let $T \in(0, \infty)$, let $\left(\Omega, \mathcal{F}, \mathbb{P},\left(\mathcal{F}_{t}\right)_{t \in[0, T]}\right)$ be a stochastic basis, let $\left(W_{t}\right)_{t \in[0, T]}$ be an $\operatorname{Id}_{U}$-cylindrical $\left(\Omega, \mathcal{F}, \mathbb{P},\left(\mathcal{F}_{t}\right)_{t \in[0, T]}\right)$-Wiener process, let $A: D(A) \subseteq H \rightarrow H$ be a generator of a strongly continuous analytic semigroup with $\operatorname{spectrum}(A) \subseteq\{z \in \mathbb{C}: \operatorname{Re}(z)<0\}$, let $\left(H_{r},\langle\cdot, \cdot\rangle_{H_{r}},\|\cdot\|_{H_{r}}\right), r \in \mathbb{R}$, be a family of interpolation spaces associated to $-A$, let $F \in \operatorname{Lip}^{0}\left(H, H_{1}\right), B \in \operatorname{Lip}^{0}\left(H, H S\left(U, H_{1}\right)\right)$, $\varphi \in C_{b}^{2}(H, V)$, let $X^{x}:[0, T] \times \Omega \rightarrow H, x \in H$, be $\left(\mathcal{F}_{t}\right)_{t \in[0, T]}$-predictable stochastic processes which satisfy for all $x \in H$ that $\sup _{t \in[0, T]} \mathbb{E}\left[\left\|X_{t}^{x}\right\|_{H}^{2}\right]<\infty$ and which satisfy that for all $t \in[0, T], x \in H$ it holds $\mathbb{P}$-a.s. that

$$
X_{t}^{x}=e^{A t} x+\int_{0}^{t} e^{A(t-s)} F\left(X_{s}^{x}\right) d s+\int_{0}^{t} e^{A(t-s)} B\left(X_{s}^{x}\right) d W_{s}
$$

and let $u:[0, T] \times H \rightarrow V$ be the function which satisfies for all $t \in[0, T], x \in H$ that $u(t, x)=\mathbb{E}\left[\varphi\left(X_{T-t}^{x}\right)\right]$. Then

(i) it holds for all $x \in H_{1}, t \in[0, T]$ that $\mathbb{P}\left(X_{t}^{x} \in H_{1}\right)=1$,

(ii) it holds for all $p \in[2, \infty)$ that

$$
\sup _{x \in H_{1}} \sup _{t \in[0, T]} \frac{\left(\mathbb{E}\left[\left\|X_{t}^{x} \mathbb{1}_{H_{1}}\left(X_{t}^{x}\right)\right\|_{H_{1}}^{p}\right]\right)^{1 / p}}{\max \left\{1,\|x\|_{H_{1}}\right\}}<\infty,
$$

(iii) it holds for all $x \in H_{1}, t \in[0, T]$ that

$$
\limsup _{[0, T] \times H_{1} \ni(s, y) \rightarrow(t, x)} \mathbb{E}\left[\left\|X_{t}^{x} \mathbb{1}_{H_{1}}\left(X_{t}^{x}\right)-X_{s}^{y} \mathbb{1}_{H_{1}}\left(X_{s}^{y}\right)\right\|_{H_{1}}\right]=0
$$

(iv) it holds for all $x \in H_{1}$ that $([0, T] \ni t \mapsto u(t, x) \in V) \in C^{1}([0, T], V)$, and

(v) it holds that $\left([0, T] \times H_{1} \ni(t, x) \mapsto\left(\frac{\partial}{\partial t} u\right)(t, x) \in V\right) \in C\left([0, T] \times H_{1}, V\right)$.

Proof. Throughout this proof assume w.l.o.g. that $H \neq\{0\}$, let $\mathbb{U} \subseteq U$ be an orthonormal basis of $U$, let $\chi \in[0, \infty)$ be the real number given by

$$
\chi=\sup _{t \in[0, T]}\left\|e^{A t}\right\|_{L(H)},
$$

let $\mathcal{X}^{x}:[0, T] \times \Omega \rightarrow H_{1}, x \in H_{1}$, be the $\left(\mathcal{F}_{t}\right)_{t \in[0, T]}$-predictable stochastic processes which satisfy for all $x \in H_{1}, t \in[0, T]$ that

$$
\mathcal{X}_{t}^{x}=X_{t}^{x} \mathbb{1}_{H_{1}}\left(X_{t}^{x}\right)
$$

and let $\Phi: H_{1} \rightarrow V$ be the function which satisfies for all $x \in H_{1}$ that

$$
\Phi(x)=\varphi^{\prime}(x)(A x+F(x))+\frac{1}{2} \sum_{b \in \mathbb{U}} \varphi^{\prime \prime}(x)(B(x) b, B(x) b) .
$$

Observe that item (i) of Corollary 2.10 in Andersson et al. [2] (with $H=H, U=U$, $T=T, \eta=0, \alpha=0, \beta=0, W=W, A=A, F=(H \ni x \mapsto F(x) \in H), B=(H \ni x \mapsto$ $(U \ni u \mapsto B(x) u \in H) \in H S(U, H))$ in the notation of Corollary 2.10 in [2]) implies that there exist $\left(\mathcal{F}_{t}\right)_{t \in[0, T]}$-predictable stochastic processes $\mathbb{X}^{x}:[0, T] \times \Omega \rightarrow H, x \in H$, which 
satisfy for all $p \in[2, \infty), x \in H$ that $\sup _{t \in[0, T]} \mathbb{E}\left[\left\|\mathbb{X}_{t}^{x}\right\|_{H}^{p}\right]<\infty$ and which satisfy that for all $t \in[0, T], x \in H$ it holds $\mathbb{P}$-a.s. that

$$
\mathbb{X}_{t}^{x}=e^{A t} x+\int_{0}^{t} e^{A(t-s)} F\left(\mathbb{X}_{s}^{x}\right) d s+\int_{0}^{t} e^{A(t-s)} B\left(\mathbb{X}_{s}^{x}\right) d W_{s}
$$

In particular, this implies that for all $x \in H$ it holds that

$$
\sup _{t \in[0, T]} \mathbb{E}\left[\left\|\mathbb{X}_{t}^{x}\right\|_{H}^{2}\right]<\infty
$$

Combining this and (105) with (99) shows that for all $x \in H, t \in[0, T]$ it holds $\mathbb{P}$-a.s. that

$$
X_{t}^{x}=\mathbb{X}_{t}^{x}
$$

(cf., e.g., Da Prato \& Zabczyk [16, Item (i) of Theorem 7.2], Da Prato et al. [14, Proposition 3], or Andersson et al. [2, Item (i) of Theorem 2.9]). Therefore, we obtain that for all $p \in[2, \infty), x \in H, t \in[0, T]$ it holds that

$$
\mathbb{E}\left[\left\|X_{t}^{x}\right\|_{H}^{p}\right]=\mathbb{E}\left[\left\|\mathbb{X}_{t}^{x}\right\|_{H}^{p}\right]
$$

This ensures that for all $p \in[2, \infty), x \in H$ it holds that

$$
\sup _{t \in[0, T]} \mathbb{E}\left[\left\|X_{t}^{x}\right\|_{H}^{p}\right]<\infty .
$$

This, in turn, demonstrates that for all $p \in[2, \infty), x \in H$ it holds that

$$
\begin{aligned}
& \sup _{t \in[0, T]}\left[\left\|F\left(X_{t}^{x}\right)\right\|_{L^{p}\left(\mathbb{P} ; H_{1}\right)}+\left\|B\left(X_{t}^{x}\right)\right\|_{L^{p}\left(\mathbb{P} ; H S\left(U, H_{1}\right)\right)}\right] \\
& \leq\left(\|F\|_{\operatorname{Lip}^{0}\left(H, H_{1}\right)}+\|B\|_{\operatorname{Lip}^{0}\left(H, H_{1}\right)}\right) \sup _{t \in[0, T]} \max \left\{1,\left\|X_{t}^{x}\right\|_{L^{p}(\mathbb{P} ; H)}\right\}<\infty .
\end{aligned}
$$

Hence, we obtain that for all $p \in[2, \infty), x \in H$ it holds that

$$
\int_{0}^{T} \mathbb{E}\left[\left\|F\left(X_{t}^{x}\right)\right\|_{H_{1}}^{p}+\left\|B\left(X_{t}^{x}\right)\right\|_{H S\left(U, H_{1}\right)}^{p}\right] d t<\infty .
$$

This, (99), and items (ii), (iii), (四), and (vi) of Lemma 3.2 imply that

(I) it holds for all $p \in[2, \infty), x \in H_{1}$ that

$$
\sup _{t \in[0, T]} \int_{0}^{t} \mathbb{E}\left[\left\|e^{A(t-s)} F\left(X_{s}^{x}\right)\right\|_{H_{1}}^{p}+\left\|e^{A(t-s)} B\left(X_{s}^{x}\right)\right\|_{H S\left(U, H_{1}\right)}^{p}\right] d s<\infty,
$$

(II) it holds for all $x \in H_{1}, t \in[0, T]$ that

$$
\mathbb{P}\left(X_{t}^{x} \in H_{1}\right)=\mathbb{P}\left(\mathcal{X}_{t}^{x}=X_{t}^{x}\right)=1,
$$

(III) it holds for all $x \in H_{1}$ that

$$
\mathbb{P}\left(\int_{0}^{T}\left\|A X_{s}^{x}\right\|_{H_{-1}}+\left\|F\left(X_{s}^{x}\right)\right\|_{H_{1}}+\left\|B\left(X_{s}^{x}\right)\right\|_{H S\left(U, H_{1}\right)}^{2} d s<\infty\right)=1
$$

and 
(IV) for all $x \in H_{1}, t \in[0, T]$ it holds $\mathbb{P}$-a.s. that

$$
X_{t}^{x}=x+\int_{0}^{t} A X_{s}^{x}+F\left(X_{s}^{x}\right) d s+\int_{0}^{t} B\left(X_{s}^{x}\right) d W_{s}
$$

Observe that item (III) proves item (ii). In the next step we combine (112)-(113) with (99) to obtain that for all $x \in H_{1}, t \in[0, T]$ it holds $\mathbb{P}$-a.s. that

$$
A \mathcal{X}_{t}^{x}=e^{A t} A x+\int_{0}^{t} e^{A(t-s)} A F\left(X_{s}^{x}\right) d s+\int_{0}^{t} e^{A(t-s)} A B\left(X_{s}^{x}\right) d W_{s} .
$$

The Burkholder-Davis-Gundy type inequality in Lemma 7.7 in Da Prato \& Zabczyk [15] therefore shows that for all $p \in[2, \infty), x \in H_{1}, t \in[0, T]$ it holds that

$$
\begin{aligned}
& \left\|A \mathcal{X}_{t}^{x}\right\|_{L^{p}(\mathbb{P} ; H)} \\
& \leq\left\|e^{A t} A x\right\|_{H}+\int_{0}^{t}\left\|e^{A(t-s)} A F\left(X_{s}^{x}\right)\right\|_{L^{p}(\mathbb{P} ; H)} d s \\
& \quad+\left[\frac{p(p-1)}{2} \int_{0}^{t}\left\|e^{A(t-s)} A B\left(X_{s}^{x}\right)\right\|_{L^{p}(\mathbb{P} ; H S(U, H))}^{2} d s\right]^{1 / 2} \\
& \leq \chi\|A x\|_{H}+\int_{0}^{t}\left\|e^{A(t-s)} F\left(X_{s}^{x}\right)\right\|_{L^{p}\left(\mathbb{P} ; H_{1}\right)} d s \\
& \quad+\left[\frac{p(p-1)}{2} \int_{0}^{t}\left\|e^{A(t-s)} B\left(X_{s}^{x}\right)\right\|_{L^{p}\left(\mathbb{P} ; H S\left(U, H_{1}\right)\right)}^{2} d s\right]^{1 / 2} .
\end{aligned}
$$

This, Hölder's inequality, and (112) imply that for all $p \in[2, \infty), x \in H_{1}$ it holds that

$$
\sup _{t \in[0, T]}\left\|A \mathcal{X}_{t}^{x}\right\|_{L^{p}(\mathbb{P} ; H)}<\infty .
$$

Therefore, we obtain for all $p \in[2, \infty), x \in H$ that

$$
\sup _{t \in[0, T]}\left\|A \mathcal{X}_{t}^{A^{-1} x}\right\|_{L^{p}(\mathbb{P} ; H)}<\infty .
$$

Furthermore, observe that (112) and (113) yield that for all $x \in H_{1}, t \in[0, T]$ it holds $\mathbb{P}$-a.s. that

$$
\int_{0}^{t}\left\|e^{A(t-s)} A F\left(\mathcal{X}_{s}^{x}\right)\right\|_{H}+\left\|e^{A(t-s)} A B\left(\mathcal{X}_{s}^{x}\right)\right\|_{H S(U, H)}^{2} d s<\infty
$$

and

$$
\begin{aligned}
\int_{0}^{t} e^{A(t-s)} A F\left(\mathcal{X}_{s}^{x}\right) d s+\int_{0}^{t} e^{A(t-s)} A B\left(\mathcal{X}_{s}^{x}\right) d W_{s} \\
=\int_{0}^{t} e^{A(t-s)} A F\left(X_{s}^{x}\right) d s+\int_{0}^{t} e^{A(t-s)} A B\left(X_{s}^{x}\right) d W_{s} .
\end{aligned}
$$

This and (116) yield that for all $x \in H_{1}, t \in[0, T]$ it holds $\mathbb{P}$-a.s. that

$$
\begin{aligned}
A \mathcal{X}_{t}^{x} & =e^{A t} A x+\int_{0}^{t} e^{A(t-s)} A F\left(\mathcal{X}_{s}^{x}\right) d s+\int_{0}^{t} e^{A(t-s)} A B\left(\mathcal{X}_{s}^{x}\right) d W_{s} \\
& =e^{A t} A x+\int_{0}^{t} e^{A(t-s)} A F\left(A^{-1}\left(A \mathcal{X}_{s}^{x}\right)\right) d s+\int_{0}^{t} e^{A(t-s)} A B\left(A^{-1}\left(A \mathcal{X}_{s}^{x}\right)\right) d W_{s}
\end{aligned}
$$


Hence, we obtain that for all $x \in H, t \in[0, T]$ it holds $\mathbb{P}$-a.s. that

$$
\begin{aligned}
A \mathcal{X}_{t}^{A^{-1} x}= & e^{A t} x+\int_{0}^{t} e^{A(t-s)} A F\left(A^{-1}\left(A \mathcal{X}_{s}^{A^{-1} x}\right)\right) d s \\
& +\int_{0}^{t} e^{A(t-s)} A B\left(A^{-1}\left(A \mathcal{X}_{s}^{A^{-1} x}\right)\right) d W_{s}
\end{aligned}
$$

Moreover, note that for all $x, y \in H$ it holds that

$$
\left\|A F\left(A^{-1} x\right)-A F\left(A^{-1} y\right)\right\|_{H} \leq|F|_{\operatorname{Lip}^{0}\left(H, H_{1}\right)}\left\|A^{-1}\right\|_{L(H)}\|x-y\|_{H}
$$

and

$$
\left\|A B\left(A^{-1} x\right)-A B\left(A^{-1} y\right)\right\|_{H S(U, H)} \leq|B|_{\operatorname{Lip}^{0}\left(H, H S\left(U, H_{1}\right)\right)}\left\|A^{-1}\right\|_{L(H)}\|x-y\|_{H} .
$$

Combining (119) and (123)-(125) with items (i)-(iii) of Corollary 2.10 in Andersson et al. 2] (with $H=H, U=U, T=T, \eta=0, \alpha=0, \beta=0, W=W, A=A, F=(H \ni$ $\left.x \mapsto A F\left(A^{-1} x\right) \in H\right), B=\left(H \ni x \mapsto\left(U \ni u \mapsto A\left[B\left(A^{-1} x\right) u\right] \in H\right) \in H S(U, H)\right)$ in the notation of Corollary 2.10 in [2]) shows that for all $p \in[2, \infty)$ it holds that

$$
\sup _{x \in H} \sup _{t \in(0, T]}\left[\frac{\left\|A \mathcal{X}_{t}^{A^{-1} x}\right\|_{L^{p}(\mathbb{P} ; H)}}{\max \left\{1,\|x\|_{H}\right\}}\right]<\infty
$$

and

$$
\sup _{\substack{x, y \in H, H \\ x \neq y}} \sup _{t \in(0, T]}\left[\frac{\left\|A \mathcal{X}_{t}^{A^{-1} x}-A \mathcal{X}_{t}^{A^{-1} y}\right\|_{L^{p}(\mathbb{P} ; H)}}{\|x-y\|_{H}}\right]<\infty .
$$

Combining (126) and (127) demonstrates that for all $p \in[2, \infty)$ it holds that

$$
\sup _{x \in H_{1}} \sup _{t \in[0, T]}\left[\frac{\left\|\mathcal{X}_{t}^{x}\right\|_{L^{p}\left(\mathbb{P} ; H_{1}\right)}}{\max \left\{1,\|x\|_{H_{1}}\right\}}\right] \leq \sup _{x \in H} \sup _{t \in[0, T]}\left[\frac{\left\|\mathcal{X}_{t}^{A^{-1} x}\right\|_{L^{p}\left(\mathbb{P} ; H_{1}\right)}}{\max \left\{1,\|x\|_{H}\right\}}\right]<\infty
$$

and

$$
\sup _{\substack{x, y \in H_{1}, x \neq y}} \sup _{t \in[0, T]}\left[\frac{\left\|\mathcal{X}_{t}^{x}-\mathcal{X}_{t}^{y}\right\|_{L^{p}\left(\mathbb{P} ; H_{1}\right)}}{\|x-y\|_{H_{1}}}\right] \leq \sup _{\substack{x, y \in H, t \in[0, T] \\ x \neq y}} \sup _{\substack{x \neq y \\ \text { n }}}\left[\frac{\left\|\mathcal{X}_{t}^{A^{-1} x}-\mathcal{X}_{t}^{A^{-1} y}\right\|_{L^{p}\left(\mathbb{P} ; H_{1}\right)}}{\|x-y\|_{H}}\right]<\infty .
$$

Note that item (iii) follows from (128). Moreover, observe that (119), (123)-(125), and, e.g., Proposition 3 in Da Prato et al. [14] (with $T=T, H=H, U=U, Q=\operatorname{Id}_{U}$, $W=W, A=A, \eta=0, \alpha=0, \gamma=0, F=\left(H \ni v \mapsto A F\left(A^{-1} v\right) \in H\right), \beta=0, B=(H \ni$ $\left.v \mapsto\left(U \ni u \mapsto A\left[B\left(A^{-1} v\right) u\right] \in H\right) \in H S(U, H)\right), p=p, \xi=(\Omega \ni \omega \mapsto A x \in H)$ for $x \in H_{1}, p \in[2, \infty)$ in the notation of Proposition 3 in [14]) show that for all $p \in[2, \infty)$, $x \in H_{1}$ it holds that the function

$$
[0, T] \ni t \mapsto A \mathcal{X}_{t}^{x} \in L^{p}(\mathbb{P} ; H)
$$

is continuous. Next note that Hölder's inequality shows that for all $s, t \in[0, T], x, y \in H_{1}$ it holds that

$$
\begin{aligned}
& \mathbb{E}\left[\left\|\mathcal{X}_{s}^{x}-\mathcal{X}_{t}^{y}\right\|_{H_{1}}\right] \\
& \leq \mathbb{E}\left[\left\|\mathcal{X}_{s}^{x}-\mathcal{X}_{s}^{y}\right\|_{H_{1}}\right]+\mathbb{E}\left[\left\|\mathcal{X}_{s}^{y}-\mathcal{X}_{t}^{y}\right\|_{H_{1}}\right] \\
& \leq\left\|\mathcal{X}_{s}^{x}-\mathcal{X}_{s}^{y}\right\|_{L^{2}\left(\mathbb{P} ; H_{1}\right)}+\left\|\mathcal{X}_{s}^{y}-\mathcal{X}_{t}^{y}\right\|_{L^{2}\left(\mathbb{P} ; H_{1}\right)} \\
& \leq\left[\sup _{\substack{u, v \in H_{1}, w \in[0, T] \\
u \neq v}} \frac{\left\|\mathcal{X}_{w}^{u}-\mathcal{X}_{w}^{v}\right\|_{L^{2}\left(\mathbb{P} ; H_{1}\right)}}{\|u-v\|_{H_{1}}}\right]\|x-y\|_{H_{1}}+\left\|\mathcal{X}_{s}^{y}-\mathcal{X}_{t}^{y}\right\|_{L^{2}\left(\mathbb{P} ; H_{1}\right)} .
\end{aligned}
$$


Combining (129)-(131) yields that for all $t \in[0, T], x \in H_{1}$ it holds that

$$
\limsup _{[0, T] \times H_{1} \ni(s, y) \rightarrow(t, x)} \mathbb{E}\left[\left\|\mathcal{X}_{t}^{x}-\mathcal{X}_{s}^{y}\right\|_{H_{1}}\right]=0 .
$$

This establishes item (iii). In the next step we observe that (128) ensures that for all $p \in[2, \infty), x \in H_{1}$ it holds that

$$
\int_{0}^{T} \mathbb{E}\left[\left\|\mathcal{X}_{t}^{x}\right\|_{H_{1}}^{p}\right] d t<\infty
$$

Combining (111), (113), (115), and (133) with the standard Itô formula in infinite dimensions (cf., e.g., Brzeźniak et al. [9, Theorem 2.4]) implies that for all $x \in H_{1}, t \in[0, T]$ it holds $\mathbb{P}$-a.s. that

$$
\varphi\left(\mathcal{X}_{t}^{x}\right)-\varphi(x)=\int_{0}^{t} \Phi\left(\mathcal{X}_{s}^{x}\right) d s+\int_{0}^{t} \varphi^{\prime}\left(\mathcal{X}_{s}^{x}\right) B\left(\mathcal{X}_{s}^{x}\right) d W_{s} .
$$

In addition, observe that for all $x \in H_{1}$ it holds that

$$
\begin{aligned}
\|\Phi(x)\|_{V} \leq & |\varphi|_{C_{b}^{1}(H, V)}\left(\|A x\|_{H}+\|F(x)\|_{H}\right)+\frac{1}{2}|\varphi|_{C_{b}^{2}(H, V)}\|B(x)\|_{H S(U, H)}^{2} \\
\leq & |\varphi|_{C_{b}^{1}(H, V)}\left(\|x\|_{H_{1}}+\|F\|_{\operatorname{Lip}^{0}(H, H)} \max \left\{1,\|x\|_{H}\right\}\right) \\
& +\frac{1}{2}|\varphi|_{C_{b}^{2}(H, V)}\|B\|_{\operatorname{Lip}^{0}(H, H S(U, H))}^{2} \max \left\{1,\|x\|_{H}^{2}\right\} .
\end{aligned}
$$

This, (113), (133), and (134) show that for all $x \in H_{1}, t \in[0, T]$ it holds that $\mathbb{E}\left[\left\|\varphi\left(X_{t}^{x}\right)\right\|_{V}+\right.$ $\left.\left\|\varphi\left(\mathcal{X}_{t}^{x}\right)\right\|_{V}+\int_{0}^{t}\left\|\Phi\left(\mathcal{X}_{s}^{x}\right)\right\|_{V}+\left\|\varphi^{\prime}\left(\mathcal{X}_{s}^{x}\right) B\left(\mathcal{X}_{s}^{x}\right)\right\|_{H S(U, V)}^{2} d s\right]<\infty$ and

$$
\mathbb{E}\left[\varphi\left(X_{t}^{x}\right)\right]=\mathbb{E}\left[\varphi\left(\mathcal{X}_{t}^{x}\right)\right]=\varphi(x)+\int_{0}^{t} \mathbb{E}\left[\Phi\left(\mathcal{X}_{s}^{x}\right)\right] d s .
$$

Moreover, item (iii), the fact that $\Phi \in C\left(H_{1}, V\right)$, and, e.g., Lemma 2.4 in Cox et al. [12] (with $(\Omega, \mathcal{F}, \nu)=(\Omega, \mathcal{F}, \mathbb{P}), E=H_{1}, \mathcal{E}=V, \phi=\Phi, f_{n}=(\Omega \ni \omega \mapsto$ $\left.\mathcal{X}_{t_{n}}^{x_{n}}(\omega) \in H_{1}\right)$ for $n \in \mathbb{N}_{0},\left(\left(t_{m}, x_{m}\right)\right)_{m \in \mathbb{N}_{0}} \in\left\{\left((s, y)=\left(\left(s_{m}, y_{m}\right)\right)_{m \in \mathbb{N}_{0}}: \mathbb{N}_{0} \rightarrow[0, T] \times\right.\right.$ $\left.\left.H_{1}\right): \lim \sup _{\mathbb{N} \ni m \rightarrow \infty}\left|s_{m}-s_{0}\right|+\left\|y_{m}-y_{0}\right\|_{H_{1}}=0\right\}$ in the notation of Lemma 2.4 in [12]) ensure that for all $\left(\left(t_{n}, x_{n}\right)\right)_{n \in \mathbb{N}_{0}} \subseteq[0, T] \times H_{1}$ with $\limsup _{\mathbb{N} \ni n \rightarrow \infty}\left|t_{n}-t_{0}\right|+\left\|x_{n}-x_{0}\right\|_{H_{1}}=0$ it holds that

$$
\limsup _{\mathbb{N} \ni n \rightarrow \infty} \mathbb{E}\left[\min \left\{1,\left\|\Phi\left(\mathcal{X}_{t_{n}}^{x_{n}}\right)-\Phi\left(\mathcal{X}_{t_{0}}^{x_{0}}\right)\right\|_{V}\right\}\right]=0 .
$$

Next note that (135) implies that for all $p \in[2, \infty), x \in H_{1}, t \in[0, T]$ it holds that

$$
\begin{aligned}
& \left\|\Phi\left(\mathcal{X}_{t}^{x}\right)\right\|_{L^{p}(\mathbb{P} ; V)} \\
& \leq|\varphi|_{C_{b}^{1}(H, V)}\left(\left\|\mathcal{X}_{t}^{x}\right\|_{L^{p}\left(\mathbb{P} ; H_{1}\right)}+\|F\|_{\operatorname{Lip}^{0}(H, H)}\left\|\max \left\{1,\left\|\mathcal{X}_{t}^{x}\right\|_{H}\right\}\right\|_{L^{p}(\mathbb{P} ; \mathbb{R})}\right) \\
& \quad+\frac{1}{2}|\varphi|_{C_{b}^{2}(H, V)}\|B\|_{\operatorname{Lip}^{0}(H, H S(U, H))}^{2}\left\|\max \left\{1,\left\|\mathcal{X}_{t}^{x}\right\|_{H}^{2}\right\}\right\|_{L^{p}(\mathbb{P} ; \mathbb{R})} .
\end{aligned}
$$


Item (iii) hence ensures that for all $p \in[2, \infty), x \in H_{1}$ it holds that

$$
\begin{aligned}
& \sup _{\substack{y \in H_{1},\|x-y\|_{H_{1}} \leq 1}} \sup _{t \in[0, T]}\left\|\Phi\left(\mathcal{X}_{t}^{y}\right)\right\|_{L^{p}(\mathbb{P} ; V)} \leq|\varphi|_{C_{b}^{1}(H, V)}\left[\sup _{\substack{y \in H_{1},\|x-y\|_{H_{1}} \leq 1}} \max \left\{1,\|y\|_{H_{1}}\right\}\right] \\
& \cdot\left(\left[\sup _{y \in H_{1}} \sup _{t \in[0, T]} \frac{\left\|\mathcal{X}_{t}^{y}\right\|_{L^{p}\left(\mathbb{P} ; H_{1}\right)}}{\max \left\{1,\|y\|_{H_{1}}\right\}}\right]+\|F\|_{\operatorname{Lip}^{0}(H, H)}\left[\sup _{y \in H_{1}} \sup _{t \in[0, T]} \frac{\left\|\max \left\{1,\left\|\mathcal{X}_{t}^{y}\right\|_{H}\right\}\right\|_{L^{p}(\mathbb{P} ; \mathbb{R})}}{\max \left\{1,\|y\|_{H_{1}}\right\}}\right]\right) \\
& +\frac{1}{2}|\varphi|_{C_{b}^{2}(H, V)}\|B\|_{\operatorname{Lip}^{0}(H, H S(U, H))}^{2}\left[\sup _{\substack{y \in H_{1},\|x-y\|_{H_{1}} \leq 1}} \max \left\{1,\|y\|_{H_{1}}\right\}\right]^{2} \\
& \cdot\left[\sup _{y \in H_{1}} \sup _{t \in[0, T]} \frac{\left\|\max \left\{1,\left\|\mathcal{X}_{t}^{y}\right\|_{H}\right\}\right\|_{L^{2 p}(\mathbb{P} ; \mathbb{R})}}{\max \left\{1,\|y\|_{H_{1}}\right\}}\right]^{2}<\infty \text {. }
\end{aligned}
$$

Combining this, the fact that $\Phi \in C\left(H_{1}, V\right)$, and (137) with, e.g., Proposition 4.5 in Hutzenthaler et al. [28] (with $I=\{\emptyset\}, p=p, V=V, X^{n}=(\{\emptyset\} \times \Omega \ni(\emptyset, \omega) \mapsto$ $\left.\Phi\left(\mathcal{X}_{s_{n}}^{x_{n}}(\omega)\right) \in V\right), q=1$ for $n \in \mathbb{N}_{0},\left(\left(s_{m}, x_{m}\right)\right)_{m \in \mathbb{N}_{0}} \in\left\{\left((t, y)=\left(\left(t_{m}, y_{m}\right)\right)_{m \in \mathbb{N}_{0}}: \mathbb{N}_{0} \rightarrow\right.\right.$ $\left.\left.[0, T] \times H_{1}\right): \lim \sup _{\mathbb{N} \ni m \rightarrow \infty}\left|t_{m}-t_{0}\right|+\left\|y_{m}-y_{0}\right\|_{H_{1}}=0\right\}, p \in[2, \infty)$ in the notation of Proposition 4.5 in [28]) yields that for all $p \in[2, \infty),\left(\left(s_{n}, x_{n}\right)\right)_{n \in \mathbb{N}_{0}} \subseteq[0, T] \times H_{1}$ with $\lim \sup _{\mathbb{N} \ni n \rightarrow \infty}\left|s_{n}-s_{0}\right|+\left\|x_{n}-x_{0}\right\|_{H_{1}}=0$ it holds that

$$
\limsup _{\mathbb{N} \ni n \rightarrow \infty} \mathbb{E}\left[\left\|\Phi\left(\mathcal{X}_{s_{n}}^{x_{n}}\right)-\Phi\left(\mathcal{X}_{s_{0}}^{x_{0}}\right)\right\|_{V}\right]=0
$$

This assures that the function

$$
[0, T] \times H_{1} \ni(s, x) \mapsto \mathbb{E}\left[\Phi\left(\mathcal{X}_{s}^{x}\right)\right] \in V
$$

is continuous. In particular, it holds for all $x \in H_{1}$ that the function

$$
[0, T] \ni s \mapsto \mathbb{E}\left[\Phi\left(\mathcal{X}_{s}^{x}\right)\right] \in V
$$

is continuous. This, (136), the fundamental theorem of calculus, and the fact that

$$
\forall t \in[0, T], x \in H: \quad \mathbb{E}\left[\varphi\left(X_{t}^{x}\right)\right]=u(T-t, x)
$$

show that for all $x \in H_{1}, t \in[0, T]$ it holds that $([0, T] \ni s \mapsto u(s, x) \in V) \in C^{1}([0, T], V)$ and

$$
\left(\frac{\partial}{\partial t} u\right)(t, x)=-\mathbb{E}\left[\Phi\left(\mathcal{X}_{t}^{x}\right)\right] .
$$

This and (141) establish items (iv)-(䜣). The proof of Lemma 3.3 is thus completed.

\subsection{Setting}

Assume the setting in Section 1.4, let $\mathbb{U} \subseteq U$ be an orthonormal basis of $U$, let $\vartheta \in[0,1 / 2)$, $F \in C_{b}^{4}\left(H, H_{2}\right), B \in C_{b}^{4}\left(H, H S\left(U, H_{2}\right)\right), \varphi \in C_{b}^{4}(H, \mathbb{R}), \xi \in L^{4}\left(\left.\mathbb{P}\right|_{\mathcal{F}_{0}} ; H_{2}\right)$, let $\varsigma_{F, B} \in \mathbb{R}$ be given by $\varsigma_{F, B}=\max \left\{1,\|F\|_{C_{b}^{3}\left(H, H_{-\vartheta}\right)}^{2},\|B\|_{C_{b}^{3}\left(H, H S\left(U, H_{-\vartheta / 2}\right)\right.}^{4}\right\}$, let $\left(F_{I}\right)_{I \in \mathcal{P}(\mathbb{H})} \subseteq C(H, H)$, $\left(B_{I}\right)_{I \in \mathcal{P}(\mathbb{H})} \subseteq C(H, H S(U, H)),\left(B^{b}\right)_{b \in \mathbb{U}} \subseteq C(H, H)$, and $\left(B_{I}^{b}\right)_{I \in \mathcal{P}(\mathbb{H}), b \in \mathbb{U}} \subseteq C(H, H)$ satisfy for all $I \in \mathcal{P}(\mathbb{H}), b \in \mathbb{U}, u \in U, v \in H$ that

$$
F_{I}(v)=F\left(P_{I}(v)\right), \quad B_{I}(v) u=B\left(P_{I}(v)\right) u, \quad B^{b}(v)=B(v) b, \quad B_{I}^{b}(v)=B\left(P_{I}(v)\right) b,
$$


let $\left(g_{r}\right)_{r \in[0, \infty)} \subseteq C(H, \mathbb{R})$ satisfy for all $r \in[0, \infty), x \in H$ that $g_{r}(x)=\max \left\{1,\|x\|_{H}^{r}\right\}$, let $X^{I}:[0, T] \times \Omega \rightarrow P_{I}(H), I \in \mathcal{P}(\mathbb{H}), Y^{I}:[0, T] \times \Omega \rightarrow H_{2}, I \in \mathcal{P}(\mathbb{H})$, and $X^{\mathbb{H}, x}:[0, T] \times$ $\Omega \rightarrow H, x \in H$, be $\left(\mathcal{F}_{t}\right)_{t \in[0, T]}$-predictable stochastic processes which satisfy for all $I \in$ $\mathcal{P}(\mathbb{H}), x \in H$ that $\sup _{t \in[0, T]} \mathbb{E}\left[\left\|X_{t}^{I}\right\|_{H}^{4}+\left\|Y_{t}^{I}\right\|_{H_{2}}^{4}+\left\|X_{t}^{\mathbb{H}, x}\right\|_{H}^{4}\right]<\infty$ and which satisfy that for all $t \in[0, T], I \in \mathcal{P}(\mathbb{H}), x \in H$ it holds $\mathbb{P}$-a.s. that

$$
\begin{gathered}
X_{t}^{I}=e^{A t} P_{I}(\xi)+\int_{0}^{t} e^{A(t-s)} P_{I} F\left(X_{s}^{I}\right) d s+\int_{0}^{t} e^{A(t-s)} P_{I} B\left(X_{s}^{I}\right) d W_{s}, \\
Y_{t}^{I}=e^{A t} \xi+\int_{0}^{t} e^{A(t-s)} F_{I}\left(Y_{s}^{I}\right) d s+\int_{0}^{t} e^{A(t-s)} B_{I}\left(Y_{s}^{I}\right) d W_{s}, \\
X_{t}^{\mathbb{H}, x}=e^{A t} x+\int_{0}^{t} e^{A(t-s)} F\left(X_{s}^{\mathbb{H}, x}\right) d s+\int_{0}^{t} e^{A(t-s)} B\left(X_{s}^{\mathbb{H}, x}\right) d W_{s},
\end{gathered}
$$

let $u:[0, T] \times H \rightarrow \mathbb{R}$ be the function which satisfies for all $t \in[0, T], x \in H$ that $u(t, x)=\mathbb{E}\left[\varphi\left(X_{T-t}^{\mathbb{H}, x}\right)\right]$, let $c_{\delta_{1}, \ldots, \delta_{k}} \in[0, \infty], \delta_{1}, \ldots, \delta_{k} \in(-\infty, 0], k \in\{1,2,3,4\}$, be the extended real numbers which satisfy for all $k \in\{1,2,3,4\}, \delta_{1}, \ldots, \delta_{k} \in(-\infty, 0]$ that

$$
c_{\delta_{1}, \ldots, \delta_{k}}=\sup _{t \in[0, T)} \sup _{x \in H} \sup _{v_{1}, \ldots, v_{k} \in H \backslash\{0\}}\left[\frac{\left|\left(\frac{\partial^{k}}{\partial x^{k}} u\right)(t, x)\left(v_{1}, \ldots, v_{k}\right)\right|}{(T-t)^{\left(\delta_{1}+\ldots+\delta_{k}\right)}\left\|v_{1}\right\|_{H_{\delta_{1}}} \cdot \ldots \cdot\left\|v_{k}\right\|_{H_{\delta_{k}}}}\right]
$$

(cf., e.g., item (1i) of Lemma 3.1), and let $\left(K_{r}^{I}\right)_{r \in(0,4], I \in \mathcal{P}(\mathbb{H})} \subseteq[0, \infty)$ satisfy for all $I \in$ $\mathcal{P}(\mathbb{H}), r \in(0,4]$ that $K_{r}^{I}=\sup _{t \in[0, T]} \mathbb{E}\left[g_{r}\left(Y_{t}^{I}\right)\right]$.

\subsection{Weak convergence results}

Lemma 3.4. Assume the setting in Section 3.2. Then it holds for all $k \in\{1,2,3,4\}$, $\delta_{1}, \ldots, \delta_{k} \in(-1 / 2,0]$ with $\sum_{i=1}^{k} \delta_{i}>-1 / 2$ that $c_{\delta_{1}, \ldots, \delta_{k}}<\infty$.

Proof. Throughout this proof let $\phi:[0, T] \times H \rightarrow \mathbb{R}$ be the function which satisfies for all $t \in[0, T], x \in H$ that $\phi(t, x)=\mathbb{E}\left[\varphi\left(X_{t}^{\mathbb{H}, x}\right)\right]$. Note that for all $t \in[0, T], x \in H$ it holds that $u(t, x)=\phi(T-t, x)$. This and item (iii) of Lemma 3.1 (with $\varphi=\varphi, F=(H \ni v \mapsto$ $F(v) \in H), B=(H \ni v \mapsto(U \ni u \mapsto B(v) u \in H) \in H S(U, H)), X^{x}=X^{\mathbb{H}, x}, \phi=\phi$, $k=k, \delta_{1}=\delta_{1}, \ldots, \delta_{k}=\delta_{k}$ for $\left(\delta_{1}, \ldots, \delta_{k}\right) \in\left\{\left(x_{1}, \ldots, x_{k}\right) \in(-1 / 2,0]^{k}: \sum_{i=1}^{k} x_{i}>-1 / 2\right\}$, $k \in\{1,2,3,4\}, x \in H$ in the notation of Lemma 3.1) imply that for all $k \in\{1,2,3,4\}$, $\delta_{1}, \ldots, \delta_{k} \in(-1 / 2,0]$ with $\sum_{i=1}^{k} \delta_{i}>-1 / 2$ it holds that

$$
\begin{aligned}
c_{\delta_{1}, \ldots, \delta_{k}} & =\sup _{t \in[0, T)} \sup _{x \in H} \sup _{v_{1}, \ldots, v_{k} \in H \backslash\{0\}}\left[\frac{\left|\left(\frac{\partial^{k}}{\partial x^{k}} \phi\right)(T-t, x)\left(v_{1}, \ldots, v_{k}\right)\right|}{(T-t)^{\left(\delta_{1}+\ldots+\delta_{k}\right)}\left\|v_{1}\right\|_{H_{\delta_{1}}} \cdot \ldots \cdot\left\|v_{k}\right\|_{H_{\delta_{k}}}}\right] \\
& =\sup _{t \in(0, T]} \sup _{x \in H} \sup _{v_{1}, \ldots, v_{k} \in H \backslash\{0\}}\left[\frac{\left|\left(\frac{\partial^{k}}{\partial x^{k}} \phi\right)(t, x)\left(v_{1}, \ldots, v_{k}\right)\right|}{t^{\left(\delta_{1}+\ldots+\delta_{k}\right)}\left\|v_{1}\right\|_{H_{\delta_{1}}} \cdot \ldots \cdot\left\|v_{k}\right\|_{H_{\delta_{k}}}}\right]<\infty .
\end{aligned}
$$

The proof of Lemma 3.4 is thus completed.

Lemma 3.5. Assume the setting in Section 3.2 and let $\rho \in[0,1-\vartheta), I \in \mathcal{P}(\mathbb{H})$. Then

$$
\left|\mathbb{E}\left[\varphi\left(Y_{T}^{I}\right)\right]-\mathbb{E}\left[\varphi\left(X_{T}^{I}\right)\right]\right| \leq\left[\frac{1}{T^{\rho}}+\frac{2 T^{(1-\rho-\vartheta)}}{(1-\rho-\vartheta)}\right]\|\varphi\|_{C_{b}^{3}(H, \mathbb{R})} \varsigma_{F, B} K_{3}^{I}\left\|P_{\mathbb{H} \backslash I}\right\|_{L\left(H, H_{-\rho}\right)} .
$$


Proof. First of all, note that for all $t \in[0, T]$ it holds $\mathbb{P}$-a.s. that

$$
P_{I}\left(Y_{t}^{I}\right)=e^{A t} P_{I}(\xi)+\int_{0}^{t} e^{A(t-s)} P_{I} F\left(P_{I}\left(Y_{s}^{I}\right)\right) d s+\int_{0}^{t} e^{A(t-s)} P_{I} B\left(P_{I}\left(Y_{s}^{I}\right)\right) d W_{s}
$$

The fact that mild solutions of (146) are within a suitable class of solutions unique up to modifications (see, e.g., item (i) of Theorem 7.2 in Da Prato \& Zabczyk [16] for details) hence ensures that for all $t \in[0, T]$ it holds $\mathbb{P}$-a.s. that $P_{I}\left(Y_{t}^{I}\right)=X_{t}^{I}$. An application of Proposition 2.1 hence proves that

$$
\begin{aligned}
& \left|\mathbb{E}\left[\varphi\left(Y_{T}^{I}\right)\right]-\mathbb{E}\left[\varphi\left(X_{T}^{I}\right)\right]\right| \leq\|\varphi\|_{C_{b}^{3}(H, \mathbb{R})} \max \left\{1, \sup _{t \in[0, T]} \mathbb{E}\left[\left\|Y_{t}^{I}\right\|_{H}^{3}\right]\right\} \\
& \cdot\left[\frac{1}{T^{\rho}}+\frac{T^{(1-\rho-\vartheta)}\left[\|F\|_{C_{b}^{1}\left(H, H_{-\vartheta}\right)}+\|B\|_{C_{b}^{1}\left(H, H S\left(U, H_{-\vartheta / 2}\right)\right.}^{2}\right]}{(1-\rho-\vartheta)}\right]\left\|P_{\mathbb{H} \backslash I}\right\|_{L\left(H, H_{-\rho}\right)} .
\end{aligned}
$$

This completes the proof of Lemma 3.5.

Corollary 3.6. Assume the setting in Section 3.2 and let $I \in \mathcal{P}(\mathbb{H})$. Then

(i) it holds that

$$
\int_{0}^{T} \mathbb{E}\left[\left\|A Y_{t}^{I}\right\|_{H_{1}}+\left\|F_{I}\left(Y_{t}^{I}\right)\right\|_{H_{1}}+\left\|B_{I}\left(Y_{t}^{I}\right)\right\|_{H S\left(U, H_{1}\right)}^{2}\right] d t<\infty
$$

and

(ii) for all $t \in[0, T]$ it holds $\mathbb{P}$-a.s. that

$$
Y_{t}^{I}=\xi+\int_{0}^{t} A Y_{s}^{I}+F_{I}\left(Y_{s}^{I}\right) d s+\int_{0}^{t} B_{I}\left(Y_{s}^{I}\right) d W_{s}
$$

Proof. Observe that for all $p \in[1,4]$ it holds that

$$
\begin{aligned}
& \sup _{t \in[0, T]}\left[\left\|F_{I}\left(Y_{t}^{I}\right)\right\|_{L^{p}\left(\mathbb{P} ; H_{2}\right)}+\left\|B_{I}\left(Y_{t}^{I}\right)\right\|_{L^{p}\left(\mathbb{P} ; H S\left(U, H_{2}\right)\right)}\right] \\
& \leq \sup _{t \in[0, T]}\left[\|F(0)\|_{H_{2}}+\left\|F\left(P_{I}\left(Y_{t}^{I}\right)\right)-F(0)\right\|_{L^{p}\left(\mathbb{P} ; H_{2}\right)}\right] \\
& \quad+\sup _{t \in[0, T]}\left[\|B(0)\|_{H S\left(U, H_{2}\right)}+\left\|B\left(P_{I}\left(Y_{t}^{I}\right)\right)-B(0)\right\|_{L^{p}\left(\mathbb{P} ; H S\left(U, H_{2}\right)\right)}\right] \\
& \leq \sup _{t \in[0, T]}\left[\|F(0)\|_{H_{2}}+|F|_{\operatorname{Lip}^{0}\left(H, H_{2}\right)}\left\|P_{I}\left(Y_{t}^{I}\right)\right\|_{L^{p}(\mathbb{P} ; H)}\right] \\
& \quad+\sup _{t \in[0, T]}\left[\|B(0)\|_{H S\left(U, H_{2}\right)}+|B|_{\operatorname{Lip}^{0}\left(H, H S\left(U, H_{2}\right)\right)}\left\|P_{I}\left(Y_{t}^{I}\right)\right\|_{L^{p}(\mathbb{P} ; H)}\right] \\
& \leq \sup _{t \in[0, T]}\left[\|F(0)\|_{H_{2}}+|F|_{\operatorname{Lip}^{0}\left(H, H_{2}\right)} \max \left\{1,\left\|P_{I}\left(Y_{t}^{I}\right)\right\|_{L^{p}(\mathbb{P} ; H)}\right\}\right] \\
& \quad+\sup _{t \in[0, T]}\left[\|B(0)\|_{H S\left(U, H_{2}\right)}+|B|_{\operatorname{Lip}^{0}\left(H, H S\left(U, H_{2}\right)\right)} \max \left\{1,\left\|P_{I}\left(Y_{t}^{I}\right)\right\|_{L^{p}(\mathbb{P} ; H)}\right\}\right] .
\end{aligned}
$$


Hence, we obtain that for all $p \in[1,4]$ it holds that

$$
\begin{aligned}
& \sup _{t \in[0, T]}\left[\left\|F_{I}\left(Y_{t}^{I}\right)\right\|_{L^{p}\left(\mathbb{P} ; H_{2}\right)}+\left\|B_{I}\left(Y_{t}^{I}\right)\right\|_{L^{p}\left(\mathbb{P} ; H S\left(U, H_{2}\right)\right)}\right] \\
& \leq \sup _{t \in[0, T]}\left[\|F(0)\|_{H_{2}}+|F|_{\operatorname{Lip}^{0}\left(H, H_{2}\right)} \max \left\{1,\left\|Y_{t}^{I}\right\|_{L^{p}(\mathbb{P} ; H)}\right\}\right] \\
& \quad+\sup _{t \in[0, T]}\left[\|B(0)\|_{H S\left(U, H_{2}\right)}+|B|_{\operatorname{Lip}^{0}\left(H, H S\left(U, H_{2}\right)\right)} \max \left\{1,\left\|Y_{t}^{I}\right\|_{L^{p}(\mathbb{P} ; H)}\right\}\right] \\
& \leq \sup _{t \in[0, T]}\left(\left[\|F(0)\|_{H_{2}}+|F|_{\operatorname{Lip}^{0}\left(H, H_{2}\right)}\right] \max \left\{1,\left\|Y_{t}^{I}\right\|_{L^{p}(\mathbb{P} ; H)}\right\}\right) \\
& \quad+\sup _{t \in[0, T]}\left(\left[\|B(0)\|_{H S\left(U, H_{2}\right)}+|B|_{\operatorname{Lip}^{0}\left(H, H S\left(U, H_{2}\right)\right)}\right] \max \left\{1,\left\|Y_{t}^{I}\right\|_{L^{p}(\mathbb{P} ; H)}\right\}\right) \\
& =\left(\|F\|_{\operatorname{Lip}^{0}\left(H, H_{2}\right)}+\|B\|_{\operatorname{Lip}^{0}\left(H, H S\left(U, H_{2}\right)\right)}\right) \sup _{t \in[0, T]} \max \left\{1,\left\|Y_{t}^{I}\right\|_{L^{p}(\mathbb{P} ; H)}\right\}<\infty .
\end{aligned}
$$

This ensures that for all $p \in[1,4]$ it holds that

$$
\int_{0}^{T} \mathbb{E}\left[\left\|F_{I}\left(Y_{t}^{I}\right)\right\|_{H_{2}}^{p}+\left\|B_{I}\left(Y_{t}^{I}\right)\right\|_{H S\left(U, H_{2}\right)}^{p}\right] d t<\infty .
$$

In addition, note that Hölder's inequality and the assumption that $\sup _{t \in[0, T]} \mathbb{E}\left[\left\|Y_{t}^{I}\right\|_{H_{2}}^{4}\right]<$ $\infty$ imply that

$$
\sup _{t \in[0, T]} \mathbb{E}\left[\left\|Y_{t}^{I}\right\|_{H_{2}}\right]<\infty
$$

Therefore, we obtain that

$$
\int_{0}^{T} \mathbb{E}\left[\left\|Y_{t}^{I}\right\|_{H_{2}}\right] d t<\infty .
$$

This and (158) prove item (ii). In the next step we combine (158) with (147) and items (国)(vil) of Lemma 3.2 to obtain that for all $t \in[0, T]$ it holds $\mathbb{P}$-a.s. that

$$
Y_{t}^{I}=\xi+\int_{0}^{t} A Y_{s}^{I}+F_{I}\left(Y_{s}^{I}\right) d s+\int_{0}^{t} B_{I}\left(Y_{s}^{I}\right) d W_{s} .
$$

This establishes item (iii). The proof of Corollary 3.6 is thus completed.

Lemma 3.7. Assume the setting in Section 3.2 and let $\rho \in[0,1-\vartheta), I \in \mathcal{P}(\mathbb{H})$. Then

$$
\begin{aligned}
\mid \mathbb{E}\left[\varphi\left(X_{T}^{\mathbb{H}}\right)\right]- & \mathbb{E}\left[\varphi\left(Y_{T}^{I}\right)\right] \mid \leq \frac{T^{(1-\vartheta-\rho)} \varsigma_{F, B} K_{4}^{I}}{(1-\vartheta-\rho)}\left[1+\frac{9 T^{(1-\vartheta)}}{2(1-\vartheta)}\right]\left\|P_{\mathbb{H} \backslash I}\right\|_{L\left(H, H_{-\rho}\right)} \\
\cdot & {\left[c_{-\vartheta}+c_{-\vartheta, 0}+c_{-\vartheta, 0,0}+c_{-\vartheta / 2,-\vartheta / 2}+c_{-\vartheta / 2,-\vartheta / 2,0}+c_{-\vartheta / 2,-\vartheta / 2,0,0}\right]<\infty }
\end{aligned}
$$

Proof. Throughout this proof let $u_{1,0}:[0, T] \times H_{1} \rightarrow \mathbb{R}$ and $u_{0, k}:[0, T] \times H \rightarrow L^{(k)}(H, \mathbb{R})$, $k \in\{1,2,3,4\}$, be the functions which satisfy for all $k \in\{1,2,3,4\}, t \in[0, T], w \in H_{1}$, $x, v_{1}, \ldots, v_{k} \in H$ that

$$
u_{1,0}(t, w)=\left(\frac{\partial}{\partial t} u\right)(t, w) \quad \text { and } \quad u_{0, k}(t, x)\left(v_{1}, \ldots, v_{k}\right)=\left(\left(\frac{\partial^{k}}{\partial x^{k}} u\right)(t, x)\right)\left(v_{1}, \ldots, v_{k}\right)
$$

(cf., e.g., item (iv) of Lemma 3.3 and item (ii) of Lemma 3.1). Note that items (ii) \& (iii) of Lemma 3.3 and the fact that $|\varphi|_{\operatorname{Lip}^{0}\left(H_{1}, \mathbb{R}\right)}<\infty$ establish that

$$
\left([0, T] \times H_{1} \ni(t, x) \mapsto u(t, x) \in \mathbb{R}\right) \in C\left([0, T] \times H_{1}, \mathbb{R}\right)
$$


Moreover, item (可) of Lemma 3.3 proves that

$$
u_{1,0} \in C\left([0, T] \times H_{1}, \mathbb{R}\right) .
$$

It is well known that $u$ is a strong solution of the Kolmogorov equation associated to $X^{\mathbb{H}, x}:[0, T] \times \Omega \rightarrow H, x \in H$. More precisely, note that for all $t \in(0, T), x \in H_{1}$ it holds that

$$
u_{1,0}(t, x)=-u_{0,1}(t, x)(A x+F(x))-\frac{1}{2} \sum_{b \in \mathbb{U}} u_{0,2}(t, x)\left(B^{b}(x), B^{b}(x)\right)
$$

(cf., e.g., Da Prato \& Zabczyk [16, Theorem 9.25]). In addition, observe that for all $k \in\{1,2\}$ it holds that the function

$$
\begin{array}{r}
\left([0, T] \times H_{1} \ni(t, x) \mapsto\left(\left(H_{1}\right)^{k} \ni\left(h_{1}, \ldots, h_{k}\right) \mapsto u_{0, k}(t, x)\left(h_{1}, \ldots, h_{k}\right) \in \mathbb{R}\right)\right. \\
\left.\in L^{(k)}\left(H_{1}, \mathbb{R}\right)\right)
\end{array}
$$

is continuous (cf., e.g., Da Prato \& Zabczyk [16, Theorem 9.25] and Andersson et al. 11, Theorem 3.3]). Combining (164), (165), and Corollary 3.6 with the standard Itô formula in infinite dimensions (cf., e.g., Brzeźniak et al. [9, Theorem 2.4]) hence proves that it holds $\mathbb{P}$-a.s. that

$$
\begin{aligned}
u\left(T, Y_{T}^{I}\right)-u\left(0, Y_{0}^{I}\right) & \\
= & \int_{0}^{T} u_{1,0}\left(t, Y_{t}^{I}\right)+u_{0,1}\left(t, Y_{t}^{I}\right)\left(A Y_{t}^{I}+F_{I}\left(Y_{t}^{I}\right)\right) d t+\int_{0}^{T} u_{0,1}\left(t, Y_{t}^{I}\right) B_{I}\left(Y_{t}^{I}\right) d W_{t} \\
& +\frac{1}{2} \int_{0}^{T} \sum_{b \in \mathbb{U}} u_{0,2}\left(t, Y_{t}^{I}\right)\left(B_{I}^{b}\left(Y_{t}^{I}\right), B_{I}^{b}\left(Y_{t}^{I}\right)\right) d t .
\end{aligned}
$$

This and (166) ensure that it holds $\mathbb{P}$-a.s. that

$$
\begin{aligned}
u\left(T, Y_{T}^{I}\right)-u\left(0, Y_{0}^{I}\right) & \\
= & \int_{0}^{T} u_{0,1}\left(t, Y_{t}^{I}\right)\left(F_{I}\left(Y_{t}^{I}\right)-F\left(Y_{t}^{I}\right)\right) d t+\int_{0}^{T} u_{0,1}\left(t, Y_{t}^{I}\right) B_{I}\left(Y_{t}^{I}\right) d W_{t} \\
& +\frac{1}{2} \int_{0}^{T} \sum_{b \in \mathbb{U}} u_{0,2}\left(t, Y_{t}^{I}\right)\left(B_{I}^{b}\left(Y_{t}^{I}\right)+B^{b}\left(Y_{t}^{I}\right), B_{I}^{b}\left(Y_{t}^{I}\right)-B^{b}\left(Y_{t}^{I}\right)\right) d t .
\end{aligned}
$$

Next note that Lemma 3.4 shows that

$$
c_{0}+c_{0,0}+c_{0,0,0}<\infty
$$

This guarantees that

$$
\begin{aligned}
\sup _{t \in[0, T)} \sup _{x \in H} \frac{\left\|u_{0,1}(t, x) B_{I}(x)\right\|_{H S(U, \mathbb{R})}}{g_{1}(x)} & \leq \sup _{t \in[0, T)} \sup _{x \in H}\left[\frac{\left\|u_{0,1}(t, x)\right\|_{L(H, \mathbb{R})}\left\|B_{I}(x)\right\|_{H S(U, H)}}{g_{1}(x)}\right] \\
& \leq c_{0}\left[\sup _{t \in[0, T)} \sup _{x \in H} \frac{\left\|B_{I}(x)\right\|_{H S(U, H)}}{g_{1}(x)}\right]<\infty .
\end{aligned}
$$

This and the fact that $K_{2}^{I}<\infty$ assure that

$$
\mathbb{E}\left[\int_{0}^{T}\left\|u_{0,1}\left(t, Y_{t}^{I}\right) B_{I}\left(Y_{t}^{I}\right)\right\|_{H S(U, \mathbb{R})}^{2} d t\right]<\infty .
$$

Furthermore, note that Markov's property associated to $X^{\mathbb{H}, x}:[0, T] \times \Omega \rightarrow H, x \in H$, implies that

$$
\mathbb{E}\left[\varphi\left(Y_{T}^{I}\right)-\varphi\left(X_{T}^{\mathbb{H}}\right)\right]=\mathbb{E}\left[u\left(T, Y_{T}^{I}\right)-u\left(0, Y_{0}^{I}\right)\right]
$$


(cf., e.g., Theorem 9.14 in Da Prato \& Zabczyk [16]). Combining (169) with (172) therefore shows that $\mathbb{E}\left[\int_{0}^{T} u_{0,1}\left(t, Y_{t}^{I}\right) B_{I}\left(Y_{t}^{I}\right) d W_{t}\right]=0$ and

$$
\begin{aligned}
\mathbb{E} & {\left[\varphi\left(Y_{T}^{I}\right)-\varphi\left(X_{T}^{\mathbb{H}}\right)\right]=\mathbb{E}\left[u\left(T, Y_{T}^{I}\right)-u\left(0, Y_{0}^{I}\right)\right] } \\
= & \int_{0}^{T} \mathbb{E}\left[u_{0,1}\left(t, Y_{t}^{I}\right)\left(F_{I}\left(Y_{t}^{I}\right)-F\left(Y_{t}^{I}\right)\right)\right] d t \\
& +\frac{1}{2} \sum_{b \in \mathbb{U}} \int_{0}^{T} \mathbb{E}\left[u_{0,2}\left(t, Y_{t}^{I}\right)\left(B_{I}^{b}\left(Y_{t}^{I}\right)+B^{b}\left(Y_{t}^{I}\right), B_{I}^{b}\left(Y_{t}^{I}\right)-B^{b}\left(Y_{t}^{I}\right)\right)\right] d t .
\end{aligned}
$$

The identities in (174) provide us an explicit representation for the weak approximation error $\mathbb{E}\left[\varphi\left(Y_{T}^{I}\right)\right]-\mathbb{E}\left[\varphi\left(X_{T}^{\mathbb{H}}\right)\right]$. In the following we employ the mild Itô formula in Corollary 2 in Da Prato et al. 14 to estimate the two summands appearing on the right hand side of (174). We first concentrate on the first summand on the right hand side of (174). For the application of the mild Itô formula in [14, Corollary 2] to the first summand on the right hand side of (174) (see (177) below) it is convenient to introduce suitable auxiliary functions. More formally, let $\tilde{F}_{t, s}: H \rightarrow \mathbb{R}, t \in(s, T], s \in[0, T)$, be the functions which satisfy for all $s \in[0, T), t \in(s, T], x \in H$ that

$$
\begin{aligned}
& \tilde{F}_{t, s}(x) \\
& =u_{0,2}\left(t, e^{A(t-s)} x\right)\left(F_{I}\left(e^{A(t-s)} x\right)-F\left(e^{A(t-s)} x\right), e^{A(t-s)} F_{I}(x)\right) \\
& +u_{0,1}\left(t, e^{A(t-s)} x\right)\left(\left[F_{I}^{\prime}\left(e^{A(t-s)} x\right)-F^{\prime}\left(e^{A(t-s)} x\right)\right] e^{A(t-s)} F_{I}(x)\right) \\
& +\frac{1}{2} \sum_{b \in \mathbb{U}} u_{0,3}\left(t, e^{A(t-s)} x\right)\left(F_{I}\left(e^{A(t-s)} x\right)-F\left(e^{A(t-s)} x\right), e^{A(t-s)} B_{I}^{b}(x), e^{A(t-s)} B_{I}^{b}(x)\right) \\
& +\sum_{b \in \mathbb{U}} u_{0,2}\left(t, e^{A(t-s)} x\right)\left(\left[F_{I}^{\prime}\left(e^{A(t-s)} x\right)-F^{\prime}\left(e^{A(t-s)} x\right)\right] e^{A(t-s)} B_{I}^{b}(x), e^{A(t-s)} B_{I}^{b}(x)\right) \\
& +\frac{1}{2} \sum_{b \in \mathbb{U}} u_{0,1}\left(t, e^{A(t-s)} x\right)\left(\left[F_{I}^{\prime \prime}\left(e^{A(t-s)} x\right)-F^{\prime \prime}\left(e^{A(t-s)} x\right)\right]\left(e^{A(t-s)} B_{I}^{b}(x), e^{A(t-s)} B_{I}^{b}(x)\right)\right)
\end{aligned}
$$

and let $\hat{F}_{t, s}: H \rightarrow H S(U, \mathbb{R}), t \in(s, T], s \in[0, T)$, be the functions which satisfy for all $s \in[0, T), t \in(s, T], x \in H$ that

$$
\begin{aligned}
& \hat{F}_{t, s}(x) \\
& =u_{0,2}\left(t, e^{A(t-s)} x\right)\left(F_{I}\left(e^{A(t-s)} x\right)-F\left(e^{A(t-s)} x\right), e^{A(t-s)} B_{I}(x)\right) \\
& +u_{0,1}\left(t, e^{A(t-s)} x\right)\left(\left[F_{I}^{\prime}\left(e^{A(t-s)} x\right)-F^{\prime}\left(e^{A(t-s)} x\right)\right] e^{A(t-s)} B_{I}(x)\right) .
\end{aligned}
$$

An application of the mild Itô formula in Corollary 2 in Da Prato et al. [14] then proves that for all $t \in(0, T]$ it holds $\mathbb{P}$-a.s. that

$$
\begin{aligned}
& u_{0,1}\left(t, Y_{t}^{I}\right)\left(F_{I}\left(Y_{t}^{I}\right)-F\left(Y_{t}^{I}\right)\right) \\
& =u_{0,1}\left(t, e^{A t} \xi\right)\left(F_{I}\left(e^{A t} \xi\right)-F\left(e^{A t} \xi\right)\right)+\int_{0}^{t} \tilde{F}_{t, s}\left(Y_{s}^{I}\right) d s+\int_{0}^{t} \hat{F}_{t, s}\left(Y_{s}^{I}\right) d W_{s} .
\end{aligned}
$$

Moreover, observe that (170), (176), and Lemma 1.2 ensure that

$$
\sup _{s \in[0, T)} \sup _{t \in(s, T)} \sup _{x \in H} \frac{\left\|\hat{F}_{t, s}(x)\right\|_{H S(U, \mathbb{R})}}{g_{2}(x)}<\infty .
$$

This and the fact that $K_{I}^{4}<\infty$ assure that for all $t \in(0, T)$ it holds that

$$
\mathbb{E}\left[\int_{0}^{t}\left\|\hat{F}_{t, s}\left(Y_{s}^{I}\right)\right\|_{H S(U, \mathbb{R})}^{2} d s\right]<\infty .
$$


This and (177) yield that for all $t \in(0, T)$ it holds that $\mathbb{E}\left[\left|\int_{0}^{t} \hat{F}_{t, s}\left(Y_{s}^{I}\right) d W_{s}\right|\right]<\infty$, $\mathbb{E}\left[\int_{0}^{t} \hat{F}_{t, s}\left(Y_{s}^{I}\right) d W_{s}\right]=0$, and

$$
\begin{aligned}
& \mathbb{E}\left[u_{0,1}\left(t, Y_{t}^{I}\right)\left(F_{I}\left(Y_{t}^{I}\right)-F\left(Y_{t}^{I}\right)\right)\right] \\
& =\mathbb{E}\left[u_{0,1}\left(t, e^{A t} \xi\right)\left(F_{I}\left(e^{A t} \xi\right)-F\left(e^{A t} \xi\right)\right)\right]+\int_{0}^{t} \mathbb{E}\left[\tilde{F}_{t, s}\left(Y_{s}^{I}\right)\right] d s .
\end{aligned}
$$

Next we apply the mild Itô formula in [14, Corollary 2] to the second summand on the right hand side of (174). For this application it is again convenient to introduce suitable functions. More formally, let $\tilde{B}_{t, s}: H \rightarrow \mathbb{R}, t \in(s, T], s \in[0, T)$, be the functions which satisfy for all $s \in[0, T), t \in(s, T], x \in H$ that

$$
\begin{aligned}
& \tilde{B}_{t, s}(x) \\
& =\sum_{b \in \mathbb{U}} u_{0,2}\left(t, e^{A(t-s)} x\right)\left(\left[\left(B_{I}^{b}\right)^{\prime}\left(e^{A(t-s)} x\right)+\left(B^{b}\right)^{\prime}\left(e^{A(t-s)} x\right)\right] e^{A(t-s)} F_{I}(x),\right. \\
& \left.B_{I}^{b}\left(e^{A(t-s)} x\right)-B^{b}\left(e^{A(t-s)} x\right)\right) \\
& +\sum_{b \in \mathbb{U}} u_{0,2}\left(t, e^{A(t-s)} x\right)\left(B_{I}^{b}\left(e^{A(t-s)} x\right)+B^{b}\left(e^{A(t-s)} x\right),\right. \\
& \left.\left[\left(B_{I}^{b}\right)^{\prime}\left(e^{A(t-s)} x\right)-\left(B^{b}\right)^{\prime}\left(e^{A(t-s)} x\right)\right] e^{A(t-s)} F_{I}(x)\right) \\
& +\sum_{b \in \mathbb{U}} u_{0,3}\left(t, e^{A(t-s)} x\right)\left(B_{I}^{b}\left(e^{A(t-s)} x\right)+B^{b}\left(e^{A(t-s)} x\right)\right. \\
& \left.B_{I}^{b}\left(e^{A(t-s)} x\right)-B^{b}\left(e^{A(t-s)} x\right), e^{A(t-s)} F_{I}(x)\right) \\
& +\sum_{b_{1}, b_{2} \in \mathbb{U}} u_{0,2}\left(t, e^{A(t-s)} x\right)\left(\left[\left(B_{I}^{b_{2}}\right)^{\prime}\left(e^{A(t-s)} x\right)+\left(B^{b_{2}}\right)^{\prime}\left(e^{A(t-s)} x\right)\right] e^{A(t-s)} B_{I}^{b_{1}}(x),\right. \\
& \left.\left[\left(B_{I}^{b_{2}}\right)^{\prime}\left(e^{A(t-s)} x\right)-\left(B^{b_{2}}\right)^{\prime}\left(e^{A(t-s)} x\right)\right] e^{A(t-s)} B_{I}^{b_{1}}(x)\right) \\
& +\frac{1}{2} \sum_{b_{1}, b_{2} \in \mathbb{U}} u_{0,2}\left(t, e^{A(t-s)} x\right)\left(B_{I}^{b_{2}}\left(e^{A(t-s)} x\right)-B^{b_{2}}\left(e^{A(t-s)} x\right)\right. \\
& \left.\left[\left(B_{I}^{b_{2}}\right)^{\prime \prime}\left(e^{A(t-s)} x\right)+\left(B^{b_{2}}\right)^{\prime \prime}\left(e^{A(t-s)} x\right)\right]\left(e^{A(t-s)} B_{I}^{b_{1}}(x), e^{A(t-s)} B_{I}^{b_{1}}(x)\right)\right) \\
& +\frac{1}{2} \sum_{b_{1}, b_{2} \in \mathbb{U}} u_{0,2}\left(t, e^{A(t-s)} x\right)\left(B_{I}^{b_{2}}\left(e^{A(t-s)} x\right)+B^{b_{2}}\left(e^{A(t-s)} x\right)\right. \\
& \left.\left[\left(B_{I}^{b_{2}}\right)^{\prime \prime}\left(e^{A(t-s)} x\right)-\left(B^{b_{2}}\right)^{\prime \prime}\left(e^{A(t-s)} x\right)\right]\left(e^{A(t-s)} B_{I}^{b_{1}}(x), e^{A(t-s)} B_{I}^{b_{1}}(x)\right)\right) \\
& +\sum_{b_{1}, b_{2} \in \mathbb{U}} u_{0,3}\left(t, e^{A(t-s)} x\right)\left(\left[\left(B_{I}^{b_{2}}\right)^{\prime}\left(e^{A(t-s)} x\right)+\left(B^{b_{2}}\right)^{\prime}\left(e^{A(t-s)} x\right)\right] e^{A(t-s)} B_{I}^{b_{1}}(x),\right. \\
& \left.B_{I}^{b_{2}}\left(e^{A(t-s)} x\right)-B^{b_{2}}\left(e^{A(t-s)} x\right), e^{A(t-s)} B_{I}^{b_{1}}(x)\right) \\
& +\sum_{b_{1}, b_{2} \in \mathbb{U}} u_{0,3}\left(t, e^{A(t-s)} x\right)\left(B_{I}^{b_{2}}\left(e^{A(t-s)} x\right)+B^{b_{2}}\left(e^{A(t-s)} x\right),\right. \\
& \left.\left[\left(B_{I}^{b_{2}}\right)^{\prime}\left(e^{A(t-s)} x\right)-\left(B^{b_{2}}\right)^{\prime}\left(e^{A(t-s)} x\right)\right] e^{A(t-s)} B_{I}^{b_{1}}(x), e^{A(t-s)} B_{I}^{b_{1}}(x)\right) \\
& +\frac{1}{2} \sum_{b_{1}, b_{2} \in \mathbb{U}} u_{0,4}\left(t, e^{A(t-s)} x\right)\left(B_{I}^{b_{2}}\left(e^{A(t-s)} x\right)+B^{b_{2}}\left(e^{A(t-s)} x\right), B_{I}^{b_{2}}\left(e^{A(t-s)} x\right)-B^{b_{2}}\left(e^{A(t-s)} x\right),\right. \\
& \left.e^{A(t-s)} B_{I}^{b_{1}}(x), e^{A(t-s)} B_{I}^{b_{1}}(x)\right)
\end{aligned}
$$

and let $\hat{B}_{t, s}: H \rightarrow H S(U, \mathbb{R}), t \in(s, T], s \in[0, T)$, be the functions which satisfy for all 
$s \in[0, T), t \in(s, T], x \in H$ that

$$
\begin{gathered}
\hat{B}_{t, s}(x) \\
=\sum_{b \in \mathbb{U}} u_{0,2}\left(t, e^{A(t-s)} x\right)\left(\left[\left(B_{I}^{b}\right)^{\prime}\left(e^{A(t-s)} x\right)+\left(B^{b}\right)^{\prime}\left(e^{A(t-s)} x\right)\right] e^{A(t-s)} B_{I}(x),\right. \\
\left.B_{I}^{b}\left(e^{A(t-s)} x\right)-B^{b}\left(e^{A(t-s)} x\right)\right) \\
+\sum_{b \in \mathbb{U}} u_{0,2}\left(t, e^{A(t-s)} x\right)\left(B_{I}^{b}\left(e^{A(t-s)} x\right)+B^{b}\left(e^{A(t-s)} x\right),\right. \\
\left.\left[\left(B_{I}^{b}\right)^{\prime}\left(e^{A(t-s)} x\right)-\left(B^{b}\right)^{\prime}\left(e^{A(t-s)} x\right)\right] e^{A(t-s)} B_{I}(x)\right) \\
+\sum_{b \in \mathbb{U}} u_{0,3}\left(t, e^{A(t-s)} x\right)\left(B_{I}^{b}\left(e^{A(t-s)} x\right)+B^{b}\left(e^{A(t-s)} x\right),\right. \\
\left.B_{I}^{b}\left(e^{A(t-s)} x\right)-B^{b}\left(e^{A(t-s)} x\right), e^{A(t-s)} B_{I}(x)\right) .
\end{gathered}
$$

An application of the mild Itô formula in Corollary 2 in Da Prato et al. [14 then proves that for all $t \in(0, T]$ it holds $\mathbb{P}$-a.s. that

$$
\begin{aligned}
& \sum_{b \in \mathbb{U}} u_{0,2}\left(t, Y_{t}^{I}\right)\left(B_{I}^{b}\left(Y_{t}^{I}\right)+B^{b}\left(Y_{t}^{I}\right), B_{I}^{b}\left(Y_{t}^{I}\right)-B^{b}\left(Y_{t}^{I}\right)\right) \\
& =\sum_{b \in \mathbb{U}} u_{0,2}\left(t, e^{A t} \xi\right)\left(B_{I}^{b}\left(e^{A t} \xi\right)+B^{b}\left(e^{A t} \xi\right), B_{I}^{b}\left(e^{A t} \xi\right)-B^{b}\left(e^{A t} \xi\right)\right) \\
& \quad+\int_{0}^{t} \tilde{B}_{t, s}\left(Y_{s}^{I}\right) d s+\int_{0}^{t} \hat{B}_{t, s}\left(Y_{s}^{I}\right) d W_{s} .
\end{aligned}
$$

Next observe that there exists a continuous $\left(\mathcal{F}_{t}\right)_{t \in[0, T]}$-adapted stochastic process $\mathcal{Y}:[0, T] \times$ $\Omega \rightarrow H$ which satisfies for all $t \in[0, T]$ that $\mathbb{P}\left(\mathcal{Y}_{t}=Y_{t}^{I}\right)=1$ and

$$
\mathbb{E}\left[\sup _{s \in[0, T]}\left\|\mathcal{Y}_{s}\right\|_{H}^{4}\right]<\infty
$$

(cf., e.g., Da Prato \& Zabczyk [16, Theorem 7.2]). This and, e.g., Lemma 3.1 in Jentzen \& Pušnik [30] ensure that for all $t \in(0, T]$ it holds $\mathbb{P}$-a.s. that

$$
\int_{0}^{t}\left\|\hat{B}_{t, s}\left(Y_{s}^{I}\right)\right\|_{H S(U, \mathbb{R})}^{2} d s=\int_{0}^{t}\left\|\hat{B}_{t, s}\left(\mathcal{Y}_{s}\right)\right\|_{H S(U, \mathbb{R})}^{2} d s .
$$

Moreover, note that (170), (182), and Lemma 1.2 assure that

$$
\sup _{s \in[0, T)} \sup _{t \in(s, T)} \sup _{x \in H} \frac{\left\|\hat{B}_{t, s}(x)\right\|_{H S(U, \mathbb{R})}}{g_{3}(x)}<\infty .
$$

Combining (184)-(186) yields that for all $t \in(0, T)$ it holds that

$$
\begin{aligned}
& \mathbb{E}\left[\left(\int_{0}^{t}\left\|\hat{B}_{t, s}\left(Y_{s}^{I}\right)\right\|_{H S(U, \mathbb{R})}^{2} d s\right)^{1 / 2}\right] \\
& =\mathbb{E}\left[\left(\int_{0}^{t}\left\|\hat{B}_{t, s}\left(\mathcal{Y}_{s}\right)\right\|_{H S(U, \mathbb{R})}^{2} d s\right)^{1 / 2}\right] \\
& \leq\left[\sup _{s \in[0, t)} \sup _{x \in H} \frac{\left\|\hat{B}_{t, s}(x)\right\|_{H S(U, \mathbb{R})}}{g_{3}(x)}\right] \mathbb{E}\left[\left(\int_{0}^{t} g_{6}\left(\mathcal{Y}_{s}\right) d s\right)^{1 / 2}\right] \\
& \leq \sqrt{t}\left[\sup _{s \in[0, t)} \sup _{x \in H} \frac{\left\|\hat{B}_{t, s}(x)\right\|_{H S(U, \mathbb{R})}}{g_{3}(x)}\right] \mathbb{E}\left[\sup _{s \in[0, t]} \max \left\{1,\left\|\mathcal{Y}_{s}\right\|_{H}^{3}\right\}\right]<\infty .
\end{aligned}
$$


This shows that for all $t \in(0, T)$ it holds that $\mathbb{E}\left[\left|\int_{0}^{t} \hat{B}_{t, s}\left(Y_{s}^{I}\right) d W_{s}\right|\right]<\infty$ and

$$
\mathbb{E}\left[\int_{0}^{t} \hat{B}_{t, s}\left(Y_{s}^{I}\right) d W_{s}\right]=0
$$

(cf., e.g., van Neerven et al. [46, Theorem 4.7]). This and (183) ensure that for all $t \in(0, T)$ it holds that

$$
\begin{aligned}
& \sum_{b \in \mathbb{U}} \mathbb{E}\left[u_{0,2}\left(t, Y_{t}^{I}\right)\left(B_{I}^{b}\left(Y_{t}^{I}\right)+B^{b}\left(Y_{t}^{I}\right), B_{I}^{b}\left(Y_{t}^{I}\right)-B^{b}\left(Y_{t}^{I}\right)\right)\right] \\
& =\sum_{b \in \mathbb{U}} \mathbb{E}\left[u_{0,2}\left(t, e^{A t} \xi\right)\left(B_{I}^{b}\left(e^{A t} \xi\right)+B^{b}\left(e^{A t} \xi\right), B_{I}^{b}\left(e^{A t} \xi\right)-B^{b}\left(e^{A t} \xi\right)\right)\right] \\
& \quad+\int_{0}^{t} \mathbb{E}\left[\tilde{B}_{t, s}\left(Y_{s}^{I}\right)\right] d s .
\end{aligned}
$$

Putting (180) and (189) into (174) proves that

$$
\begin{aligned}
& \mathbb{E}\left[\varphi\left(Y_{T}^{I}\right)\right]-\mathbb{E}\left[\varphi\left(X_{T}^{\mathbb{H}}\right)\right] \\
& =\int_{0}^{T} \mathbb{E}\left[u_{0,1}\left(t, e^{A t} \xi\right)\left(F_{I}\left(e^{A t} \xi\right)-F\left(e^{A t} \xi\right)\right)\right] d t \\
& +\frac{1}{2} \sum_{b \in \mathbb{U}} \int_{0}^{T} \mathbb{E}\left[u_{0,2}\left(t, e^{A t} \xi\right)\left(B_{I}^{b}\left(e^{A t} \xi\right)+B^{b}\left(e^{A t} \xi\right), B_{I}^{b}\left(e^{A t} \xi\right)-B^{b}\left(e^{A t} \xi\right)\right)\right] d t \\
& +\int_{0}^{T} \int_{0}^{t} \mathbb{E}\left[\tilde{F}_{t, s}\left(Y_{s}^{I}\right)\right]+\frac{1}{2} \mathbb{E}\left[\tilde{B}_{t, s}\left(Y_{s}^{I}\right)\right] d s d t .
\end{aligned}
$$

In the following we estimate the absolute values of the summands on the right hand side of (190). To this end we observe that Lemma 3.4 and the hypothesis that $\vartheta<1 / 2$ demonstrate that $c_{-\vartheta}<\infty$. This and Lemma 1.2 ensure that for all $t \in(0, T)$ it holds that

$$
\begin{aligned}
& \left|u_{0,1}\left(t, e^{A t} \xi\right)\left(F_{I}\left(e^{A t} \xi\right)-F\left(e^{A t} \xi\right)\right)\right| \leq \frac{c_{-\vartheta}}{(T-t)^{\vartheta}}\left\|F_{I}\left(e^{A t} \xi\right)-F\left(e^{A t} \xi\right)\right\|_{H_{-\vartheta}} \\
& \leq \frac{c_{-\vartheta}|F|_{C_{b}^{1}\left(H, H_{-\vartheta}\right)}\left\|P_{\mathbb{H} \backslash I}\right\|_{L\left(H, H_{-\rho}\right)}\|\xi\|_{H}}{(T-t)^{\vartheta} t^{\rho}} .
\end{aligned}
$$

Next note that Hölder's inequality implies that for all $x, y \in(0, \infty)$ with $(x-1)(y-1) \geq 0$ and $x+y>1$ it holds that

$$
\int_{0}^{1}(1-t)^{(x-1)} t^{(y-1)} d t \leq \frac{1}{(x+y-1)} .
$$

This, the fact that $\mathbb{E}\left[\|\xi\|_{H}\right] \leq K_{1}^{I}$, and (191) imply that

$$
\begin{aligned}
& \left|\int_{0}^{T} \mathbb{E}\left[u_{0,1}\left(t, e^{A t} \xi\right)\left(F_{I}\left(e^{A t} \xi\right)-F\left(e^{A t} \xi\right)\right)\right] d t\right| \\
& \leq \frac{\left.K_{1}^{I} c_{-\vartheta} T^{(1-\vartheta-\rho)}|F|_{C_{b}^{1}\left(H, H_{-\vartheta}\right)}|| P_{\mathbb{H} \backslash I}\right|_{L\left(H, H_{-\rho}\right)}}{(1-\vartheta-\rho)} .
\end{aligned}
$$

Inequality (193) provides us an estimate for the absolute value of the first summand on the right hand side of (190). In the next step we bound the absolute value of the second 
summand on the right hand side (190). For this we observe that the Cauchy-Schwarz inequality ensures that for all $t \in[0, T)$ it holds that

$$
\begin{aligned}
\sum_{b \in \mathbb{U}}\left|u_{0,2}\left(t, e^{A t} \xi\right)\left(B_{I}^{b}\left(e^{A t} \xi\right)+B^{b}\left(e^{A t} \xi\right), B_{I}^{b}\left(e^{A t} \xi\right)-B^{b}\left(e^{A t} \xi\right)\right)\right| \\
\leq \frac{c_{-\vartheta / 2,-\vartheta / 2}}{(T-t)^{\vartheta}}\left[\sum_{b \in \mathbb{U}}\left\|B_{I}^{b}\left(e^{A t} \xi\right)+B^{b}\left(e^{A t} \xi\right)\right\|_{H_{-\vartheta / 2}}\left\|B_{I}^{b}\left(e^{A t} \xi\right)-B^{b}\left(e^{A t} \xi\right)\right\|_{H_{-\vartheta / 2}}\right] \\
\leq \frac{c_{-\vartheta / 2,-\vartheta / 2}}{(T-t)^{\vartheta}}\left[\sum_{b \in \mathbb{U}}\left\|B_{I}^{b}\left(e^{A t} \xi\right)+B^{b}\left(e^{A t} \xi\right)\right\|_{H_{-\vartheta / 2}}^{2}\right]^{1 / 2} \\
\\
\cdot\left[\sum_{b \in \mathbb{U}}\left\|B_{I}^{b}\left(e^{A t} \xi\right)-B^{b}\left(e^{A t} \xi\right)\right\|_{H_{-\vartheta / 2}}^{2}\right]^{1 / 2} \\
=\frac{c_{-\vartheta / 2,-\vartheta / 2}}{(T-t)^{\vartheta}}\left\|B_{I}\left(e^{A t} \xi\right)+B\left(e^{A t} \xi\right)\right\|_{H S\left(U, H_{-\vartheta / 2}\right)}\left\|B_{I}\left(e^{A t} \xi\right)-B\left(e^{A t} \xi\right)\right\|_{H S\left(U, H_{-\vartheta / 2}\right.} .
\end{aligned}
$$

The fact that $g_{1}(\xi)\|\xi\|_{H} \leq g_{2}(\xi)$ hence shows that for all $t \in(0, T)$ it holds that

$$
\begin{aligned}
& \sum_{b \in \mathbb{U}}\left|u_{0,2}\left(t, e^{A t} \xi\right)\left(B_{I}^{b}\left(e^{A t} \xi\right)+B^{b}\left(e^{A t} \xi\right), B_{I}^{b}\left(e^{A t} \xi\right)-B^{b}\left(e^{A t} \xi\right)\right)\right| \\
& \leq \frac{c_{-\vartheta / 2,-\vartheta / 2}}{(T-t)^{\vartheta}}\left[\left\|B\left(P_{I} e^{A t} \xi\right)\right\|_{H S\left(U, H_{-\vartheta / 2}\right)}+\left\|B\left(e^{A t} \xi\right)\right\|_{H S\left(U, H_{-} / 2\right.}\right) \\
& \cdot\|B\|_{C_{b}^{1}\left(H, H S\left(U, H_{-\vartheta / 2}\right)\right)}\left\|P_{\mathbb{H} \backslash I} e^{A t} \xi\right\|_{H} \\
& \leq \frac{c_{-\vartheta / 2,-\vartheta / 2}}{(T-t)^{\vartheta}}\|B\|_{C_{b}^{1}\left(H, H S\left(U, H_{-\vartheta / 2}\right)\right)}^{2}\left(g_{1}\left(P_{I} e^{A t} \xi\right)+g_{1}\left(e^{A t} \xi\right)\right)\left\|P_{\mathbb{H} \backslash I} e^{A t} \xi\right\|_{H} \\
& \leq \frac{2 c_{-\vartheta / 2,-\vartheta / 2} g_{2}(\xi)}{(T-t)^{\vartheta} t^{\rho}}\|B\|_{C_{b}^{1}\left(H, H S\left(U, H_{-\vartheta / 2}\right)\right)}^{2}\left\|P_{\mathbb{H} \backslash I}\right\|_{L\left(H, H_{-\rho}\right)} .
\end{aligned}
$$

This, the fact that $\mathbb{E}\left[g_{2}(\xi)\right] \leq K_{2}^{I}$, and (192) imply that

$$
\begin{aligned}
& \left|\sum_{b \in \mathbb{U}} \int_{0}^{T} \mathbb{E}\left[u_{0,2}\left(t, e^{A t} \xi\right)\left(B_{I}^{b}\left(e^{A t} \xi\right)+B^{b}\left(e^{A t} \xi\right), B_{I}^{b}\left(e^{A t} \xi\right)-B^{b}\left(e^{A t} \xi\right)\right)\right] d t\right| \\
& \leq \frac{2 K_{2}^{I} c_{-\vartheta / 2,-\vartheta / 2} T^{(1-\vartheta-\rho)}}{(1-\vartheta-\rho)}\|B\|_{C_{b}^{1}\left(H, H S\left(U, H_{-\vartheta / 2}\right)\right)}^{2}\left\|P_{\mathbb{H} \backslash I}\right\|_{L\left(H, H_{-\rho}\right)} .
\end{aligned}
$$

Inequality (196) provides us an estimate for the second term on the right hand side of (190). In the next step we bound the absolute value of the term $\int_{0}^{T} \int_{0}^{t} \mathbb{E}\left[\tilde{F}_{t, s}\left(Y_{s}^{I}\right)\right] d s d t$ on the right hand side of (190). For this we note that (175) shows that for all $s, t \in[0, T)$, $x \in H$ with $t>s$ it holds that

$$
\begin{aligned}
& \left|\tilde{F}_{t, s}(x)\right| \\
& \leq \frac{c_{-\vartheta, 0}}{(T-t)^{\vartheta}}\left\|F_{I}\left(e^{A(t-s)} x\right)-F\left(e^{A(t-s)} x\right)\right\|_{H_{-\vartheta}}\left\|e^{A(t-s)} F_{I}(x)\right\|_{H} \\
& +\frac{c_{-\vartheta}}{(T-t)^{\vartheta}}\left\|\left[F_{I}^{\prime}\left(e^{A(t-s)} x\right)-F^{\prime}\left(e^{A(t-s)} x\right)\right] e^{A(t-s)} F_{I}(x)\right\|_{H_{-\vartheta}} \\
& +\frac{c_{-\vartheta, 0,0}}{2(T-t)^{\vartheta}}\left\|F_{I}\left(e^{A(t-s)} x\right)-F\left(e^{A(t-s)} x\right)\right\|_{H_{-\vartheta}}\left\|e^{A(t-s)} B_{I}(x)\right\|_{H S(U, H)}^{2} \\
& +\frac{c_{-\vartheta, 0}}{(T-t)^{\vartheta}} \sum_{b \in \mathbb{U}}\left[\left\|\left[F_{I}^{\prime}\left(e^{A(t-s)} x\right)-F^{\prime}\left(e^{A(t-s)} x\right)\right] e^{A(t-s)} B_{I}^{b}(x)\right\|_{H_{-\vartheta}}\left\|e^{A(t-s)} B_{I}^{b}(x)\right\|_{H}\right] \\
& +\frac{c_{-\vartheta}}{2(T-t)^{\vartheta}} \sum_{b \in \mathbb{U}}\left\|\left[F_{I}^{\prime \prime}\left(e^{A(t-s)} x\right)-F^{\prime \prime}\left(e^{A(t-s)} x\right)\right]\left(e^{A(t-s)} B_{I}^{b}(x), e^{A(t-s)} B_{I}^{b}(x)\right)\right\|_{H_{-\vartheta}} .
\end{aligned}
$$


Next observe that for all $x, v \in H, r \in[0, \vartheta], s, t \in[0, T]$ with $s<t$ it holds that

$$
\begin{aligned}
\| & {\left[F_{I}^{\prime}\left(e^{A(t-s)} x\right)-F^{\prime}\left(e^{A(t-s)} x\right)\right] e^{A(t-s)} v \|_{H_{-\vartheta}} } \\
= & \left\|\left[F^{\prime}\left(e^{A(t-s)} P_{I}(x)\right) P_{I}-F^{\prime}\left(e^{A(t-s)} x\right)\right] e^{A(t-s)} v\right\|_{H_{-\vartheta}} \\
\leq & \left\|\left[F^{\prime}\left(e^{A(t-s)} P_{I}(x)\right)-F^{\prime}\left(e^{A(t-s)} x\right)\right] P_{I} e^{A(t-s)} v\right\|_{H_{-\vartheta}} \\
& +\left\|F^{\prime}\left(e^{A(t-s)} x\right) P_{\mathbb{H} \backslash I} e^{A(t-s)} v\right\|_{H_{-\vartheta}} \\
\leq & |F|_{C_{b}^{2}\left(H, H_{-\vartheta}\right)}\left\|e^{A(t-s)} P_{\mathbb{H} \backslash I} x\right\|_{H}\left\|P_{I} e^{A(t-s)} v\right\|_{H}+\|F\|_{C_{b}^{1}\left(H, H_{-\vartheta}\right)}\left\|P_{\mathbb{H} \backslash I} e^{A(t-s)} v\right\|_{H} \\
\leq & {\left[g_{1}(x)|F|_{C_{b}^{2}\left(H, H_{-\vartheta}\right)}+\|F\|_{C_{b}^{1}\left(H, H_{-\vartheta}\right)}\right] \frac{\|v\|_{H_{-r}}\left\|P_{\mathbb{H} \backslash I}\right\|_{L\left(H, H_{-\rho}\right)}}{(t-s)^{\rho+r}} } \\
\leq & \frac{g_{1}(x)\|F\|_{C_{b}^{2}\left(H, H_{-\vartheta}\right)}\|v\|_{H_{-r}}\left\|P_{\mathbb{H} \backslash I}\right\|_{L\left(H, H_{-\rho}\right)} .}{(t-s)^{(\rho+r)}}
\end{aligned}
$$

This and the fact that for all $x \in H$ it holds that $g_{1}(x)\|x\|_{H} \leq g_{2}(x)$ imply that for all $x \in H, s, t \in[0, T]$ with $s<t$ it holds that

$$
\begin{aligned}
\|\left[F_{I}^{\prime}\left(e^{A(t-s)} x\right)-\right. & \left.F^{\prime}\left(e^{A(t-s)} x\right)\right] e^{A(t-s)} F_{I}(x) \|_{H_{-\vartheta}} \leq \frac{\left.g_{2}(x)\|F\|_{C_{b}^{2}(H, H-\vartheta}^{2}\left\|P_{\mathbb{H} \backslash I}\right\|_{L(H, H-\rho}\right)}{(t-s)^{\rho+\vartheta}} \\
\text { and } \quad & {\left[\sum_{b \in \mathbb{U}}\left\|\left[F_{I}^{\prime}\left(e^{A(t-s)} x\right)-F^{\prime}\left(e^{A(t-s)} x\right)\right] e^{A(t-s)} B_{I}^{b}(x)\right\|_{H_{-\vartheta}}^{2}\right]^{\frac{1}{2}} } \\
& \leq \frac{g_{2}(x)\|F\|_{C_{b}^{2}\left(H, H_{-\vartheta}\right)}\|B\|_{C_{b}^{1}\left(H, H S\left(U, H_{-\vartheta}\right)\right.}\left\|P_{\mathbb{H} \backslash I}\right\|_{L\left(H, H_{-\rho}\right)}}{(t-s)^{\left(\rho+\frac{\vartheta}{2}\right)}} .
\end{aligned}
$$

Moreover, note that for all $x \in H, s, t \in[0, T]$ with $s<t$ it holds that

$$
\begin{aligned}
\sum_{b \in \mathbb{U}} & \left\|\left[F_{I}^{\prime \prime}\left(e^{A(t-s)} x\right)-F^{\prime \prime}\left(e^{A(t-s)} x\right)\right]\left(e^{A(t-s)} B_{I}^{b}(x), e^{A(t-s)} B_{I}^{b}(x)\right)\right\|_{H_{-\vartheta}} \\
\leq & \sum_{b \in \mathbb{U}}\left\|F^{\prime \prime}\left(e^{A(t-s)} P_{I}(x)\right)\left(\left[\operatorname{Id}_{H}+P_{I}\right] e^{A(t-s)} B_{I}^{b}(x),\left[\operatorname{Id}_{H}-P_{I}\right] e^{A(t-s)} B_{I}^{b}(x)\right)\right\|_{H_{-\vartheta}} \\
& +\sum_{b \in \mathbb{U}}\left\|\left[F^{\prime \prime}\left(e^{A(t-s)} P_{I}(x)\right)-F^{\prime \prime}\left(e^{A(t-s)} x\right)\right]\left(e^{A(t-s)} B_{I}^{b}(x), e^{A(t-s)} B_{I}^{b}(x)\right)\right\|_{H_{-\vartheta}} \\
\leq & 2\|F\|_{C_{b}^{2}\left(H, H_{-\vartheta}\right)}\left\|e^{A(t-s)} B_{I}(x)\right\|_{H S(U, H)}\left\|P_{\mathbb{H} \backslash I} e^{A(t-s)} B_{I}(x)\right\|_{H S(U, H)} \\
& +\frac{|F|_{C_{b}^{3}\left(H, H_{-\vartheta}\right)} g_{1}(x)\left\|P_{\mathbb{H} \backslash I}\right\|_{L\left(H, H_{-\rho}\right)}\left\|e^{A(t-s)} B_{I}(x)\right\|_{H S(U, H)}^{2}}{(t-s)^{\rho}} \\
\leq & \frac{2\|F\|_{C_{b}^{2}\left(H, H_{-\vartheta}\right)}\|B\|_{C_{b}^{1}\left(H, H S\left(U, H_{-\vartheta / 2}\right)\right)}^{2}\left\|P_{\mathbb{H} \backslash I}\right\|_{L\left(H, H_{-\rho}\right)} g_{2}(x)}{(t-s)^{\rho+\vartheta}} \\
& +\frac{|F|_{C_{b}^{3}\left(H, H_{-\vartheta}\right)}\|B\|_{C_{b}^{1}\left(H, H S\left(U, H_{-\vartheta / 2}\right)\right)}^{2}\left\|P_{\mathbb{H} \backslash I}\right\|_{L\left(H, H_{-\rho}\right)} g_{3}(x)}{(t-s)^{\rho+\vartheta}} \\
\leq & \frac{2\|F\|_{C_{b}^{3}\left(H, H_{-\vartheta}\right)}\|B\|_{C_{b}^{1}\left(H, H S\left(U, H_{-\vartheta / 2}\right)\right)}^{2}\left\|P_{\mathbb{H} \backslash I}\right\|_{L\left(H, H_{-\rho}\right)} g_{3}(x)}{(t-s)^{\rho+\vartheta}} .
\end{aligned}
$$

Putting (199), (200), and (201) into (197) proves that for all $x \in H, s, t \in[0, T)$ with 
$t>s$ it holds that

$$
\begin{aligned}
\left|\tilde{F}_{t, s}(x)\right| & \leq\left[c_{-\vartheta, 0}\|F\|_{C_{b}^{1}\left(H, H_{-\vartheta}\right)}^{2} g_{2}(x)+c_{-\vartheta}\|F\|_{C_{b}^{2}\left(H, H_{-\vartheta}\right)}^{2} g_{2}(x)\right. \\
& +c_{-\vartheta, 0,0}\|F\|_{C_{b}^{1}\left(H, H_{-\vartheta}\right)}\|B\|_{C_{b}^{1}\left(H, H S\left(U, H_{-\vartheta / 2}\right)\right.}^{2} g_{3}(x) \\
& +c_{-\vartheta, 0}\|B\|_{C_{b}^{1}\left(H, H S\left(U_{,} H_{-\vartheta / 2}\right)\right)}^{2} g_{3}(x)\|F\|_{C_{b}^{2}\left(H, H_{-\vartheta}\right)} \\
& \left.+c_{-\vartheta}\|B\|_{C_{b}^{1}\left(H, H S\left(U, H_{-\vartheta / 2}\right)\right)}^{2} g_{3}(x)\|F\|_{C_{b}^{3}\left(H, H_{-\vartheta}\right)}\right] \frac{\left\|P_{\mathbb{H} \backslash I}\right\|_{L_{\left(H, H_{-\rho}\right)}}}{(T-t)^{\vartheta}(t-s)^{(\rho+\vartheta)}}
\end{aligned}
$$

This implies that for all $t \in(0, T), s \in[0, t), x \in H$ it holds that

$$
\left|\tilde{F}_{t, s}(x)\right| \leq \frac{2\left[c_{-\vartheta}+c_{-\vartheta, 0}+c_{-\vartheta, 0,0}\right] \varsigma_{F, B} g_{3}(x)\left\|P_{\mathbb{H} \backslash I}\right\|_{L\left(H, H_{-\rho}\right)}}{(T-t)^{\vartheta}(t-s)^{\rho+\vartheta}} .
$$

This, in turn, proves that

$$
\begin{aligned}
& \left|\int_{0}^{T} \int_{0}^{t} \mathbb{E}\left[\tilde{F}_{t, s}\left(Y_{s}^{I}\right)\right] d s d t\right| \\
& \leq \frac{2 \varsigma_{F, B}\left\|P_{\mathbb{H} \backslash I}\right\|_{L\left(H, H_{-\rho}\right)} K_{3}^{I}\left[c_{-\vartheta}+c_{-\vartheta, 0}+c_{-\vartheta, 0,0}\right]}{(1-\rho-\vartheta)} \int_{0}^{T} \frac{t^{(1-\rho-\vartheta)}}{(T-t)^{\vartheta}} d t \\
& \leq \frac{2 T^{(1-\rho-\vartheta)} \varsigma_{F, B}\left\|P_{\mathbb{H} \backslash I}\right\|_{L\left(H, H_{-\rho}\right)} K_{3}^{I}\left[c_{-\vartheta}+c_{-\vartheta, 0}+c_{-\vartheta, 0,0}\right]}{(1-\rho-\vartheta)} \int_{0}^{T} \frac{1}{(T-t)^{\vartheta}} d t \\
& =\frac{2 T^{(2-\rho-2 \vartheta)} \varsigma_{F, B}\left\|P_{\mathbb{H} \backslash I}\right\|_{L\left(H, H_{-\rho}\right)} K_{3}^{I}\left[c_{-\vartheta}+c_{-\vartheta, 0}+c_{-\vartheta, 0,0}\right]}{(1-\rho-\vartheta)(1-\vartheta)} .
\end{aligned}
$$

It thus remains to bound the term $\frac{1}{2} \int_{0}^{T} \int_{0}^{t} \mathbb{E}\left[\tilde{B}_{t, s}\left(Y_{s}^{I}\right)\right] d s d t$ on the right hand side of (190). For this we estimate the terms which appear on the right hand side of (181) (cf. (216) and (217) below). We start by presenting a few auxiliary estimates for these terms. Note that for all $t \in(0, T], s \in[0, t), x, v \in H$ it holds that

$$
\begin{aligned}
& {\left[\sum_{b \in \mathbb{U}}\left\|\left[\left(B_{I}^{b}\right)^{\prime}\left(e^{A(t-s)} x\right)-\left(B^{b}\right)^{\prime}\left(e^{A(t-s)} x\right)\right] e^{A(t-s)} v\right\|_{H_{-\vartheta / 2}}^{2}\right]^{1 / 2}} \\
& =\left\|\left[B^{\prime}\left(e^{A(t-s)} P_{I}(x)\right) P_{I}-B^{\prime}\left(e^{A(t-s)} x\right)\right] e^{A(t-s)} v\right\|_{H S\left(U, H_{-\vartheta / 2}\right)} \\
& \leq\left\|\left[B^{\prime}\left(e^{A(t-s)} P_{I}(x)\right)-B^{\prime}\left(e^{A(t-s)} x\right)\right] P_{I} e^{A(t-s)} v\right\|_{H S\left(U, H_{-\vartheta / 2}\right)} \\
& \quad+\left\|B^{\prime}\left(e^{A(t-s)} x\right) P_{\mathbb{H} \backslash I} e^{A(t-s)} v\right\|_{H S\left(U, H_{-\vartheta / 2}\right)} .
\end{aligned}
$$

This shows that for all $r \in[0, \vartheta], t \in(0, T], s \in[0, t), x, v \in H$ it holds that

$$
\begin{aligned}
& {\left[\sum_{b \in \mathbb{U}}\left\|\left[\left(B_{I}^{b}\right)^{\prime}\left(e^{A(t-s)} x\right)-\left(B^{b}\right)^{\prime}\left(e^{A(t-s)} x\right)\right] e^{A(t-s)} v\right\|_{H_{-} / 2}^{2}\right]^{1 / 2}}
\end{aligned}
$$

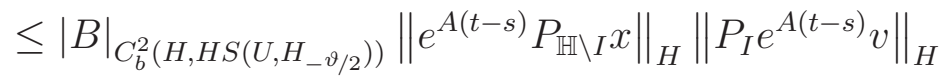

$$
\begin{aligned}
& +\|B\|_{C_{b}^{1}\left(H, H S\left(U, H_{-\vartheta / 2}\right)\right)}\left\|P_{\mathbb{H} \backslash I} e^{A(t-s)} v\right\|_{H} \\
& \leq\left[|B|_{C_{b}^{2}\left(H, H S\left(U_{\left., H_{-} \vartheta_{2}\right)}\right)\right.} g_{1}(x)+\|B\|_{C_{b}^{1}\left(H, H S\left(U_{, H_{-}, 2}\right)\right)}\right] \frac{\|v\|_{H_{-r}}\left\|P_{\mathbb{H} \backslash I}\right\|_{L\left(H, H_{-\rho}\right)}}{(t-s)^{(\rho+r)}}
\end{aligned}
$$

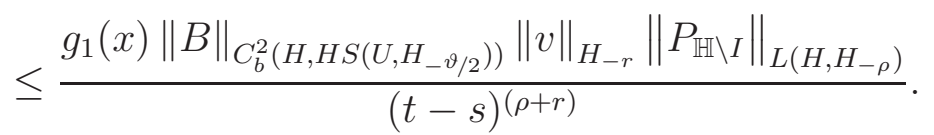


Hence, we obtain that for all $t \in(0, T], s \in[0, t), x, v \in H$ it holds that

$$
\begin{aligned}
& \sum_{b \in \mathbb{U}}\left\|\left[\left(B_{I}^{b}\right)^{\prime}\left(e^{A(t-s)} x\right)-\left(B^{b}\right)^{\prime}\left(e^{A(t-s)} x\right)\right] e^{A(t-s)} v\right\|_{H_{-\vartheta / 2}}^{2} \\
& \leq \frac{\left.\left|g_{1}(x)\right|^{2}\|B\|_{C_{b}^{2}\left(H, H S\left(U, H_{-} / 2\right)\right.}^{2}\right)\|v\|_{H_{-} / 2}^{2}\left\|P_{\mathbb{H} \backslash I}\right\|_{L\left(H, H_{-\rho}\right)}^{2}}{(t-s)^{(2 \rho+\vartheta)}} .
\end{aligned}
$$

We now estimate the first argument of the bilinear operator appearing in the first summand on the right hand side of (181). Observe that for all $r \in[0, \vartheta], t \in(0, T], s \in[0, t)$, $x, v \in H$ it holds that

$$
\begin{aligned}
& {\left[\sum_{b \in \mathbb{U}}\left\|\left[\left(B_{I}^{b}\right)^{\prime}\left(e^{A(t-s)} x\right)+\left(B^{b}\right)^{\prime}\left(e^{A(t-s)} x\right)\right] e^{A(t-s)} v\right\|_{H_{-} \vartheta / 2}^{2}\right]^{1 / 2}} \\
& =\left\|\left[B^{\prime}\left(e^{A(t-s)} P_{I}(x)\right) P_{I}+B^{\prime}\left(e^{A(t-s)} x\right)\right] e^{A(t-s)} v\right\|_{H S\left(U, H_{-\vartheta / 2}\right)}\|v\|_{H_{-r}} \\
& \leq 2\|B\|_{C_{b}^{1}\left(H, H S\left(U, H_{-\vartheta / 2}\right)\right)}\left\|e^{A(t-s)} v\right\|_{H} \leq \frac{\left.2\|B\|_{C_{b}^{1}\left(H, H S\left(U, H_{-} / 2\right)\right.}\right)}{(t-s)^{r}}
\end{aligned}
$$

This implies that for all $t \in(0, T], s \in[0, t), x \in H$ it holds that

$$
\begin{aligned}
& {\left[\sum_{b \in \mathbb{U}}\left\|\left[\left(B_{I}^{b}\right)^{\prime}\left(e^{A(t-s)} x\right)+\left(B^{b}\right)^{\prime}\left(e^{A(t-s)} x\right)\right] e^{A(t-s)} F_{I}(x)\right\|_{H_{-\vartheta / 2}}^{2}\right]^{1 / 2}} \\
& \leq \frac{\left.2\|B\|_{C_{b}^{1}\left(H, H S\left(U, H_{-\vartheta / 2}\right)\right.}\right)}{(t-s)^{\vartheta}} \leq F_{I}(x) \|_{H_{-\vartheta}} \leq \frac{2 g_{1}(x)\|B\|_{C_{b}^{1}\left(H, H S\left(U, H_{-\vartheta / 2}\right)\right)}\|F\|_{C_{b}^{1}\left(H, H_{-\vartheta}\right)}}{(t-s)^{\vartheta}}
\end{aligned}
$$

Next we use (208) to estimate the first argument of the bilinear operator appearing in the fourth summand on the right hand side of (181) and the first argument of the bilinear operator appearing in the seventh summand on the right hand side of (181). More specifically, note that (208) ensures that for all $t \in(0, T], s \in[0, t), x, v \in H$ it holds that

$$
\begin{aligned}
& \sum_{b \in \mathbb{U}}\left\|\left[\left(B_{I}^{b}\right)^{\prime}\left(e^{A(t-s)} x\right)+\left(B^{b}\right)^{\prime}\left(e^{A(t-s)} x\right)\right] e^{A(t-s)} v\right\|_{H_{-\vartheta / 2}}^{2} \\
& \leq \frac{4\|B\|_{C_{b}^{1}\left(H, H S\left(U, H_{-\vartheta / 2}\right)\right)}^{2}\|v\|_{H_{-\vartheta / 2}}^{2}}{(t-s)^{\vartheta}} .
\end{aligned}
$$

This shows that for all $t \in(0, T], s \in[0, t), x \in H$ it holds that

$$
\begin{aligned}
& {\left[\sum_{b_{1}, b_{2} \in \mathbb{U}}\left\|\left[\left(B_{I}^{b_{2}}\right)^{\prime}\left(e^{A(t-s)} x\right)+\left(B^{b_{2}}\right)^{\prime}\left(e^{A(t-s)} x\right)\right] e^{A(t-s)} B_{I}^{b_{1}}(x)\right\|_{H_{-\vartheta / 2}}^{2}\right]^{1 / 2}} \\
& =\left[\sum_{b_{1} \in \mathbb{U}}\left(\sum_{b_{2} \in \mathbb{U}}\left\|\left[\left(B_{I}^{b_{2}}\right)^{\prime}\left(e^{A(t-s)} x\right)+\left(B^{b_{2}}\right)^{\prime}\left(e^{A(t-s)} x\right)\right] e^{A(t-s)} B_{I}^{b_{1}}(x)\right\|_{H_{-\vartheta / 2}}^{2}\right)\right]^{1 / 2} \\
& \leq\left[\sum_{b_{1} \in \mathbb{U}}\left(\frac{4\|B\|_{C_{b}^{1}\left(H, H S\left(U, H_{-\vartheta / 2}\right)\right.}^{2}\left\|B_{I}^{b_{1}}(x)\right\|_{H_{-\vartheta / 2}}^{2}}{(t-s)^{\vartheta}}\right)\right]^{1 / 2} \\
& =\frac{2\|B\|_{C_{b}^{1}\left(H, H S\left(U, H_{-\vartheta / 2}\right)\right)}\left\|B_{I}(x)\right\|_{H S\left(U, H_{-\vartheta / 2}\right)}}{(t-s)^{\frac{\vartheta}{2}}} \leq \frac{2 g_{1}(x)\|B\|_{C_{b}^{1}\left(H, H S\left(U, H_{-\vartheta / 2}\right)\right)}^{2}}{(t-s)^{\frac{\vartheta}{2}}}
\end{aligned}
$$

To estimate the second argument of the bilinear operator appearing in the second summand on the right hand side of (181) we employ (206) to obtain that for all $t \in(0, T]$, 
$s \in[0, t), x \in H$ it holds that

$$
\begin{aligned}
& {\left[\sum_{b \in \mathbb{U}}\left\|\left[\left(B_{I}^{b}\right)^{\prime}\left(e^{A(t-s)} x\right)-\left(B^{b}\right)^{\prime}\left(e^{A(t-s)} x\right)\right] e^{A(t-s)} F_{I}(x)\right\|_{H_{-\vartheta / 2}}^{2}\right]^{1 / 2}} \\
& \leq \frac{g_{1}(x)\|B\|_{C_{b}^{2}\left(H, H S\left(U, H_{-\vartheta / 2}\right)\right.}\left\|F_{I}(x)\right\|_{H_{-\vartheta}}\left\|P_{\mathbb{H} \backslash I}\right\|_{L\left(H, H_{-\rho}\right)}}{(t-s)^{(\rho+\vartheta)}} \\
& \leq \frac{g_{2}(x)\|B\|_{C_{b}^{2}\left(H, H S\left(U, H_{-\vartheta / 2}\right)\right.}\|F\|_{C_{b}^{1}\left(H_{, H} H_{-\vartheta}\right)}\left\|P_{\mathbb{H} \backslash I}\right\|_{L\left(H, H_{-\rho}\right)}}{(t-s)^{(\rho+\vartheta)}} .
\end{aligned}
$$

In addition, we use (207) to estimate the second argument of the bilinear operator appearing in the fourth summand on the right hand side of (181) and the second argument of the trilinear operator appearing in the eighth summand on the right hand side of (181). More specifically, observe that (207) shows that for all $t \in(0, T], s \in[0, t), x \in H$ it holds that

$$
\begin{aligned}
& {\left[\sum_{b_{1}, b_{2} \in \mathbb{U}}\left\|\left[\left(B_{I}^{b_{2}}\right)^{\prime}\left(e^{A(t-s)} x\right)-\left(B^{b_{2}}\right)^{\prime}\left(e^{A(t-s)} x\right)\right] e^{A(t-s)} B_{I}^{b_{1}}(x)\right\|_{H_{-\vartheta / 2}}^{2}\right]^{1 / 2}} \\
& =\left[\sum_{b_{1} \in \mathbb{U}}\left(\sum_{b_{2} \in \mathbb{U}}\left\|\left[\left(B_{I}^{b_{2}}\right)^{\prime}\left(e^{A(t-s)} x\right)-\left(B^{b_{2}}\right)^{\prime}\left(e^{A(t-s)} x\right)\right] e^{A(t-s)} B_{I}^{b_{1}}(x)\right\|_{H_{-\vartheta} / 2}^{2}\right)\right]^{1 / 2} \\
& \leq\left[\sum_{b_{1} \in \mathbb{U}}\left(\frac{\left|g_{1}(x)\right|^{2}\|B\|_{C_{b}^{2}\left(H, H S\left(U, H_{-\vartheta / 2}\right)\right)}^{2}\left\|B_{I}^{b_{1}}(x)\right\|_{H_{-\vartheta / 2}}^{2}\left\|P_{\mathbb{H} \backslash I}\right\|_{L\left(H, H_{-}\right)}^{2}}{(t-s)^{(2 \rho+\vartheta)}}\right)\right]^{1 / 2} \\
& =\frac{g_{1}(x)\|B\|_{C_{b}^{2}\left(H, H S\left(U, H_{-\vartheta / 2}\right)\right)}\left\|B_{I}(x)\right\|_{H S\left(U, H_{-\vartheta / 2}\right)}\left\|P_{\mathbb{H} \backslash I}\right\|_{L\left(H, H_{-}\right)}}{(t-s)^{\left(\rho+\frac{\vartheta}{2}\right)}} \\
& \leq \frac{g_{2}(x)\|B\|_{C_{b}^{2}\left(H, H S\left(U, H_{-\vartheta / 2}\right)\right.}\|B\|_{C_{b}^{1}\left(H, H S\left(U, H_{-\vartheta / 2}\right)\right)}\left\|P_{\mathbb{H} \backslash I}\right\|_{L\left(H, H_{-\rho}\right)}}{(t-s)^{\left(\rho+\frac{\vartheta}{2}\right)}} .
\end{aligned}
$$

We next estimate the second argument of the bilinear operator appearing in the fifth summand on the right hand side of (181) and the second argument of the bilinear operator appearing in the sixth summand on the right hand side of (181). Observe that for all $x \in H, t \in(0, T], s \in[0, t)$ it holds that

$$
\begin{aligned}
& \sum_{b_{1} \in \mathbb{U}}\left[\sum_{b_{2} \in \mathbb{U}}\left\|\left[\left(B_{I}^{b_{2}}\right)^{\prime \prime}\left(e^{A(t-s)} x\right)+\left(B^{b_{2}}\right)^{\prime \prime}\left(e^{A(t-s)} x\right)\right]\left(e^{A(t-s)} B_{I}^{b_{1}}(x), e^{A(t-s)} B_{I}^{b_{1}}(x)\right)\right\|_{H_{-\vartheta / 2}}^{2}\right]^{1 / 2} \\
& \left.=\sum_{b_{1} \in \mathbb{U}}\left\|\left[\left(B_{I}\right)^{\prime \prime}\left(e^{A(t-s)} x\right)+B^{\prime \prime}\left(e^{A(t-s)} x\right)\right]\left(e^{A(t-s)} B_{I}^{b_{1}}(x), e^{A(t-s)} B_{I}^{b_{1}}(x)\right)\right\|_{H S\left(U, H_{-} \vartheta_{2}\right.}\right) \\
& \left.\leq \sum_{b_{1} \in \mathbb{U}}\left\|B^{\prime \prime}\left(e^{A(t-s)} P_{I}(x)\right)\left(P_{I} e^{A(t-s)} B_{I}^{b_{1}}(x), P_{I} e^{A(t-s)} B_{I}^{b_{1}}(x)\right)\right\|_{H S\left(U, H_{-} / 2\right.}\right) \\
& +\sum_{b_{1} \in \mathbb{U}}\left\|B^{\prime \prime}\left(e^{A(t-s)} x\right)\left(e^{A(t-s)} B_{I}^{b_{1}}(x), e^{A(t-s)} B_{I}^{b_{1}}(x)\right)\right\|_{H S\left(U, H_{-\vartheta / 2}\right)}
\end{aligned}
$$

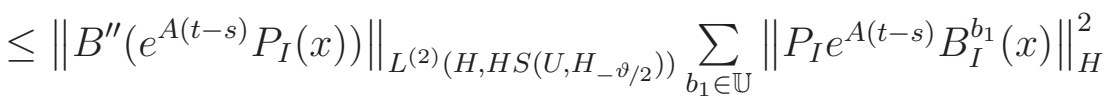

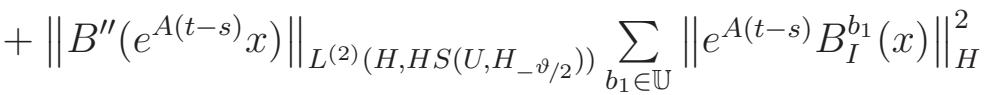

$$
\begin{aligned}
& \leq\|B\|_{C_{b}^{2}\left(H, H S\left(U, H_{-\vartheta / 2}\right)\right)}\left[\left\|P_{I} e^{A(t-s)} B_{I}(x)\right\|_{H S(U, H)}^{2}+\left\|e^{A(t-s)} B_{I}(x)\right\|_{H S(U, H)}^{2}\right] \\
& \leq \frac{2\|B\|_{C_{b}^{2}\left(H, H S\left(U, H_{-\vartheta / 2}\right)\right)}\|B\|_{C_{b}^{1}\left(H, H S\left(U, H_{-\vartheta / 2}\right)\right)}^{2} g_{2}(x)}{(t-s)^{\vartheta}} \text {, }
\end{aligned}
$$


and

$$
\begin{aligned}
& \sum_{b_{1} \in \mathbb{U}}\left[\sum_{b_{2} \in \mathbb{U}}\left\|\left[\left(B_{I}^{b_{2}}\right)^{\prime \prime}\left(e^{A(t-s)} x\right)-\left(B^{b_{2}}\right)^{\prime \prime}\left(e^{A(t-s)} x\right)\right]\left(e^{A(t-s)} B_{I}^{b_{1}}(x), e^{A(t-s)} B_{I}^{b_{1}}(x)\right)\right\|_{H_{-\vartheta / 2}}^{2}\right]^{1 / 2} \\
& \left.=\sum_{b_{1} \in \mathbb{U}}\left\|\left[\left(B_{I}\right)^{\prime \prime}\left(e^{A(t-s)} x\right)-B^{\prime \prime}\left(e^{A(t-s)} x\right)\right]\left(e^{A(t-s)} B_{I}^{b_{1}}(x), e^{A(t-s)} B_{I}^{b_{1}}(x)\right)\right\|_{H S\left(U, H_{-} \vartheta / 2\right.}\right) \\
& \left.\leq \sum_{b_{1} \in \mathbb{U}}\left\|\left[B^{\prime \prime}\left(e^{A(t-s)} P_{I}(x)\right)-B^{\prime \prime}\left(e^{A(t-s)} x\right)\right]\left(e^{A(t-s)} B_{I}^{b_{1}}(x), e^{A(t-s)} B_{I}^{b_{1}}(x)\right)\right\|_{H S\left(U, H_{-} / 2\right.}\right) \\
& +\sum_{b_{1} \in \mathbb{U}}\left\|B^{\prime \prime}\left(e^{A(t-s)} P_{I}(x)\right)\left(\left(\operatorname{Id}_{H}-P_{I}\right) e^{A(t-s)} B_{I}^{b_{1}}(x),\left(\operatorname{Id}_{H}+P_{I}\right) e^{A(t-s)} B_{I}^{b_{1}}(x)\right)\right\|_{H S\left(U, H_{-\vartheta / 2}\right)} \\
& \leq\left\|B^{\prime \prime}\left(e^{A(t-s)} P_{I}(x)\right)-B^{\prime \prime}\left(e^{A(t-s)} x\right)\right\|_{L^{(2)}\left(H, H S\left(U, H_{-\vartheta / 2}\right)\right)} \sum_{b_{1} \in \mathbb{U}}\left\|e^{A(t-s)} B_{I}^{b_{1}}(x)\right\|_{H}^{2}
\end{aligned}
$$

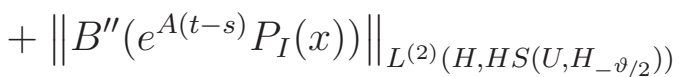

$$
\begin{aligned}
& \cdot \sum_{b_{1} \in \mathbb{U}}\left[\left\|\left(\operatorname{Id}_{H}-P_{I}\right) e^{A(t-s)} B_{I}^{b_{1}}(x)\right\|_{H}\left\|\left(\operatorname{Id}_{H}+P_{I}\right) e^{A(t-s)} B_{I}^{b_{1}}(x)\right\|_{H}\right]
\end{aligned}
$$

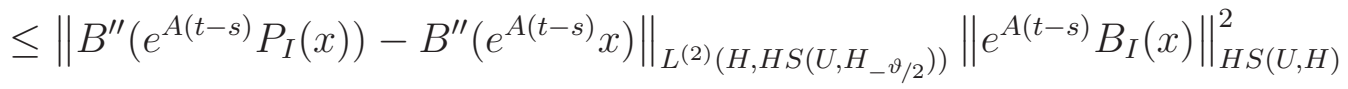

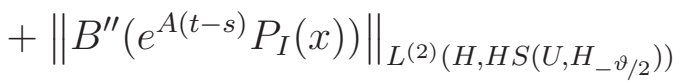

$$
\begin{aligned}
& \text { - }\left\|\left(\operatorname{Id}_{H}-P_{I}\right) e^{A(t-s)} B_{I}(x)\right\|_{H S(U, H)}\left\|\left(\operatorname{Id}_{H}+P_{I}\right) e^{A(t-s)} B_{I}(x)\right\|_{H S(U, H)} \\
& \leq \frac{|B|_{C_{b}^{3}\left(H, H S\left(U, H_{-\vartheta / 2}\right)\right.}\|B\|_{C_{b}^{1}\left(H, H S\left(U, H_{-\vartheta / 2}\right)\right.}^{2}\left\|P_{\mathbb{H} \backslash I}\right\|_{L\left(H, H_{-\rho}\right)} g_{3}(x)}{(t-s)^{(\rho+\vartheta)}}
\end{aligned}
$$

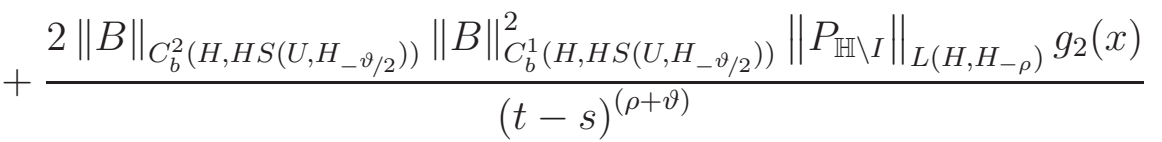

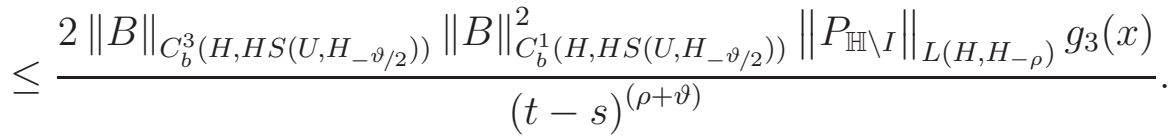

Putting (209) and (211)-(215) into (181) shows that for all $t \in(0, T), s \in[0, t), x \in H$ it holds that

$$
\begin{aligned}
& \left|\tilde{B}_{t, s}(x)\right| \\
& \leq\left[2 c_{-\vartheta / 2,-\vartheta / 2}\|F\|_{C_{b}^{1}\left(H, H_{-\vartheta}\right)}|B|_{C_{b}^{1}\left(H, H S\left(U, H_{-\vartheta / 2}\right)\right)}\|B\|_{C_{b}^{1}\left(H, H S\left(U, H_{-\vartheta / 2}\right)\right)} g_{2}(x)\right.
\end{aligned}
$$

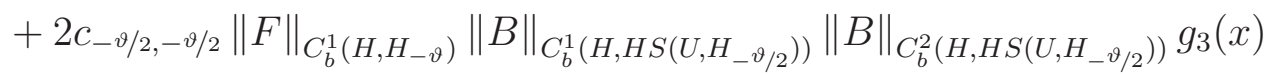

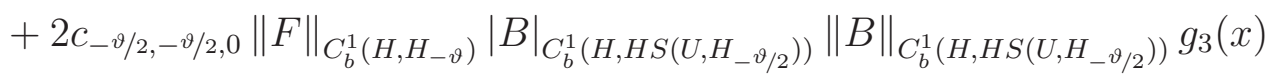

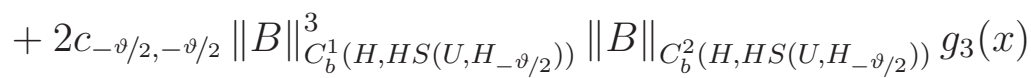

$$
\begin{aligned}
& +c_{-\vartheta / 2,-\vartheta / 2}|B|_{C_{b}^{1}\left(H, H S\left(U, H_{-\vartheta / 2}\right)\right)}\|B\|_{C_{b}^{1}\left(H, H S\left(U, H_{-\vartheta / 2}\right)\right)}^{2}\|B\|_{C_{b}^{2}\left(H, H S\left(U, H_{-} \vartheta_{2}\right)\right)} g_{3}(x) \\
& \left.+2 c_{-\vartheta / 2,-\vartheta / 2}\|B\|_{C_{b}^{1}\left(H, H S\left(U, H_{-} \vartheta / 2\right)\right.}^{3}\|B\|_{C_{b}^{3}\left(H, H S\left(U_{,} H_{-} / 2\right)\right.}\right) g_{4}(x) \\
& +2 c_{-\vartheta / 2,-\vartheta / 2,0}|B|_{C_{b}^{1}\left(H, H S\left(U, H_{-} \vartheta / 2\right)\right.}\|B\|_{C_{b}^{1}\left(H, H S\left(U_{,} H_{-} / 2\right)\right.}^{3} g_{3}(x)
\end{aligned}
$$

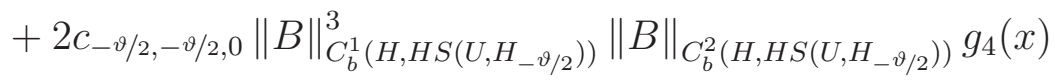

$$
\begin{aligned}
& \left.+c_{-\vartheta / 2,-\vartheta / 2,0,0}|B|_{C_{b}^{1}\left(H, H S\left(U, H_{-\vartheta / 2}\right)\right.}\|B\|_{C_{b}^{1}\left(H, H S\left(U, H_{-\vartheta / 2}\right)\right)}^{3} g_{4}(x)\right] \frac{\left\|P_{\mathbb{H} \backslash I}\right\|_{L\left(H, H_{-\rho}\right)}}{(T-t)^{\vartheta}(t-s)^{(\rho+\vartheta)}} .
\end{aligned}
$$


This implies that for all $t \in(0, T), s \in[0, t), x \in H$ it holds that

$$
\left|\tilde{B}_{t, s}(x)\right| \leq \frac{9\left[c_{-\vartheta / 2,-\vartheta / 2}+c_{-\vartheta / 2,-\vartheta / 2,0}+c_{-\vartheta / 2,-\vartheta / 2,0,0}\right] \varsigma_{F, B} g_{4}(x)\left\|P_{\mathbb{H} \backslash I}\right\|_{L\left(H, H_{-\rho}\right)}}{(T-t)^{\vartheta}(t-s)^{\rho+\vartheta}} .
$$

This proves that

$$
\begin{aligned}
& \frac{1}{2}\left|\int_{0}^{T} \int_{0}^{t} \mathbb{E}\left[\tilde{B}_{t, s}\left(Y_{s}^{I}\right)\right] d s d t\right| \\
& \leq \frac{9 T^{(2-\rho-2 \vartheta)} \varsigma_{F, B}\left\|P_{\mathbb{H} \backslash I}\right\|_{L\left(H_{\left., H_{-\rho}\right)}\right.}}{2(1-\rho-\vartheta)(1-\vartheta)}\left[c_{-\vartheta / 2,-\vartheta / 2}+c_{-\vartheta / 2,-\vartheta / 2,0}+c_{-\vartheta / 2,-\vartheta / 2,0,0}\right] K_{4}^{I} .
\end{aligned}
$$

Putting (193), (196), (204), and (218) into (190) finally yields

$$
\begin{aligned}
& \left|\mathbb{E}\left[\varphi\left(X_{T}^{\mathbb{H}}\right)\right]-\mathbb{E}\left[\varphi\left(Y_{T}^{I}\right)\right]\right| \\
& \leq\left[c_{-\vartheta}+c_{-\vartheta, 0}+c_{-\vartheta, 0,0}+c_{-\vartheta / 2,-\vartheta / 2}+c_{-\vartheta / 2,-\vartheta / 2,0}+c_{-\vartheta / 2,-\vartheta / 2,0,0}\right] \\
& \quad \cdot \frac{T^{(1-\vartheta-\rho)}}{(1-\vartheta-\rho)}\left[1+\frac{9 T^{(1-\vartheta)}}{2(1-\vartheta)}\right] \varsigma_{F, B} K_{4}^{I}\left\|P_{\mathbb{H} \backslash I}\right\|_{L\left(H, H_{-\rho}\right)} .
\end{aligned}
$$

This establishes the first inequality of (162). The second inequality of (162) follows from Lemma 3.4 and the assumption that $\vartheta<1 / 2$. The proof of Lemma 3.7 is thus completed.

The next result, Corollary 3.8 , is an immediate consequence of Lemma 3.5 and Lemma 3.7 above.

Corollary 3.8. Assume the setting in Section 3.2 and let $\rho \in[0,1-\vartheta), I \in \mathcal{P}(\mathbb{H})$. Then

$$
\begin{aligned}
& \left|\mathbb{E}\left[\varphi\left(X_{T}^{\mathbb{H}}\right)\right]-\mathbb{E}\left[\varphi\left(X_{T}^{I}\right)\right]\right| \leq \frac{9}{2 T^{\rho}}\left[1+\frac{T^{(1-\vartheta)}}{(1-\vartheta-\rho)}\right]^{2}\left\|P_{\mathbb{H} \backslash I}\right\|_{L\left(H, H_{-\rho}\right)} \varsigma_{F, B} K_{4}^{I} \\
& \cdot\left[\|\varphi\|_{C_{b}^{3}(H, \mathbb{R})}+c_{-\vartheta}+c_{-\vartheta, 0}+c_{-\vartheta, 0,0}+c_{-\vartheta / 2,-\vartheta / 2}+c_{-\vartheta / 2,-\vartheta / 2,0}+c_{-\vartheta / 2,-\vartheta / 2,0,0}\right]<\infty .
\end{aligned}
$$

In the proof of the next result, Corollary 3.9 below, we employ an upper bound result for the real numbers $K_{4}^{I}, I \in \mathcal{P}(\mathbb{H})$, to obtain a further upper bound for the real numbers $\left|\mathbb{E}\left[\varphi\left(X_{T}^{\mathbb{H}}\right)\right]-\mathbb{E}\left[\varphi\left(X_{T}^{I}\right)\right]\right|, I \in \mathcal{P}(\mathbb{H})$. For the formulation of Corollary 3.9 we recall that for all $x \in[0, \infty), \theta \in[0,1)$ it holds that $\mathcal{E}_{(1-\theta)}(x)=\left[\sum_{n=0}^{\infty} \frac{x^{2 n} \Gamma(1-\theta)^{n}}{\Gamma(n(1-\theta)+1)}\right]^{1 / 2}$ (see Section 1.3).

Corollary 3.9. Assume the setting in Section [3.2. Then it holds for every $\theta \in[0,1)$, $\rho \in[0,1-\vartheta), I \in \mathcal{P}(\mathbb{H})$ that

$$
\begin{aligned}
& \left|\mathbb{E}\left[\varphi\left(X_{T}^{\mathbb{H}}\right)\right]-\mathbb{E}\left[\varphi\left(X_{T}^{I}\right)\right]\right| \leq \frac{18}{T^{\rho}}\left[1+\frac{T^{(1-\vartheta)}}{(1-\vartheta-\rho)}\right]^{2} \mathbb{E}\left[\max \left\{1,\|\xi\|_{H}^{4}\right\}\right]\left\|P_{\mathbb{H} \backslash I}\right\|_{L\left(H, H_{-\rho}\right)} \\
& \cdot \varsigma_{F, B}\left|\mathcal{E}_{(1-\theta)}\left[\frac{T^{1-\theta} \sqrt{2}\|F\|_{C_{b}^{1}\left(H, H_{-\theta}\right)}}{\sqrt{1-\theta}}+\sqrt{12 T^{1-\theta}}\|B\|_{C_{b}^{1}\left(H, H S\left(U, H_{-\theta / 2}\right)\right)}\right]\right|^{4} \\
& \cdot\left[\|\varphi\|_{C_{b}^{3}(H, \mathbb{R})}+c_{-\vartheta}+c_{-\vartheta, 0}+c_{-\vartheta, 0,0}+c_{-\vartheta / 2,-\vartheta / 2}+c_{-\vartheta / 2,-\vartheta / 2,0}+c_{-\vartheta / 2,-\vartheta / 2,0,0}\right]<\infty .
\end{aligned}
$$

Proof. Note that, e.g., Proposition 3.4 in Cox et al. [11] (with $H=H, U=U, \mathbb{H}=\mathbb{H}$, $\lambda=\lambda, A=A, T=T, p=4, \gamma=0, \eta=\theta, F=\left(H \ni v \mapsto F_{I}(v) \in H_{-\theta}\right)$, $B=\left(H \ni v \mapsto\left(U \ni u \mapsto B_{I}(v) u \in H_{-\theta / 2}\right) \in H S\left(U, H_{-\theta / 2}\right)\right), W=W, X=([0, T] \times \Omega \ni$ $\left.(t, \omega) \mapsto Y_{t}^{I}(\omega) \in H\right)$ for $I \in \mathcal{P}(\mathbb{H}), \theta \in[0,1)$ in the notation of Proposition 3.4 in [1] $)$ ensures that for all $\theta \in[0,1), I \in \mathcal{P}(\mathbb{H})$ it holds that

$$
\begin{aligned}
\left|K_{4}^{I}\right|^{1 / 4}= & \sup _{t \in[0, T]}\left\|\max \left\{1,\left\|Y_{t}^{I}\right\|_{H}\right\}\right\|_{L^{4}(\mathbb{P} ; \mathbb{R})} \leq \sqrt{2}\left\|\max \left\{1,\|\xi\|_{H}\right\}\right\|_{L^{4}(\mathbb{P} ; \mathbb{R})} \\
& \cdot \mathcal{E}_{(1-\theta)}\left[\frac{T^{1-\theta} \sqrt{2}\|F\|_{C_{b}^{1}\left(H_{,} H_{-\theta}\right)}}{\sqrt{1-\theta}}+\sqrt{12 T^{1-\theta}}\|B\|_{C_{b}^{1}\left(H, H S\left(U, H_{-\theta / 2}\right)\right)}\right]<\infty .
\end{aligned}
$$

This and (220) establish (221). The proof of Corollary 3.9 is thus completed. 
The next result, Corollary 3.10 below, is an immediate consequence of Corollary 3.9 and the fact that $\forall I \in \mathcal{P}(\mathbb{H}) \backslash\{\mathbb{H}\}, \rho \in[0, \infty):\left\|P_{\mathbb{H} \backslash I}\right\|_{L\left(H, H_{-}\right)}=\left[\inf _{b \in \mathbb{H} \backslash I}\left|\lambda_{b}\right|\right]^{-\rho}$.

Corollary 3.10. Assume the setting in Section 3.2. Then for every $\varepsilon \in(0, \infty)$ there exists a real number $C \in \mathbb{R}$ such that for all $I \in \mathcal{P}(\mathbb{H}) \backslash\{\mathbb{H}\}$ it holds that

$$
\left|\mathbb{E}\left[\varphi\left(X_{T}^{\mathbb{H}}\right)\right]-\mathbb{E}\left[\varphi\left(X_{T}^{I}\right)\right]\right| \leq C \cdot\left[\inf _{b \in \mathbb{H} \backslash I}\left|\lambda_{b}\right|\right]^{\varepsilon-(1-\vartheta)} .
$$

\section{Strong convergence of mollified solutions for SEEs}

In this section an elementary strong convergence result, see Proposition 4.1 below, is established. More specifically, in the framework of Section 1.4 we establish in Proposition 4.1 below an explicit upper bound for the strong approximation error

$$
\mathbb{E}\left[\left\|X_{T}^{0}-X_{T}^{\kappa}\right\|_{H}^{p}\right]
$$

for $\kappa \in[0, \infty), p \in[2, \infty)$ where $X^{\kappa}:[0, T] \times \Omega \rightarrow H, \kappa \in[0, \infty)$, are appropriate mild solution processes of (225). We have for every $\kappa \in(0, \infty)$ that $X^{\kappa}:[0, T] \times \Omega \rightarrow H$ is a suitably mollified version of $X^{0}:[0, T] \times \Omega \rightarrow H$ with the mollification parameter $\kappa \in(0, \infty)$. We prove Proposition 4.1 below by applying the strong perturbation estimate in Andersson et al. [2, Proposition 2.7]. Proposition 2.7 in [2], in turn, is established by using a generalized Gronwall inequality (see Henry [27, Exercise 3 in Chapter 7]). Proposition 4.1 below, in particular, allows us to prove estimate (15) in the introduction. In Section 5 below we will use Proposition 4.1 in conjunction with Corollary 3.9 in Section 3 to establish weak convergence rates for Galerkin approximations of SEEs. In Corollary 4.2 we further simplify the explicit bound obtained in Proposition 4.1 below.

\subsection{Setting}

Assume the setting in Section 1.4 and let $p \in[2, \infty), \vartheta \in[0,1), F \in \operatorname{Lip}^{0}\left(H, H_{-\vartheta}\right)$, $B \in \operatorname{Lip}^{0}\left(H, H S\left(U, H_{-\vartheta / 2}\right)\right), \xi \in L^{p}\left(\left.\mathbb{P}\right|_{\mathcal{F}_{0}} ; H\right)$.

The above assumptions ensure that there exist up-to-modifications unique $\left(\mathcal{F}_{t}\right)_{t \in[0, T]^{-}}$ predictable stochastic processes $X^{\kappa}:[0, T] \times \Omega \rightarrow H, \kappa \in[0, \infty)$, which satisfy that for all $\kappa \in[0, \infty)$ it holds that $\sup _{t \in[0, T]} \mathbb{E}\left[\left\|X_{t}^{\kappa}\right\|_{H}^{p}\right]<\infty$ and which satisfy that for all $t \in[0, T]$, $\kappa \in[0, \infty)$ it holds $\mathbb{P}$-a.s. that

$$
X_{t}^{\kappa}=e^{A t} \xi+\int_{0}^{t} e^{A(\kappa+t-s)} F\left(X_{s}^{\kappa}\right) d s+\int_{0}^{t} e^{A(\kappa+t-s)} B\left(X_{s}^{\kappa}\right) d W_{s} .
$$

\subsection{A strong convergence result}

Proposition 4.1. Assume the setting in Section 4.1 and let $\kappa \in[0, \infty), \rho \in\left[0, \frac{1-\vartheta}{2}\right)$. Then

$$
\begin{aligned}
& \left\|X_{T}^{0}-X_{T}^{\kappa}\right\|_{L^{p}(\mathbb{P} ; H)} \leq\left\|\max \left\{1,\|\xi\|_{H}\right\}\right\|_{L^{p}(\mathbb{P} ; \mathbb{R})} \\
& \cdot 2 \kappa^{\rho}\left|\mathcal{E}_{(1-\vartheta)}\left[\frac{T^{(1-\vartheta)} \sqrt{2}\|F\|_{\operatorname{Lip}^{0}(H, H-\vartheta)}}{\sqrt{1-\vartheta}}+\sqrt{T^{(1-\vartheta)} p(p-1)}\|B\|_{\operatorname{Lip}^{0}\left(H, H S\left(U, H_{-\vartheta / 2}\right)\right)}\right]\right|^{2} \\
& \cdot\left[\frac{T^{(1-\rho-\vartheta)}}{(1-\rho-\vartheta)}\|F\|_{\operatorname{Lip}^{0}\left(H, H_{-\vartheta}\right)}+\frac{\sqrt{p(p-1) T^{(1-2 \rho-\vartheta)}}}{\sqrt{2-4 \rho-2 \vartheta}}\|B\|_{\operatorname{Lip}^{0}\left(H, H S\left(U, H_{-\vartheta / 2}\right)\right)}\right]
\end{aligned}
$$


Proof. First of all, observe that Proposition 2.7 in [2] (with $H=H, U=U, T=T$, $\eta=0, p=p, \alpha=\vartheta, \hat{\alpha}=0, \beta=\vartheta / 2, \hat{\beta}=0, L_{0}=|F|_{\operatorname{Lip}^{0}\left(H, H_{-\vartheta}\right)}, \hat{L}_{0}=\|F(0)\|_{H_{-\vartheta}}$, $L_{1}=|B|_{\operatorname{Lip}^{0}\left(H, H S\left(U, H_{-\vartheta / 2}\right)\right)}+\varepsilon, \hat{L}_{1}=\|B(0)\|_{H S\left(U_{,} H_{-\vartheta / 2}\right)}, W=W, A=A, \mathbf{F}=([0, T] \times$ $\left.\Omega \times H \ni(t, \omega, v) \mapsto F(v) \in H_{-\vartheta}\right), \mathbf{B}=([0, T] \times \Omega \times H \ni(t, \omega, v) \mapsto(U \ni u \mapsto B(v) u \in$ $\left.\left.H_{-\vartheta / 2}\right) \in H S\left(U, H_{-\vartheta / 2}\right)\right), \delta=0, Y^{1}=X^{0}, Y^{2}=X^{\kappa}, \lambda=0$ for $\varepsilon \in(0, \infty)$ in the notation of Proposition 2.7 in [2]) shows that for all $\varepsilon \in(0, \infty)$ it holds that

$$
\begin{aligned}
& \left\|X_{T}^{0}-X_{T}^{\kappa}\right\|_{L^{p(\mathbb{P} ; H)}} \\
& \left.\leq \sqrt{2} \cdot \mathcal{E}_{(1-\vartheta)}\left[\frac{T^{(1-\vartheta)} \sqrt{2}|F|_{\operatorname{Lip}^{0}(H, H-\vartheta)}}{\sqrt{1-\vartheta}}+\sqrt{T^{(1-\vartheta)} p(p-1)}\left(|B|_{\operatorname{Lip}^{0}\left(H, H S\left(U_{,} H_{-\vartheta}\right)\right.}\right)+\varepsilon\right)\right] \\
& \cdot \sup _{t \in[0, T]}\left\|\int_{0}^{t} e^{A(t-s)}\left(\operatorname{Id}_{H}-e^{A \kappa}\right) F\left(X_{s}^{\kappa}\right) d s+\int_{0}^{t} e^{A(t-s)}\left(\operatorname{Id}_{H}-e^{A \kappa}\right) B\left(X_{s}^{\kappa}\right) d W_{s}\right\|_{L^{p}(\mathbb{P} ; H)} .
\end{aligned}
$$

In the next step we observe that the Burkholder-Davis-Gundy type inequality in Lemma 7.7 in Da Prato \& Zabczyk [15], Lemma 1.2, and the fact that for all $r \in[0,1]$ it holds that $\sup _{t \in(0, \infty)}\left\|(-t A)^{-r}\left(\operatorname{Id}_{H}-e^{A t}\right)\right\|_{L(H)} \leq 1$ imply that for all $t \in[0, T], r \in[0,1-\vartheta)$ it holds that

$$
\begin{aligned}
& \left\|\int_{0}^{t} e^{A(t-s)}\left(\operatorname{Id}_{H}-e^{A \kappa}\right) F\left(X_{s}^{\kappa}\right) d s\right\|_{L^{p}(\mathbb{P} ; H)} \\
& \leq \frac{T^{(1-r-\vartheta)}}{(1-r-\vartheta)}\left[\sup _{s \in[0, T]}\left\|F\left(X_{s}^{\kappa}\right)\right\|_{L^{p}\left(\mathbb{P} ; H_{-\vartheta}\right)}\right] \kappa^{r} \\
& \leq \frac{T^{(1-r-\vartheta)}}{(1-r-\vartheta)}\|F\|_{\operatorname{Lip}^{0}\left(H, H_{-\vartheta}\right)} \max \left\{1, \sup _{s \in[0, T]}\left\|X_{s}^{\kappa}\right\|_{L^{p}(\mathbb{P} ; H)}\right\} \kappa^{r}
\end{aligned}
$$

and that for all $t \in[0, T], r \in\left[0, \frac{1-\vartheta}{2}\right)$ it holds that

$$
\begin{aligned}
& \left\|\int_{0}^{t} e^{A(t-s)}\left(\operatorname{Id}_{H}-e^{A \kappa}\right) B\left(X_{s}^{\kappa}\right) d W_{s}\right\|_{L^{p}(\mathbb{P} ; H)} \\
& \leq \sqrt{\frac{p(p-1)}{2}} \frac{\sqrt{T^{(1-2 r-\vartheta)}}}{\sqrt{1-2 r-\vartheta}}\left[\sup _{s \in[0, T]}\left\|B\left(X_{s}^{\kappa}\right)\right\|_{L^{p}\left(\mathbb{P} ; H S\left(U, H_{-\vartheta / 2}\right)\right)}\right] \kappa^{r} \\
& \leq \sqrt{\frac{p(p-1)}{2}} \frac{\sqrt{T^{(1-2 r-\vartheta)}}}{\sqrt{1-2 r-\vartheta}}\|B\|_{L^{0} p^{0}\left(H, H S\left(U, H_{-\vartheta / 2}\right)\right)} \max \left\{1, \sup _{s \in[0, T]}\left\|X_{s}^{\kappa}\right\|_{L^{p}(\mathbb{P} ; H)}\right\} \kappa^{r} .
\end{aligned}
$$


Putting (228) and (229) into (227) yields that for all $r \in\left[0, \frac{1-\vartheta}{2}\right)$ it holds that

$$
\begin{aligned}
& \left\|X_{T}^{0}-X_{T}^{\kappa}\right\|_{L^{p}(\mathbb{P} ; H)} \leq \sqrt{2} \kappa^{r} \max \left\{1, \sup _{t \in[0, T]}\left\|X_{t}^{\kappa}\right\|_{L^{p}(\mathbb{P} ; H)}\right\} \\
& \cdot\left(\inf _{\varepsilon \in(0, \infty)} \mathcal{E}_{(1-\vartheta)}\left[\frac{T^{(1-\vartheta)} \sqrt{2}|F|_{\operatorname{Lip}^{0}\left(H, H_{-\vartheta}\right)}}{\sqrt{1-\vartheta}}+\sqrt{T^{(1-\vartheta)} p(p-1)}|B|_{\operatorname{Lip}^{0}\left(H, H S\left(U, H_{-\vartheta}\right)\right.}+\varepsilon\right]\right) \\
& \cdot\left[\frac{T^{(1-r-\vartheta)}}{(1-r-\vartheta)}\|F\|_{\operatorname{Lip}^{0}\left(H, H_{-\vartheta}\right)}+\frac{\sqrt{p(p-1) T^{(1-2 r-\vartheta)}}}{\sqrt{2-4 r-2 \vartheta}}\|B\|_{\operatorname{Lip}^{0}\left(H, H S\left(U, H_{-\vartheta / 2}\right)\right)}\right] \\
& \leq \sqrt{2} \kappa^{r}\left[\sup _{t \in[0, T]}\left\|\max \left\{1,\left\|X_{t}^{\kappa}\right\|_{H}\right\}\right\|_{L^{p}(\mathbb{P} ; \mathbb{R})}\right] \\
& \cdot \mathcal{E}_{(1-\vartheta)}\left[\frac{T^{(1-\vartheta)} \sqrt{2}|F|_{\operatorname{Lip}^{0}\left(H, H_{-\vartheta}\right)}}{\sqrt{1-\vartheta}}+\sqrt{T^{(1-\vartheta)} p(p-1)}|B|_{\operatorname{Lip}^{0}\left(H, H S\left(U, H_{-\vartheta / 2}\right)\right)}\right] \\
& \cdot\left[\frac{T^{(1-r-\vartheta)}}{(1-r-\vartheta)}\|F\|_{\operatorname{Lip}^{0}\left(H, H_{-\vartheta}\right)}+\frac{\sqrt{p(p-1) T^{(1-2 r-\vartheta)}}}{\sqrt{2-4 r-2 \vartheta}}\|B\|_{\operatorname{Lip}^{0}\left(H, H S\left(U, H_{-\vartheta / 2}\right)\right)}\right] \text {. }
\end{aligned}
$$

In addition, e.g., Proposition 3.4 in Cox et al. [1] (with $H=H, U=U, \mathbb{H}=\mathbb{H}$, $\lambda=\lambda, A=A, T=T, p=p, \gamma=0, \eta=\vartheta, F=\left(H \ni v \mapsto e^{A \kappa} F(v) \in H_{-\vartheta}\right)$, $B=\left(H \ni v \mapsto\left(U \ni u \mapsto e^{A \kappa} B(v) u \in H_{-\vartheta / 2}\right) \in H S\left(U, H_{-\vartheta / 2}\right)\right), W=W, X=X^{\kappa}$ in the notation of Proposition 3.4 in [11]) shows that

$$
\begin{aligned}
& \sup _{t \in[0, T]}\left\|\max \left\{1,\left\|X_{t}^{\kappa}\right\|_{H}\right\}\right\|_{L^{p}(\mathbb{P} ; \mathbb{R})} \leq \sqrt{2}\left\|\max \left\{1,\|\xi\|_{H}\right\}\right\|_{L^{p}(\mathbb{P} ; \mathbb{R})} \\
& \cdot \mathcal{E}_{(1-\vartheta)}\left[\frac{T^{(1-\vartheta)} \sqrt{2}\|F\|_{\text {Lip }^{0}\left(H, H_{-\vartheta}\right)}}{\sqrt{1-\vartheta}}+\sqrt{T^{(1-\vartheta)} p(p-1)}\|B\|_{\text {Lip }^{0}\left(H, H S\left(U_{, H} H_{-\vartheta / 2}\right)\right)}\right] .
\end{aligned}
$$

Combining this with (230) establishes (226). The proof of Proposition 4.1 is thus completed.

The next result, Corollary 4.2 below, is an immediate consequence of Proposition 4.1 above.

Corollary 4.2. Assume the setting in Section 4.1. Then for every $\varepsilon \in\left(0, \frac{1-\vartheta}{2}\right]$ there exists a real number $C \in \mathbb{R}$ such that for all $\kappa \in[0, \infty)$ it holds that

$$
\left\|X_{T}^{0}-X_{T}^{\kappa}\right\|_{L^{p}(\mathbb{P} ; H)} \leq C \cdot \kappa^{\left(\frac{1-\vartheta}{2}-\varepsilon\right)} .
$$

\section{Weak convergence for Galerkin approximations of SEEs}

In this section the weak convergence results in Proposition 5.2, Corollary 5.3, and Corollary 5.4 are proved. Roughly speaking, in the framework of Section 1.4 we establish in Proposition 5.2 below a rate for the weak approximation error

$$
\left|\mathbb{E}\left[\varphi\left(X_{T}^{\mathbb{H}}\right)\right]-\mathbb{E}\left[\varphi\left(X_{T}^{I}\right)\right]\right|,
$$

where $I \subseteq \mathbb{H}$ is a set, where $\varphi: H \rightarrow \mathbb{R}$ is a four times continuously Fréchet differentiable function with globally bounded derivatives, and where $X^{\mathbb{H}}:[0, T] \times \Omega \rightarrow H$ and $X^{I}:[0, T] \times \Omega \rightarrow P_{I}(H)$ are appropriate mild solution processes of the SEEs in (234). Here, $X^{I}:[0, T] \times \Omega \rightarrow P_{I}(H)$ is a spectral Galerkin approximation of $X^{\mathbb{H}}:[0, T] \times \Omega \rightarrow H$. 
We note that the drift nonlinearity $F$ and the diffusion nonlinearity $B$ of the SEE (234) are not mollified and may take values in negative interpolation spaces. The proof of Proposition 5.2 uses both Corollary [3.9 in Section 3 and Proposition 4.1 in Section 4. Corollary 5.3 follows from an application of Proposition 5.2. Moreover, Corollary 5.4 is an immediate consequence of Corollary 5.3 .

\subsection{Setting}

Assume the setting in Section 1.4. let $\varphi \in C_{b}^{4}(H, \mathbb{R}), \theta \in[0,1), F \in C_{b}^{4}\left(H, H_{-\theta}\right), B \in$ $C_{b}^{4}\left(H, H S\left(U, H_{-\theta / 2}\right)\right), \xi \in L^{4}\left(\left.\mathbb{P}\right|_{\mathcal{F}_{0}} ; H\right)$, let $\varsigma_{F, B} \in \mathbb{R}$ be the real number given by $\varsigma_{F, B}=$ $\max \left\{1,\|F\|_{C_{b}^{3}\left(H_{,} H_{-\theta}\right)}^{2},\|B\|_{C_{b}^{3}\left(H, H S\left(U_{, H_{-\theta} / 2}\right)\right.}^{4}\right\}$, let $X^{I}:[0, T] \times \Omega \rightarrow P_{I}(H), I \in \mathcal{P}(\mathbb{H})$, and $X^{\mathbb{H}, \kappa, x}:[0, T] \times \Omega \rightarrow H, \kappa \in[0, \infty), x \in H$, be up-to-modifications unique $\left(\mathcal{F}_{t}\right)_{t \in[0, T]^{-}}$ predictable stochastic processes which satisfy for all $I \in \mathcal{P}(\mathbb{H}), \kappa \in[0, \infty), x \in H$ that $\sup _{t \in[0, T]} \mathbb{E}\left[\left\|X_{t}^{I}\right\|_{H}^{4}+\left\|X_{t}^{\mathbb{H}, \kappa, x}\right\|_{H}^{4}\right]<\infty$ and which satisfy that for all $I \in \mathcal{P}(\mathbb{H}), \kappa \in[0, \infty)$, $x \in H, t \in[0, T]$ it holds $\mathbb{P}$-a.s. that

$$
\begin{gathered}
X_{t}^{I}=e^{A t} P_{I}(\xi)+\int_{0}^{t} e^{A(t-s)} P_{I} F\left(X_{s}^{I}\right) d s+\int_{0}^{t} e^{A(t-s)} P_{I} B\left(X_{s}^{I}\right) d W_{s}, \\
X_{t}^{\mathbb{H}, \kappa, x}=e^{A t} x+\int_{0}^{t} e^{A(\kappa+t-s)} F\left(X_{s}^{\mathbb{H}, \kappa, x}\right) d s+\int_{0}^{t} e^{A(\kappa+t-s)} B\left(X_{s}^{\mathbb{H}, \kappa, x}\right) d W_{s},
\end{gathered}
$$

let $u^{(\kappa)}:[0, T] \times H \rightarrow \mathbb{R}, \kappa \in(0, \infty)$, be the functions which satisfy for all $\kappa \in(0, \infty), t \in$ $[0, T], x \in H$ that $u^{(\kappa)}(t, x)=\mathbb{E}\left[\varphi\left(X_{T-t}^{\mathbb{H}, \kappa, x}\right)\right]$, and let $c_{\delta_{1}, \ldots, \delta_{k}}^{(\kappa)} \in[0, \infty], \delta_{1}, \ldots, \delta_{k} \in(-\infty, 0]$, $k \in\{1,2,3,4\}, \kappa \in(0, \infty)$, be the extended real numbers which satisfy for all $\kappa \in(0, \infty)$, $k \in\{1,2,3,4\}, \delta_{1}, \ldots, \delta_{k} \in(-\infty, 0]$ that

$$
c_{\delta_{1}, \ldots, \delta_{k}}^{(\kappa)}=\sup _{t \in[0, T)} \sup _{x \in H} \sup _{v_{1}, \ldots, v_{k} \in H \backslash\{0\}}\left[\frac{\left|\left(\frac{\partial^{k}}{\partial x^{k}} u^{(\kappa)}\right)(t, x)\left(v_{1}, \ldots, v_{k}\right)\right|}{(T-t)^{\left(\delta_{1}+\ldots+\delta_{k}\right)}\left\|v_{1}\right\|_{H_{\delta_{1}}} \cdot \ldots \cdot\left\|v_{k}\right\|_{H_{\delta_{k}}}}\right]
$$

(cf., e.g., item (ii) of Lemma 3.1).

\subsection{A weak convergence result}

The following result, Lemma 5.1, is a slightly modified version of Lemma 3.4. Lemma 5.1 provides an a priori estimate for the quantities in (236) which is uniform in the mollification parameter $\kappa \in(0, T]$.

Lemma 5.1. Assume the setting in Section 5.1. Then it holds for all $k \in\{1,2,3,4\}$, $\delta_{1}, \ldots, \delta_{k} \in(-1 / 2,0]$ with $\sum_{i=1}^{k} \delta_{i}>-1 / 2$ that $\sup _{\kappa \in(0, T]} c_{\delta_{1}, \ldots, \delta_{k}}^{(\kappa)}<\infty$.

Proof. Throughout this proof let $\phi_{\kappa}:[0, T] \times H \rightarrow \mathbb{R}, \kappa \in(0, T]$, be the functions which satisfy for all $\kappa \in(0, T], t \in[0, T], x \in H$ that $\phi_{\kappa}(t, x)=\mathbb{E}\left[\varphi\left(X_{t}^{\mathbb{H}, \kappa, x}\right)\right]$. Note that for all $\kappa \in(0, T], t \in[0, T], x \in H$ it holds that $u^{(\kappa)}(t, x)=\phi_{\kappa}(T-t, x)$. This, (235), and [1, Item (iv) of Corollary 4.2] (with $T=T, \eta=0, H=H, U=U, V=\mathbb{R}, W=W$, $A=A, n=4, \alpha=\theta, \beta=\theta / 2, F=F, B=B, \varphi=\varphi, k=k, \delta_{1}=-\delta_{1}, \ldots, \delta_{k}=-\delta_{k}$, for $\left(\delta_{1}, \ldots, \delta_{k}\right) \in\left\{\left(x_{1}, \ldots, x_{k}\right) \in(-1 / 2,0]^{k}: \sum_{i=1}^{k} x_{i}>-1 / 2\right\}, k \in\{1,2,3,4\}$ in the notation of Corollary 4.2 in [1]) imply that for all $k \in\{1,2,3,4\}, \delta_{1}, \ldots, \delta_{k} \in(-1 / 2,0]$ 
with $\sum_{i=1}^{k} \delta_{i}>-1 / 2$ it holds that

$$
\begin{aligned}
& \sup _{\kappa \in(0, T]} c_{\delta_{1}, \ldots, \delta_{k}}^{(\kappa)} \\
& =\sup _{\kappa \in(0, T]} \sup _{t \in[0, T)} \sup _{x \in H} \sup _{v_{1}, \ldots, v_{k} \in H \backslash\{0\}}\left[\frac{\left|\left(\frac{\partial^{k}}{\partial x^{k}} u^{(\kappa)}\right)(t, x)\left(v_{1}, \ldots, v_{k}\right)\right|}{(T-t)^{\left(\delta_{1}+\ldots+\delta_{k}\right)}\left\|v_{1}\right\|_{H_{\delta_{1}}} \cdot \ldots \cdot\left\|v_{k}\right\|_{H_{\delta_{k}}}}\right] \\
& =\sup _{\kappa \in(0, T]} \sup _{t \in[0, T)} \sup _{x \in H} \sup _{v_{1}, \ldots, v_{k} \in H \backslash\{0\}}\left[\frac{\left|\left(\frac{\partial^{k}}{\partial x^{k}} \phi_{\kappa}\right)(T-t, x)\left(v_{1}, \ldots, v_{k}\right)\right|}{(T-t)^{\left(\delta_{1}+\ldots+\delta_{k}\right)}\left\|v_{1}\right\|_{H_{\delta_{1}}} \cdot \ldots \cdot\left\|v_{k}\right\|_{H_{\delta_{k}}}}\right] \\
& =\sup _{\kappa, t \in(0, T]} \sup _{x \in H} \sup _{v_{1}, \ldots, v_{k} \in H \backslash\{0\}}\left[\frac{\left|\left(\frac{\partial^{k}}{\partial x^{k}} \phi_{\kappa}\right)(t, x)\left(v_{1}, \ldots, v_{k}\right)\right|}{t^{\left(\delta_{1}+\ldots+\delta_{k}\right)}\left\|v_{1}\right\|_{H_{\delta_{1}}} \cdot \ldots \cdot\left\|v_{k}\right\|_{H_{\delta_{k}}}}\right]<\infty .
\end{aligned}
$$

The proof of Lemma 5.1 is thus completed.

Proposition 5.2. Assume the setting in Section 5.1 and let $I \in \mathcal{P}(\mathbb{H}), \vartheta \in[0,1 / 2) \cap[0, \theta]$. Then it holds for all $r \in[0,1-\vartheta), \rho \in(0,1-\theta)$ that

$$
\begin{aligned}
& \left|\mathbb{E}\left[\varphi\left(X_{T}^{\mathbb{H}}\right)\right]-\mathbb{E}\left[\varphi\left(X_{T}^{I}\right)\right]\right| \\
& \leq \frac{18}{|\min \{1, T\}|^{(\theta-\vartheta)}} \max \left\{\left\|\operatorname{Id}_{H}\right\|_{L\left(H, H_{-1}\right)}, \frac{1}{\left\|\operatorname{Id}_{H}\right\|_{L\left(H, H_{-1}\right)}}\right\} \mathbb{E}\left[\max \left\{1,\|\xi\|_{H}^{4}\right\}\right]\left\|P_{\mathbb{H} \backslash I}\right\|_{L\left(H, H_{-1}\right)}^{\frac{r \rho}{\rho+(\theta-\vartheta)}} \\
& \cdot\left\{\left[\frac{T^{(1-\rho / 2-\theta)}\|F\|_{C_{b}^{1}(H, H-\theta)}}{(1-\rho / 2-\theta)}+\frac{\left.\left.\sqrt{T^{(1-\rho-\theta)}}\|B\|_{C_{b}^{1}(H, H S(U, H-\theta / 2}\right)\right)}{\sqrt{1-\rho-\theta}}\right]\|\varphi\|_{C_{b}^{1}(H, \mathbb{R})}+\frac{\varsigma_{F, B}}{T^{r}}\left[1+\frac{T^{(1-\vartheta)}}{(1-\vartheta-r)}\right]^{2}\right. \\
& \left.\cdot\left[\|\varphi\|_{C_{b}^{3}(H, \mathbb{R})}+\sup _{\kappa \in(0, T]}\left[c_{-\vartheta}^{(\kappa)}+c_{-\vartheta, 0}^{(\kappa)}+c_{-\vartheta, 0,0}^{(\kappa)}+c_{-\vartheta / 2,-\vartheta / 2}^{(\kappa)}+c_{-\vartheta / 2,-\vartheta / 2,0}^{(\kappa)}+c_{-\vartheta / 2,-\vartheta / 2,0,0}^{(\kappa)}\right]\right]\right\} \\
& \cdot\left|\mathcal{E}_{(1-\theta)}\left[\frac{T^{(1-\theta)} \sqrt{2}\|F\|_{C_{b}^{1}(H, H-\theta)}}{\sqrt{1-\theta}}+\sqrt{12 T^{(1-\theta)}}\|B\|_{C_{b}^{1}\left(H, H S\left(U, H_{-\theta / 2}\right)\right)}\right]\right|^{4}<\infty .
\end{aligned}
$$

Proof. Throughout this proof assume w.l.o.g. that $I \neq \mathbb{H}$. We intend to prove Proposition 5.2 through an application of Corollary 3.9. Corollary 3.9 assumes that the initial random variable of the considered SEE takes values in $H_{2} \subseteq H$. In Section 5.1 above we, however, merely assume that the initial random variable $\xi$ takes values in $H$. To overcome this difficulty, we mollify the initial random variable in an appropriate sense so that the assumptions of Corollary 3.9 are met and Corollary 3.9 can be applied. More formally, note that there exist up-to-modifications unique $\left(\mathcal{F}_{t}\right)_{t \in[0, T]}$-predictable stochastic processes $\hat{X}^{J, \kappa, \delta}:[0, T] \times \Omega \rightarrow H, \kappa, \delta \in[0, \infty), J \in \mathcal{P}(\mathbb{H})$, such that for all $J \in \mathcal{P}(\mathbb{H})$, $\kappa, \delta \in[0, \infty)$ it holds that $\sup _{t \in[0, T]} \mathbb{E}\left[\left\|\hat{X}_{t}^{J, \kappa, \delta}\right\|_{H}^{4}\right]<\infty$ and such that for all $J \in \mathcal{P}(\mathbb{H})$, $\kappa, \delta \in[0, \infty), t \in[0, T]$ it holds $\mathbb{P}$-a.s. that

$$
\hat{X}_{t}^{J, \kappa, \delta}=e^{A(\delta+t)} P_{J}(\xi)+\int_{0}^{t} e^{A(\kappa+t-s)} P_{J} F\left(\hat{X}_{s}^{J, \kappa, \delta}\right) d s+\int_{0}^{t} e^{A(\kappa+t-s)} P_{J} B\left(\hat{X}_{s}^{J, \kappa, \delta}\right) d W_{s}
$$

In the next step we observe that the triangle inequality ensures that for all $\kappa, \delta \in(0, \infty)$ it holds that

$$
\begin{aligned}
& \left|\mathbb{E}\left[\varphi\left(\hat{X}_{T}^{\mathbb{H}, 0, \delta}\right)\right]-\mathbb{E}\left[\varphi\left(\hat{X}_{T}^{I, 0, \delta}\right)\right]\right| \leq\left|\mathbb{E}\left[\varphi\left(\hat{X}_{T}^{\mathbb{H}, 0, \delta}\right)\right]-\mathbb{E}\left[\varphi\left(\hat{X}_{T}^{\mathbb{H}, \kappa, \delta}\right)\right]\right| \\
& +\left|\mathbb{E}\left[\varphi\left(\hat{X}_{T}^{\mathbb{H}, \kappa, \delta}\right)\right]-\mathbb{E}\left[\varphi\left(\hat{X}_{T}^{I, \kappa, \delta}\right)\right]\right|+\left|\mathbb{E}\left[\varphi\left(\hat{X}_{T}^{I, \kappa, \delta}\right)\right]-\mathbb{E}\left[\varphi\left(\hat{X}_{T}^{I, 0, \delta}\right)\right]\right| .
\end{aligned}
$$

In the following we bound the three summands on the right hand side of (240). For the first and third summands on the right hand side of (240) we observe that Proposition 4.1 
shows that for all $\kappa, \delta \in(0, \infty), \rho \in[0,1-\theta)$ it holds that

$$
\begin{aligned}
& \left|\mathbb{E}\left[\varphi\left(\hat{X}_{T}^{\mathbb{H}, 0, \delta}\right)\right]-\mathbb{E}\left[\varphi\left(\hat{X}_{T}^{\mathbb{H}, \kappa, \delta}\right)\right]\right|+\left|\mathbb{E}\left[\varphi\left(\hat{X}_{T}^{I, \kappa, \delta}\right)\right]-\mathbb{E}\left[\varphi\left(\hat{X}_{T}^{I, 0, \delta}\right)\right]\right| \\
& \left.\left.\leq 4\|\varphi\|_{C_{b}^{1}(H, \mathbb{R})} \mid \mathcal{E}_{(1-\theta)}\left[\frac{T^{(1-\theta)} \sqrt{2}\|F\|_{C_{b}^{1}\left(H, H_{-\theta}\right)}}{\sqrt{1-\theta}}+\sqrt{2 T^{(1-\theta)}}\|B\|_{C_{b}^{1}\left(H, H S\left(U, H_{-} \theta / 2\right.\right.}\right)\right)\right]\left.\right|^{2} \\
& \cdot\left[\frac{T^{(1-\rho / 2-\theta)}}{(1-\rho / 2-\theta)}\|F\|_{C_{b}^{1}\left(H, H_{-\theta}\right)}+\frac{\sqrt{T^{(1-\rho-\theta)}}}{\sqrt{1-\rho-\theta}}\|B\|_{C_{b}^{1}\left(H, H S\left(U_{,} H_{-\theta / 2}\right)\right)}\right]\left\|\max \left\{1,\|\xi\|_{H}\right\}\right\|_{L^{2}(\mathbb{P} ; \mathbb{R})} \kappa^{\frac{\rho}{2}} .
\end{aligned}
$$

Next we bound the second summand on the right hand side of (240). For this we note that for all $\kappa \in(0, \infty)$ it holds that

$$
\max \left\{1,\left\|e^{\kappa A} F(\cdot)\right\|_{C_{b}^{3}\left(H, H_{-\vartheta}\right)}^{2},\left\|e^{\kappa A} B(\cdot)\right\|_{C_{b}^{3}\left(H, H S\left(U, H_{-}, 2\right)\right.}^{4}\right\} \leq \varsigma_{F, B} \max \left\{1, \kappa^{-2(\theta-\vartheta)}\right\} .
$$

This and Corollary 3.9 show that for all $\kappa, \delta \in(0, \infty), r \in[0,1-\vartheta)$ it holds that

$$
\begin{aligned}
\mid \mathbb{E} & {\left[\varphi\left(\hat{X}_{T}^{\mathbb{H}, \kappa, \delta}\right)\right]-\mathbb{E}\left[\varphi\left(\hat{X}_{T}^{I, \kappa, \delta}\right)\right] \mid } \\
\leq & {\left[1+\frac{T^{(1-\vartheta)}}{(1-\vartheta-r)}\right]^{2}\left|\mathcal{E}_{(1-\theta)}\left[\frac{T^{(1-\theta)} \sqrt{2}\|F\|_{C_{b}^{1}(H, H-\theta)}}{\sqrt{1-\theta}}+\sqrt{12 T^{(1-\theta)}}\|B\|_{C_{b}^{1}\left(H, H S\left(U, H_{-\theta / 2}\right)\right)}\right]\right|^{4} } \\
& \cdot\left[\|\varphi\|_{C_{b}^{3}(H, \mathbb{R})}+c_{-\vartheta}^{(\kappa)}+c_{-\vartheta, 0}^{(\kappa)}+c_{-\vartheta, 0,0}^{(\kappa)}+c_{-\vartheta / 2,-\vartheta / 2}^{(\kappa)}+c_{-\vartheta / 2,-\vartheta / 2,0}^{(\kappa)}+c_{-\vartheta / 2,-\vartheta / 2,0,0}^{(\kappa)}\right] \\
& \cdot \frac{18 \varsigma_{F, B}}{T^{r}} \mathbb{E}\left[\max \left\{1,\|\xi\|_{H}^{4}\right\}\right] \max \left\{1, \kappa^{-2(\theta-\vartheta)}\right\}\left\|P_{\mathbb{H} \backslash I}\right\|_{L(H, H-r)} .
\end{aligned}
$$

In the next step we plug (241) and (243) into (240) and we use the fact that $\forall r \in(0, \infty)$ : $\left\|P_{I}\right\|_{L\left(H, H_{-r}\right)}=\left\|P_{I}\right\|_{L\left(H, H_{-1}\right)}^{r}$ to obtain that for all $\kappa, \delta \in(0, \infty), r \in[0,1-\vartheta), \rho \in[0,1-\theta)$ it holds that

$$
\begin{aligned}
& \left|\mathbb{E}\left[\varphi\left(\hat{X}_{T}^{\mathbb{H}, 0, \delta}\right)\right]-\mathbb{E}\left[\varphi\left(\hat{X}_{T}^{I, 0, \delta}\right)\right]\right| \leq \max \left\{4 \kappa^{\frac{\rho}{2}}, 18 \max \left\{1, \kappa^{-2(\theta-\vartheta)}\right\}\left\|P_{\mathbb{H} \backslash I}\right\|_{L\left(H, H_{-1}\right)}^{r}\right\} \\
& \left.\cdot\left\{\left[\frac{T^{(1-\rho / 2-\theta)}}{(1-\rho / 2-\theta)}\|F\|_{C_{b}^{1}\left(H, H_{-\theta}\right)}+\frac{\sqrt{T^{(1-\rho-\theta)}}}{\sqrt{1-\rho-\theta}}\|B\|_{C_{b}^{1}\left(H, H S\left(U, H_{-} \theta / 2\right.\right.}\right)\right)\right]\|\varphi\|_{C_{b}^{1}(H, \mathbb{R})}+\frac{\varsigma_{F, B}}{T^{r}}\left[1+\frac{T^{(1-\vartheta)}}{(1-\vartheta-r)}\right]^{2} \\
& \left.\cdot\left[\|\varphi\|_{C_{b}^{3}(H, \mathbb{R})}+c_{-\vartheta}^{(\kappa)}+c_{-\vartheta, 0}^{(\kappa)}+c_{-\vartheta, 0,0}^{(\kappa)}+c_{-\vartheta / 2,-\vartheta / 2}^{(\kappa)}+c_{-\vartheta / 2,-\vartheta / 2,0}^{(\kappa)}+c_{-\vartheta / 2,-\vartheta / 2,0,0}^{(\kappa)}\right]\right\} \\
& \cdot \mathbb{E}\left[\max \left\{1,\|\xi\|_{H}^{4}\right\}\right]\left|\mathcal{E}_{(1-\theta)}\left[\frac{T^{(1-\theta)} \sqrt{2}\|F\|_{C_{b}^{1}\left(H, H_{-\theta}\right)}}{\sqrt{1-\theta}}+\sqrt{12 T^{(1-\theta)}}\|B\|_{C_{b}^{1}\left(H, H S\left(U, H_{-\theta / 2}\right)\right)}\right]\right|^{4} .
\end{aligned}
$$

Next we use the fact that $\left\|P_{\mathbb{H} \backslash I}\right\|_{L\left(H, H_{-1}\right)} \leq\left\|\operatorname{Id}_{H}\right\|_{L\left(H, H_{-1}\right)}$ to obtain that for all $r \in[0, \infty)$, $\rho \in(0,1-\theta)$ it holds that

$$
\begin{aligned}
& \inf _{\kappa \in(0, T]} \max \left\{4 \kappa^{\frac{\rho}{2}}, 18 \max \left\{1, \kappa^{-2(\theta-\vartheta)}\right\}\left\|P_{\mathbb{H} \backslash I}\right\|_{L\left(H, H_{-1}\right)}^{r}\right\} \\
\leq & \max \left\{4\left[\min \{1, T\}\left|\frac{\left.\left\|P_{\mathbb{H} \backslash I}\right\|_{L(H, H-1}\right)}{\left.\left\|\operatorname{Id}_{H}\right\|_{L(H, H-1}\right)}\right|^{\frac{2 r}{(\rho+4(\theta-\vartheta))}}\right]^{\frac{\rho}{2}},\right. \\
& \left.18 \max \left\{1,\left[\min \{1, T\}\left|\frac{\left.\left\|P_{\mathbb{H} \backslash I}\right\|_{L(H, H-1}\right)}{\left.\left\|\operatorname{Id}_{H}\right\|_{L(H, H}\right)}\right|^{\frac{2 r}{(\rho+4(\theta-\vartheta))}}\right]^{-2(\theta-\vartheta)}\right\}\left\|P_{\mathbb{H} \backslash I}\right\|_{L\left(H, H_{-1}\right)}^{r}\right\} \\
= & \max \left\{4\left[\min \{1, T\}\left|\frac{\left.\left\|P_{\mathbb{H} \backslash I}\right\|_{L(H, H-1}\right)}{\left\|\operatorname{Id}_{H}\right\|_{L\left(H, H_{-1}\right)}}\right|^{\frac{2 r}{(\rho+4(\theta-\vartheta))}}\right]^{\frac{\rho}{2}},\right.
\end{aligned}
$$




$$
\begin{aligned}
& \left.18\left\|P_{\mathbb{H} \backslash I}\right\|_{L\left(H, H_{-1}\right)}^{r}\left[\min \{1, T\}\left|\frac{\left\|P_{\mathbb{H} \backslash I}\right\|_{L\left(H, H_{-1}\right)}}{\left.\left\|\operatorname{Id}_{H}\right\|_{L(H, H-1}\right)}\right|^{\frac{2 r}{(\rho+4(\theta-\vartheta))}}\right]^{-2(\theta-\vartheta)}\right\}
\end{aligned}
$$

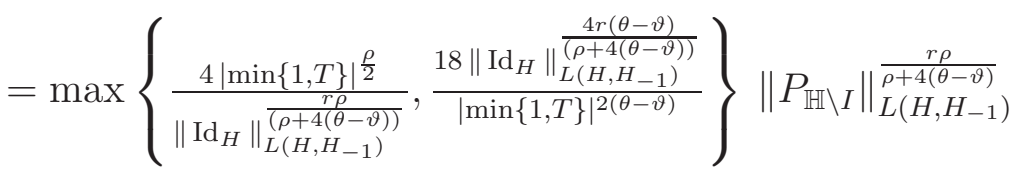

$$
\begin{aligned}
& \leq 18 \max \left\{\frac{1}{\min \left\{1,\left\|\operatorname{Id}_{H}\right\|_{L\left(H, H_{-1}\right)}^{r}\right\}}, \frac{\max \left\{1,\left\|\operatorname{Id}_{H}\right\|_{L\left(H, H_{-1}\right)}^{r}\right\}}{|\min \{1, T\}|^{2(\theta-\vartheta)}}\right\}\left\|P_{\mathbb{H} \backslash I}\right\|_{L\left(H, H_{-1}\right)}^{\frac{r \rho}{\rho+4(\theta-\vartheta)}} \\
& \leq \frac{18}{|\min \{1, T\}|^{2(\theta-\vartheta)}} \max \left\{\left\|\operatorname{Id}_{H}\right\|_{L\left(H, H_{-1}\right)}^{r}, \frac{1}{\left.\left\|\operatorname{Id}_{H}\right\|_{L(H, H-1}^{r}\right)}\right\}\left\|P_{\mathbb{H} \backslash I}\right\|_{L\left(H, H_{-1}\right)}^{\frac{r \rho}{\rho+(\theta-\vartheta)}} .
\end{aligned}
$$

Putting (245) into (244) implies that for all $\delta \in(0, \infty), r \in[0,1-\vartheta), \rho \in(0,1-\theta)$ it holds that

$$
\begin{aligned}
& \left|\mathbb{E}\left[\varphi\left(\hat{X}_{T}^{\mathbb{H}, 0, \delta}\right)\right]-\mathbb{E}\left[\varphi\left(\hat{X}_{T}^{I, 0, \delta}\right)\right]\right| \\
& \leq \frac{18}{|\min \{1, T\}|^{2(\theta-\vartheta)}} \max \left\{\left\|\operatorname{Id}_{H}\right\|_{L\left(H, H_{-1}\right)}^{r}, \frac{1}{\left.\left\|\operatorname{Id}_{H}\right\|_{L(H, H-1}^{r}\right)}\right\}\left\|P_{\mathbb{H} \backslash I}\right\|_{L(H, H-1)}^{\frac{r \rho}{\rho+4(\theta-\vartheta)}} \\
& \cdot\left\{\left[\frac{T^{(1-\rho / 2-\theta)}}{(1-\rho / 2-\theta)}\|F\|_{C_{b}^{1}\left(H, H_{-\theta}\right)}+\frac{\sqrt{T^{(1-\rho-\theta)}}}{\sqrt{1-\rho-\theta}}\|B\|_{C_{b}^{1}\left(H, H S\left(U, H_{-\theta / 2}\right)\right)}\right]\|\varphi\|_{C_{b}^{1}(H, \mathbb{R})}+\frac{\varsigma_{F, B}}{T^{r}}\left[1+\frac{T^{(1-\vartheta)}}{(1-\vartheta-r)}\right]^{2}\right. \\
& \left.\cdot\left[\|\varphi\|_{C_{b}^{3}(H, \mathbb{R})}+\sup _{\kappa \in(0, T]}\left[c_{-\vartheta}^{(\kappa)}+c_{-\vartheta, 0}^{(\kappa)}+c_{-\vartheta, 0,0}^{(\kappa)}+c_{-\vartheta / 2,-\vartheta / 2}^{(\kappa)}+c_{-\vartheta / 2,-\vartheta / 2,0}^{(\kappa)}+c_{-\vartheta / 2,-\vartheta / 2,0,0}^{(\kappa)}\right]\right]\right\} \\
& \cdot \mathbb{E}\left[\max \left\{1,\|\xi\|_{H}^{4}\right\}\right]\left|\mathcal{E}_{(1-\theta)}\left[\frac{T^{(1-\theta)} \sqrt{2}\|F\|_{C_{b}^{1}(H, H-\theta)}}{\sqrt{1-\theta}}+\sqrt{12 T^{(1-\theta)}}\|B\|_{C_{b}^{1}\left(H, H S\left(U, H_{-\theta / 2}\right)\right)}\right]\right|^{4} \cdot
\end{aligned}
$$

Moreover, we note that (234), (239), and Corollary 2.8 in [2] (with $H=H, U=U$, $T=T, \eta=0, p=2, \alpha=\theta, \hat{\alpha}=0, \beta=\theta / 2, \hat{\beta}=0, L_{0}=|F|_{\operatorname{Lip}^{0}\left(H_{,} H_{-\theta}\right)}, \hat{L}_{0}=$ $\|F(0)\|_{H_{-\theta}}, L_{1}=|B|_{\operatorname{Lip}^{0}\left(H, H S\left(U, H_{-\theta / 2}\right)\right)}, \hat{L}_{1}=\|B(0)\|_{H S\left(U, H_{-\theta / 2}\right)}, W=W, A=A, \mathbf{F}=$ $\left([0, T] \times \Omega \times H \ni(t, \omega, v) \mapsto P_{J} F(v) \in H_{-\theta}\right), \mathbf{B}=([0, T] \times \Omega \times H \ni(t, \omega, v) \mapsto(U \ni$ $\left.\left.u \mapsto P_{J} B(v) u \in H_{-\theta / 2}\right) \in H S\left(U, H_{-\vartheta / 2}\right)\right), \delta=0, X^{1}=\hat{X}^{J, 0, \delta}, X^{2}=\hat{X}^{J, 0,0}, \lambda=0$ for $J \in \mathcal{P}(\mathbb{H})$ in the notation of Corollary 2.8 in [2]) ensure that for all $J \in \mathcal{P}(\mathbb{H})$ it holds that $\lim _{(0, \infty) \ni \delta \rightarrow 0} \mathbb{E}\left[\varphi\left(\hat{X}_{T}^{J, 0, \delta}\right)\right]=\mathbb{E}\left[\varphi\left(\hat{X}_{T}^{J, 0,0}\right)\right]=\mathbb{E}\left[\varphi\left(X_{T}^{J}\right)\right]$. Combining this with inequality (246) proves the first inequality in (238). The second inequality in (238) follows from Lemma 5.1. The proof of Proposition 5.2 is thus completed.

In a number of cases the difference $\theta-\vartheta \geq 0$ can be chosen to be an arbitrarily small positive real number (cf. Theorem 1.1 above). In the next result, Corollary [5.3, we further estimate the right hand side of (238).

Corollary 5.3. Assume the setting in Section 5.1 and let $I \in \mathcal{P}(\mathbb{H}) \backslash\{\mathbb{H}\}, \vartheta \in[0,1 / 2) \cap$ $[0, \theta]$. Then it holds for all $\rho \in(0,1-\theta) \cap(4(\theta-\vartheta), \infty)$ that

$$
\begin{aligned}
& \left|\mathbb{E}\left[\varphi\left(X_{T}^{\mathbb{H}}\right)\right]-\mathbb{E}\left[\varphi\left(X_{T}^{I}\right)\right]\right| \\
& \leq \frac{18}{|\min \{1, T\}|^{2(\theta-\vartheta)}} \max \left\{\left\|\operatorname{Id}_{H}\right\|_{L\left(H, H_{-2}\right)}, \frac{1}{\left.\left\|\operatorname{Id}_{H}\right\|_{L(H, H-2}\right)}\right\}\left[\inf _{b \in \mathbb{H} \backslash I}\left|\lambda_{b}\right|\right]^{-(\rho-4(\theta-\vartheta))} \\
& \cdot\left\{\left[\frac{T^{(1-\rho / 2-\theta)}\|F\|_{C_{b}^{1}\left(H, H_{-\theta}\right)}}{(1-\rho / 2-\theta)}+\frac{\left.\sqrt{T^{(1-\rho-\theta)}}\|B\|_{C_{b}^{1}(H, H S(U, H-\theta / 2}\right)}{\sqrt{1-\rho-\theta}}\right]\|\varphi\|_{C_{b}^{1}(H, \mathbb{R})}+\frac{\varsigma_{F, B}}{T^{\rho}}\left[1+\frac{T^{(1-\vartheta)}}{(1-\vartheta-\rho)}\right]^{2}\right. \\
& \left.\cdot\left[\|\varphi\|_{C_{b}^{3}(H, \mathbb{R})}+\sup _{\kappa \in(0, T]}\left[c_{-\vartheta}^{(\kappa)}+c_{-\vartheta, 0}^{(\kappa)}+c_{-\vartheta, 0,0}^{(\kappa)}+c_{-\vartheta / 2,-\vartheta / 2}^{(\kappa)}+c_{-\vartheta / 2,-\vartheta / 2,0}^{(\kappa)}+c_{-\vartheta / 2,-\vartheta / 2,0,0}^{(\kappa)}\right]\right]\right\}
\end{aligned}
$$


$\cdot \mathbb{E}\left[\max \left\{1,\|\xi\|_{H}^{4}\right\}\right]\left|\mathcal{E}_{(1-\theta)}\left[\frac{T^{(1-\theta)} \sqrt{2}\|F\|_{C_{b}^{1}\left(H, H_{-\theta}\right)}}{\sqrt{1-\theta}}+\sqrt{12 T^{(1-\theta)}}\|B\|_{C_{b}^{1}\left(H, H S\left(U, H_{-\theta / 2}\right)\right)}\right]\right|^{4}<\infty$

Proof. First of all, we choose $r=\rho$ in (238) in Proposition 5.2 above to obtain that for all $\rho \in(0,1-\theta)$ it holds that

$$
\begin{aligned}
& \left|\mathbb{E}\left[\varphi\left(X_{T}^{\mathbb{H}}\right)\right]-\mathbb{E}\left[\varphi\left(X_{T}^{I}\right)\right]\right| \\
& \leq \frac{18}{|\min \{1, T\}|^{2(\theta-\vartheta)}} \max \left\{\left\|\operatorname{Id}_{H}\right\|_{L\left(H, H_{-1}\right)}, \frac{1}{\left.\left\|\operatorname{Id}_{H}\right\|_{L(H, H-1}\right)}\right\} \mathbb{E}\left[\max \left\{1,\|\xi\|_{H}^{4}\right\}\right]\left\|P_{\mathbb{H} \backslash I}\right\|_{L\left(H, H_{-1}\right)}^{\frac{\rho^{2}}{\rho+4(\theta-\vartheta)}} \\
& \cdot\left\{\left[\frac{T^{(1-\rho / 2-\theta)}\|F\|_{C_{b}^{1}\left(H, H_{-\theta}\right)}}{(1-\rho / 2-\theta)}+\frac{\left.\sqrt{T^{(1-\rho-\theta)}}\|B\|_{C_{b}^{1}(H, H S(U, H-\theta / 2}\right)}{\sqrt{1-\rho-\theta}}\right]\|\varphi\|_{C_{b}^{1}(H, \mathbb{R})}+\frac{\varsigma F, B}{T^{\rho}}\left[1+\frac{T^{(1-\vartheta)}}{(1-\vartheta-\rho)}\right]^{2}\right. \\
& \left.\cdot\left[\|\varphi\|_{C_{b}^{3}(H, \mathbb{R})}+\sup _{\kappa \in(0, T]}\left[c_{-\vartheta}^{(\kappa)}+c_{-\vartheta, 0}^{(\kappa)}+c_{-\vartheta, 0,0}^{(\kappa)}+c_{-\vartheta / 2,-\vartheta / 2}^{(\kappa)}+c_{-\vartheta / 2,-\vartheta / 2,0}^{(\kappa)}+c_{-\vartheta / 2,-\vartheta / 2,0,0}^{(\kappa)}\right]\right]\right\} \\
& \cdot\left|\mathcal{E}_{(1-\theta)}\left[\frac{T^{(1-\theta)} \sqrt{2}\|F\|_{C_{b}^{1}(H, H-\theta)}}{\sqrt{1-\theta}}+\sqrt{12 T^{(1-\theta)}}\|B\|_{C_{b}^{1}\left(H, H S\left(U_{,} H_{-\theta / 2}\right)\right)}\right]\right|^{4}<\infty .
\end{aligned}
$$

Next we note that for all $\rho \in(0,1-\theta) \cap(4(\theta-\vartheta), \infty)$ it holds that

$$
\begin{aligned}
& \left\|P_{\mathbb{H} \backslash I}\right\|_{L(H, H-1)}^{\frac{\rho^{2}}{\rho+4(\theta-\vartheta)}}=\left\|P_{\mathbb{H} \backslash I}\right\|_{L(H, H-1)}^{\rho\left[\frac{1}{1+4(\theta-\vartheta) / \rho}-1+\frac{4(\theta-\vartheta)}{\rho}\right]}\left\|P_{\mathbb{H} \backslash I}\right\|_{L\left(H, H_{-1}\right)}^{\rho\left[1-\frac{4(\theta-\vartheta)}{\rho}\right]} \\
& \leq\left|\max \left\{1,\left\|P_{\mathbb{H} \backslash I}\right\|_{L\left(H, H_{-1}\right)}\right\}\right|^{\rho\left[\frac{1}{1+4(\theta-\vartheta) / \rho}-1+\frac{4(\theta-\vartheta)}{\rho}\right]}\left\|P_{\mathbb{H} \backslash I}\right\|_{L\left(H, H_{-1}\right)}^{(\rho-4(\theta-\vartheta))} \\
& \leq \max \left\{1,\left\|P_{\mathbb{H} \backslash I}\right\|_{L\left(H, H_{-1}\right)}^{\rho}\right\}\left\|P_{\mathbb{H} \backslash I}\right\|_{L\left(H, H_{-1}\right)}^{(\rho-4(\theta-\vartheta))} \leq \max \left\{1,\left\|\operatorname{Id}_{H}\right\|_{L\left(H, H_{-1}\right)}\right\}\left\|P_{\mathbb{H} \backslash I}\right\|_{L\left(H, H_{-1}\right)}^{(\rho-4(\theta-\vartheta))}
\end{aligned}
$$

Combining this with (248), the fact that $\left\|\operatorname{Id}_{H}\right\|_{L\left(H, H_{-1}\right)}^{2}=\left\|\operatorname{Id}_{H}\right\|_{L\left(H, H_{-2}\right)}$, and the fact that $\left\|P_{\mathbb{H} \backslash I}\right\|_{L\left(H, H_{-1}\right)}^{(\rho-4(\theta-\vartheta))}=\left[\inf _{b \in \mathbb{H} \backslash I}\left|\lambda_{b}\right|\right]^{-(\rho-4(\theta-\vartheta))}$ completes the proof of Corollary 5.3,

The next result, Corollary 5.4 below, is an immediate consequence of Corollary 5.3 above.

Corollary 5.4. Assume the setting in Section 5.1 and assume that $\theta<3 / 5$. Then for every $\varepsilon \in(0, \infty)$ there exists a real number $C \in \mathbb{R}$ such that for all $I \in \mathcal{P}(\mathbb{H}) \backslash\{\mathbb{H}\}$ it holds that

$$
\left|\mathbb{E}\left[\varphi\left(X_{T}^{\mathbb{H}}\right)\right]-\mathbb{E}\left[\varphi\left(X_{T}^{I}\right)\right]\right| \leq C \cdot\left[\inf _{b \in \mathbb{H} \backslash I}\left|\lambda_{b}\right|\right]^{\varepsilon-(1-\theta-4 \max \{\theta-1 / 2,0\})} .
$$

Proof. Applying Corollary 5.3 (with $I=I, \vartheta=\min \left\{\theta, \frac{1}{2}\right\}-\frac{\varepsilon}{8} \mathbb{1}_{[1 / 2,1)}(\theta), \rho=1-\theta-$ $\frac{\varepsilon}{2}\left(2-\mathbb{1}_{[1 / 2,1)}(\theta)\right)$ for $\varepsilon \in\left(0,1-\theta-4 \max \left\{\theta-\frac{1}{2}, 0\right\}\right), I \in \mathcal{P}(\mathbb{H}) \backslash\{\mathbb{H}\}$ in the notation of Corollary 5.3) yields that for all $\varepsilon \in\left(0,1-\theta-4 \max \left\{\theta-\frac{1}{2}, 0\right\}\right)$ there exists a real number $C \in \mathbb{R}$ such that for all $I \in \mathcal{P}(\mathbb{H}) \backslash\{\mathbb{H}\}$ it holds that

$$
\left|\mathbb{E}\left[\varphi\left(X_{T}^{\mathbb{H}}\right)\right]-\mathbb{E}\left[\varphi\left(X_{T}^{I}\right)\right]\right| \leq C \cdot\left[\inf _{b \in \mathbb{H} \backslash I}\left|\lambda_{b}\right|\right]^{\varepsilon-(1-\theta-4 \max \{\theta-1 / 2,0\})} .
$$

This implies that for all $\varepsilon \in(0, \infty), \epsilon \in\left(0, \min \left\{1-\theta-4 \max \left\{\theta-\frac{1}{2}, 0\right\}, \varepsilon\right\}\right)$ there exists 
a real number $C \in[0, \infty)$ such that for all $I \in \mathcal{P}(\mathbb{H}) \backslash\{\mathbb{H}\}$ it holds that

$$
\begin{aligned}
\left|\mathbb{E}\left[\varphi\left(X_{T}^{\mathbb{H}}\right)\right]-\mathbb{E}\left[\varphi\left(X_{T}^{I}\right)\right]\right| & \leq C \cdot\left[\inf _{b \in \mathbb{H} \backslash I}\left|\lambda_{b}\right|\right]^{\epsilon-(1-\theta-4 \max \{\theta-1 / 2,0\})} \\
& =C \cdot\left[\inf _{b \in \mathbb{H} \backslash I}\left|\lambda_{b}\right|\right]^{(\epsilon-\varepsilon)} \cdot\left[\inf _{b \in \mathbb{H} \backslash I}\left|\lambda_{b}\right|\right]^{\varepsilon-(1-\theta-4 \max \{\theta-1 / 2,0\})} \\
& =\frac{C}{\left[\inf _{b \in \mathbb{H} \backslash I}\left|\lambda_{b}\right|\right]^{(\varepsilon-\epsilon)}} \cdot\left[\inf _{b \in \mathbb{H} \backslash I}\left|\lambda_{b}\right|\right]^{\varepsilon-(1-\theta-4 \max \{\theta-1 / 2,0\})} \\
& \leq\left[\frac{C}{\left[\inf _{b \in \mathbb{H}}\left|\lambda_{b}\right|\right]^{(\varepsilon-\epsilon)}}\right] \cdot\left[\inf _{b \in \mathbb{H} \backslash I}\left|\lambda_{b}\right|\right]^{\varepsilon-(1-\theta-4 \max \{\theta-1 / 2,0\})}
\end{aligned}
$$

This and the assumption that $\sup _{b \in \mathbb{H}} \lambda_{b}<0$ establish (250). The proof of Corollary 5.4 is thus completed.

\section{Weak convergence rates for SEEs}

In this section our main weak convergence result is established, see Corollary 6.1 below. Corollary 6.1 follows from an application of Corollary 5.4. Theorem 1.1 in the introductory section is an immediate consequence of Corollary 6.1.

Corollary 6.1. Let $\left(H,\langle\cdot, \cdot\rangle_{H},\|\cdot\|_{H}\right)$ and $\left(U,\langle\cdot, \cdot\rangle_{U},\|\cdot\|_{U}\right)$ be separable $\mathbb{R}$-Hilbert spaces, let $T \in(0, \infty), \iota \in[0,1 / 4], \gamma \in[0,1 / 2]$, let $\left(\Omega, \mathcal{F}, \mathbb{P},\left(\mathcal{F}_{t}\right)_{t \in[0, T]}\right)$ be a stochastic basis, let $\left(W_{t}\right)_{t \in[0, T]}$ be an $\operatorname{Id}_{U}$-cylindrical $\left(\Omega, \mathcal{F}, \mathbb{P},\left(\mathcal{F}_{t}\right)_{t \in[0, T]}\right)$-Wiener process, let $\left(e_{n}\right)_{n \in \mathbb{N}} \subseteq H$ be an orthonormal basis of $H$, let $\left(\lambda_{n}\right)_{n \in \mathbb{N}} \subseteq(0, \infty)$ be an increasing sequence, let $A: D(A) \subseteq$ $H \rightarrow H$ be a closed linear operator which satisfies $D(A)=\left\{v \in H: \sum_{n \in \mathbb{N}}\left|\lambda_{n}\left\langle e_{n}, v\right\rangle_{H}\right|^{2}<\right.$ $\infty\}$ and $\forall n \in \mathbb{N}: A e_{n}=-\lambda_{n} e_{n}$, let $\left(H_{r},\langle\cdot, \cdot\rangle_{H_{r}},\|\cdot\|_{H_{r}}\right), r \in \mathbb{R}$, be a family of interpolation spaces associated to $-A$, let $\xi \in H_{\iota}, \varphi \in C\left(H_{\iota}, \mathbb{R}\right), F \in C\left(H_{\iota}, H_{\iota-1}\right)$, $B \in C\left(H_{\iota}, H S\left(U, H_{\iota-1 / 2}\right)\right)$ satisfy for all $\varepsilon \in(0, \infty)$ that $\left(H_{\iota} \ni v \mapsto \varphi(v) \in \mathbb{R}\right),\left(H_{\iota} \ni\right.$ $\left.v \mapsto F(v) \in H_{\iota-\gamma-\varepsilon}\right)$, and $\left(H_{\iota} \ni v \mapsto\left(U \ni u \mapsto B(v) u \in H_{\iota-\gamma / 2-\varepsilon}\right) \in H S\left(U, H_{\iota-\gamma / 2-\varepsilon}\right)\right)$ are four times continuously Fréchet differentiable with globally bounded derivatives, let $\left(P_{N}\right)_{N \in \mathbb{N}} \subseteq L\left(H_{-1}\right)$ satisfy for all $N \in \mathbb{N}, v \in H$ that $P_{N}(v)=\sum_{n=1}^{N}\left\langle e_{n}, v\right\rangle_{H} e_{n}$, and let $X:[0, T] \times \Omega \rightarrow H_{\iota}$ and $X^{N}:[0, T] \times \Omega \rightarrow P_{N}(H), N \in \mathbb{N}$, be continuous $\left(\mathcal{F}_{t}\right)_{t \in[0, T]^{-}}$ adapted stochastic processes which satisfy that for all $N \in \mathbb{N}, t \in[0, T]$ it holds $\mathbb{P}$-a.s. that

$$
X_{t}=e^{A t} \xi+\int_{0}^{t} e^{A(t-s)} F\left(X_{s}\right) d s+\int_{0}^{t} e^{A(t-s)} B\left(X_{s}\right) d W_{s}
$$

and

$$
X_{t}^{N}=e^{A t} P_{N}(\xi)+\int_{0}^{t} e^{A(t-s)} P_{N} F\left(X_{s}^{N}\right) d s+\int_{0}^{t} e^{A(t-s)} P_{N} B\left(X_{s}^{N}\right) d W_{s} .
$$

Then for every $\varepsilon \in(0, \infty)$ there exists a real number $C \in \mathbb{R}$ such that for all $N \in \mathbb{N}$ it holds that

$$
\left|\mathbb{E}\left[\varphi\left(X_{T}\right)\right]-\mathbb{E}\left[\varphi\left(X_{T}^{N}\right)\right]\right| \leq C \cdot\left(\lambda_{N}\right)^{-(1-\gamma-\varepsilon)} .
$$

Proof. Throughout this proof let $\tilde{A}: D(\tilde{A}) \subseteq H_{\iota} \rightarrow H_{\iota}$ be the linear operator which satisfies $D(\tilde{A})=\left\{v \in H_{\iota}: \sum_{n \in \mathbb{N}}\left|\lambda_{n}\left\langle(-A)^{-\iota} e_{n}, v\right\rangle_{H_{\iota}}\right|^{2}<\infty\right\}$ and $\forall v \in D(\tilde{A}): \tilde{A} v=$ $\sum_{n \in \mathbb{N}}-\lambda_{n}\left\langle(-A)^{-\iota} e_{n}, v\right\rangle_{H_{\iota}}(-A)^{-\iota} e_{n}$, and let $\left(\tilde{H}_{r},\langle\cdot, \cdot\rangle_{\tilde{H}_{r}},\|\cdot\|_{\tilde{H}_{r}}\right), r \in \mathbb{R}$, be $\mathbb{R}$-Hilbert spaces which satisfy for all $r \in \mathbb{R}$ that

$$
\left(\tilde{H}_{r},\langle\cdot, \cdot\rangle_{\tilde{H}_{r}},\|\cdot\|_{\tilde{H}_{r}}\right)=\left(H_{\iota+r},\langle\cdot, \cdot\rangle_{H_{\iota+r}},\|\cdot\|_{H_{\iota+r}}\right) .
$$


Observe that $\left(\tilde{H}_{r},\langle\cdot, \cdot\rangle_{\tilde{H}_{r}},\|\cdot\|_{\tilde{H}_{r}}\right), r \in \mathbb{R}$, is a family of interpolation spaces associated to $-\tilde{A}$ (cf., e.g., [44, Section 3.7]). Moreover, note that for all $v \in H_{\iota-1}, t \in[0, T]$ it holds that

$$
e^{A t} v=e^{\tilde{A} t} v
$$

This, (253), and (254) imply that for all $N \in \mathbb{N}, t \in[0, T]$ it holds $\mathbb{P}$-a.s. that

$$
X_{t}=e^{\tilde{A} t} \xi+\int_{0}^{t} e^{\tilde{A}(t-s)} F\left(X_{s}\right) d s+\int_{0}^{t} e^{\tilde{A}(t-s)} B\left(X_{s}\right) d W_{s}
$$

and

$$
X_{t}^{N}=e^{\tilde{A} t} P_{N}(\xi)+\int_{0}^{t} e^{\tilde{A}(t-s)} P_{N} F\left(X_{s}^{N}\right) d s+\int_{0}^{t} e^{\tilde{A}(t-s)} P_{N} B\left(X_{s}^{N}\right) d W_{s} .
$$

Corollary 5.4 (with $H=H_{\iota}, U=U, T=T, W=W, \mathbb{H}=\left\{(-A)^{-\iota} e_{n}: n \in \mathbb{N}\right\}$, $\lambda\left((-A)^{-\iota} e_{N}\right)=-\lambda_{N}, A=\tilde{A}, H_{r}=\tilde{H}_{r}, P_{\left\{(-A)^{-\iota} e_{1}, \ldots,(-A)^{-\iota} e_{N}\right\}}(w)=P_{N}(w), \varphi=\varphi$, $\theta=\gamma+\varepsilon / 10, F=\left(\tilde{H}_{0} \ni v \mapsto F(v) \in \tilde{H}_{-\gamma-\varepsilon / 10}\right), B=\left(\tilde{H}_{0} \ni v \mapsto(U \ni u \mapsto B(v) u \in\right.$ $\left.\left.\tilde{H}_{-\gamma / 2-\varepsilon / 20}\right) \in H S\left(U, \tilde{H}_{-\gamma / 2-\varepsilon / 20}\right)\right), \xi=\left(\Omega \ni \omega \mapsto \xi \in \tilde{H}_{0}\right), X^{\mathbb{H}}=X, X^{\left\{(-A)^{-\iota} e_{1}, \ldots,(-A)^{-\iota} e_{N}\right\}}=$ $X^{N}, \varepsilon=\frac{9 \varepsilon}{10}-\frac{2 \varepsilon}{5} \mathbb{1}_{\{1 / 2\}}(\gamma)$ for $\varepsilon \in\left(0,5-10 \gamma+\mathbb{1}_{\{1 / 2\}}(\gamma)\right), w \in H_{\iota-1}, r \in \mathbb{R}, N \in \mathbb{N}$ in the notation of Corollary 5.4) therefore ensures that for all $\varepsilon \in\left(0,5-10 \gamma+\mathbb{1}_{\{1 / 2\}}(\gamma)\right)$ there exists a real number $C \in[0, \infty)$ such that for all $N \in \mathbb{N}$ it holds that

$$
\begin{aligned}
\left|\mathbb{E}\left[\varphi\left(X_{T}\right)\right]-\mathbb{E}\left[\varphi\left(X_{T}^{N}\right)\right]\right| & \leq C \cdot\left[\inf _{\left\{e_{n}: n \in\{N+1, N+2, \ldots\}\right\}} \lambda_{n}\right]^{-(1-\gamma-\varepsilon)} \\
& \leq C \cdot\left(\lambda_{N}\right)^{-(1-\gamma-\varepsilon)}
\end{aligned}
$$

This and the fact that $\left(\lambda_{n}\right)_{n \in \mathbb{N}} \subseteq(0, \infty)$ is an increasing sequence imply that for all $\varepsilon \in(0, \infty), \epsilon \in\left(0, \min \left\{5-10 \gamma+\mathbb{1}_{\{1 / 2\}}(\gamma), \varepsilon\right\}\right)$ there exists a real number $C \in[0, \infty)$ such that for all $N \in \mathbb{N}$ it holds that

$$
\begin{aligned}
\left|\mathbb{E}\left[\varphi\left(X_{T}\right)\right]-\mathbb{E}\left[\varphi\left(X_{T}^{N}\right)\right]\right| & \leq C \cdot\left(\lambda_{N}\right)^{-(1-\gamma-\epsilon)} \\
& =C \cdot\left(\lambda_{N}\right)^{-(\varepsilon-\epsilon)} \cdot\left(\lambda_{N}\right)^{-(1-\gamma-\varepsilon)} \\
& =\frac{C}{\left(\lambda_{N}\right)^{(\varepsilon-\epsilon)}} \cdot\left(\lambda_{N}\right)^{-(1-\gamma-\varepsilon)} \\
& \leq\left[\frac{C}{\left(\lambda_{1}\right)^{(\varepsilon-\epsilon)}}\right] \cdot\left(\lambda_{N}\right)^{-(1-\gamma-\varepsilon)} .
\end{aligned}
$$

The proof of Corollary 6.1 is thus completed.

\section{Lower bounds for the weak error of Galerkin ap- proximations for SEEs}

In this section a few specific lower bounds for weak approximation errors are established in the case of concrete examples of SEEs. More precisely, in this section we provide lower bounds for weak approximation errors for spatial spectral Galerkin approximations of linear stochastic heat equations with vanishing drift nonlinearities $F=0$. Lower bounds for strong approximation errors for example SEEs and whole classes of SEEs can be found in [17, 38, 40, 41]. In the case of finite dimensional stochastic ordinary differential equations, lower bounds for both strong and weak approximation errors have been established; for details see, e.g., the references in the overview article Müller-Gronbach \& Ritter [39]. 


\section{1 $\quad$ Setting}

Assume the setting in Section 1.4, assume that $\left(H,\langle\cdot, \cdot\rangle_{H},\|\cdot\|_{H}\right)=\left(U,\langle\cdot, \cdot\rangle_{U},\|\cdot\|_{U}\right)$, let $\beta \in[0,1 / 2)$, let $\mu: \mathbb{H} \rightarrow \mathbb{R}$ be a function such that $\sum_{b \in \mathbb{H}}\left|\mu_{b}\right|^{2}\left|\lambda_{b}\right|^{-2 \beta}<\infty$, and let $B \in H S\left(H, H_{-\beta}\right)$ satisfy that for all $v \in H$ it holds that $B v=\sum_{b \in \mathbb{H}} \mu_{b}\langle b, v\rangle_{H} b$.

The above assumptions ensure that there exist up-to-modifications unique $\left(\mathcal{F}_{t}\right)_{t \in[0, T]^{-}}$ predictable stochastic processes $X^{I}:[0, T] \times \Omega \rightarrow H, I \in \mathcal{P}(\mathbb{H})$, such that for all $p \in$ $(0, \infty), I \in \mathcal{P}(\mathbb{H})$ it holds that $\sup _{t \in[0, T]} \mathbb{E}\left[\left\|X_{t}^{I}\right\|_{H}^{p}\right]<\infty$ and such that for all $I \in \mathcal{P}(\mathbb{H})$, $t \in[0, T]$ it holds $\mathbb{P}$-a.s. that $X_{t}^{I}=\int_{0}^{t} e^{A(t-s)} P_{I} B d W_{s}$.

\subsection{Lower bounds for the weak error}

Lemma 7.1. Assume the setting in Section 7.1 and let $I \in \mathcal{P}(\mathbb{H}), b \in \mathbb{H}, t \in[0, T]$. Then $\operatorname{Var}\left(\left\langle b, X_{t}^{I}\right\rangle_{H}\right)=\frac{\mathbb{1}_{I}(b)\left|\mu_{b}\right|^{2}\left(e^{2 \lambda_{b} t}-1\right)}{2 \lambda_{b}}$.

Proof. Observe that it holds $\mathbb{P}$-a.s. that

$$
\begin{aligned}
& \left\langle b, X_{t}^{I}\right\rangle_{H}=\left\langle b, \int_{0}^{t} e^{A(t-s)} P_{I} B d W_{s}\right\rangle_{H}=\int_{0}^{t}\left\langle P_{I} e^{A(t-s)} b, B d W_{s}\right\rangle_{H} \\
& =\mathbb{1}_{I}(b) \int_{0}^{t} e^{\lambda_{b}(t-s)}\left\langle b, B d W_{s}\right\rangle_{H}=\mathbb{1}_{I}(b) \mu_{b} \int_{0}^{t} e^{\lambda_{b}(t-s)}\left\langle b, d W_{s}\right\rangle_{H} .
\end{aligned}
$$

This and Itô's isometry yield that

$$
\operatorname{Var}\left(\left\langle b, X_{t}^{I}\right\rangle_{H}\right)=\mathbb{1}_{I}(b)\left|\mu_{b}\right|^{2} \int_{0}^{t} e^{2 \lambda_{b}(t-s)} d s=\frac{\mathbb{1}_{I}(b)\left|\mu_{b}\right|^{2}\left(e^{2 \lambda_{b} t}-1\right)}{2 \lambda_{b}} .
$$

The proof of Lemma 7.1 is thus completed.

The next elementary result, Lemma 7.2 , is an immediate consequence of Lemma 7.1 above.

Lemma 7.2. Assume the setting in Section 7.1, let $I \in \mathcal{P}(\mathbb{H})$, and let $\varphi: H \rightarrow[0, \infty)$ fulfill that for all $x \in H$ it holds that $\varphi(x)=\|x\|_{H}^{2}$. Then $\varphi \in C^{\infty}(H,[0, \infty))$ and

$$
\mathbb{E}\left[\varphi\left(X_{T}^{\mathbb{H}}\right)\right]-\mathbb{E}\left[\varphi\left(X_{T}^{I}\right)\right]=\mathbb{E}\left[\left\|X_{T}^{\mathbb{H} \backslash I}\right\|_{H}^{2}\right] \geq\left[\frac{1-e^{-2 T \inf _{b \in \mathbb{H}}\left|\lambda_{b}\right|}}{2}\right]\left[\sum_{b \in \mathbb{H} \backslash I} \frac{\left|\mu_{b}\right|^{2}}{\left|\lambda_{b}\right|}\right] .
$$

Lemma 7.2 establishes a lower bound in the case of the squared norm as the test function. The next result, Lemma 7.3, establishes a similar lower bound for a test function in $C_{b}^{4}(H, \mathbb{R})$.

Lemma 7.3. Assume the setting in Section 7.1, let $I \in \mathcal{P}(\mathbb{H})$ and let $\varphi: H \rightarrow \mathbb{R}$ be given by $\varphi(v)=\exp \left(-\|v\|_{H}^{2}\right)$ for all $v \in H$. Then $\varphi \in C_{b}^{4}(H, \mathbb{R})$ and

$$
\mathbb{E}\left[\varphi\left(X_{T}^{I}\right)\right]-\mathbb{E}\left[\varphi\left(X_{T}^{\mathbb{H}}\right)\right] \geq \frac{\mathbb{E}\left[\varphi\left(X_{T}^{\mathbb{H}}\right)\right] \mathbb{E}\left[\left\|X_{T}^{\mathbb{M} \backslash I}\right\|_{H}^{2}\right]}{2\left(1+\mathbb{E}\left[\left\|X_{T}^{\mathbb{M} \backslash I}\right\|_{H}^{2}\right]\right)^{3 / 2}} \geq \frac{\mathbb{E}\left[\varphi\left(X_{T}^{\mathbb{H}}\right)\right]\left(1-e^{-2 T \inf _{b \in \mathbb{H}}\left|\lambda_{b}\right|}\right)}{4\left(1+\mathbb{E}\left[\left\|X_{T}^{\mathbb{H} \backslash I}\right\|_{H}^{2}\right]\right)^{3 / 2}}\left[\sum_{b \in \mathbb{H} \backslash I} \frac{\left|\mu_{b}\right|^{2}}{\left|\lambda_{b}\right|}\right] .
$$

Proof. First of all, observe that for all $x, u_{1}, u_{2}, u_{3}, u_{4} \in H$ it holds that

$$
\begin{aligned}
\varphi^{(1)}(x)\left(u_{1}\right)= & -2 \varphi(x)\left\langle x, u_{1}\right\rangle_{H} \\
\varphi^{(2)}(x)\left(u_{1}, u_{2}\right)= & -2\left[\varphi^{(1)}(x)\left(u_{2}\right)\left\langle x, u_{1}\right\rangle_{H}+\varphi(x)\left\langle u_{2}, u_{1}\right\rangle_{H}\right] \\
= & -2 \varphi(x)\left[\left\langle u_{1}, u_{2}\right\rangle_{H}-2\left\langle x, u_{1}\right\rangle_{H}\left\langle x, u_{2}\right\rangle_{H}\right], \\
\varphi^{(3)}(x)\left(u_{1}, u_{2}, u_{3}\right)= & -2\left[\varphi^{(1)}(x)\left(\left\langle u_{3}, u_{1}\right\rangle_{H} u_{2}+\left\langle u_{2}, u_{1}\right\rangle_{H} u_{3}\right)\right. \\
& \left.+\varphi^{(2)}(x)\left(u_{2}, u_{3}\right)\left\langle x, u_{1}\right\rangle_{H}\right], \\
\varphi^{(4)}(x)\left(u_{1}, u_{2}, u_{3}, u_{4}\right)= & -2\left[\varphi^{(3)}(x)\left(u_{2}, u_{3}, u_{4}\right)\left\langle x, u_{1}\right\rangle_{H}+\varphi^{(2)}(x)\left(u_{2}, u_{3}\right)\left\langle u_{4}, u_{1}\right\rangle_{H}\right. \\
& \left.+\varphi^{(2)}(x)\left(\left\langle u_{3}, u_{1}\right\rangle_{H} u_{2}+\left\langle u_{2}, u_{1}\right\rangle_{H} u_{3}, u_{4}\right)\right] .
\end{aligned}
$$


Identity (265) and the fact that for all $r \in[0, \infty)$ it holds that $\sup _{x \in H}\left(\left[1+\|x\|_{H}^{r}\right] \varphi(x)\right)<$ $\infty$ show that for all $r \in[0, \infty)$ it holds that $\sup _{x \in H}\left(\left[1+\|x\|_{H}^{r}\right]\left[\varphi(x)+\left\|\varphi^{(1)}(x)\right\|_{L(H, \mathbb{R})}\right]\right)<$ $\infty$. This and identity (266) imply that for all $r \in[0, \infty)$ it holds that

$$
\sup _{x \in H}\left(\left[1+\|x\|_{H}^{r}\right]\left[\sum_{k=1}^{2}\left\|\varphi^{(k)}(x)\right\|_{L^{(k)}(H, \mathbb{R})}\right]\right)<\infty .
$$

This and (267) yield that for all $r \in[0, \infty)$ it holds that

$$
\sup _{x \in H}\left(\left[1+\|x\|_{H}^{r}\right]\left[\sum_{k=1}^{3}\left\|\varphi^{(k)}(x)\right\|_{L^{(k)}(H, \mathbb{R})}\right]\right)<\infty .
$$

This and (268) prove that $\varphi \in C_{b}^{4}(H, \mathbb{R})$. Next observe that for all $\sigma \in \mathbb{R}$ it holds that

$$
\begin{aligned}
& \int_{\mathbb{R}} \exp \left(-[\sigma x]^{2}\right) \cdot \frac{1}{\sqrt{2 \pi}} \exp \left(-\frac{x^{2}}{2}\right) d x=\int_{\mathbb{R}} \frac{1}{\sqrt{2 \pi}} \exp \left(-\frac{x^{2}}{2}\left[1+2 \sigma^{2}\right]\right) d x \\
& =\frac{1}{\left[1+2 \sigma^{2}\right]^{1 / 2}} \int_{\mathbb{R}} \frac{1}{\sqrt{2 \pi}} \exp \left(-\frac{x^{2}}{2}\right) d x=\frac{1}{\sqrt{1+2 \sigma^{2}}} .
\end{aligned}
$$

This and Lemma 7.1 imply that

$$
\begin{aligned}
& \mathbb{E}\left[\varphi\left(X_{T}^{I}\right)\right]-\mathbb{E}\left[\varphi\left(X_{T}^{\mathbb{H}}\right)\right] \\
& =\prod_{b \in I}\left[1+\frac{\left|\mu_{b}\right|^{2}}{\lambda_{b}}\left(e^{2 \lambda_{b} T}-1\right)\right]^{-1 / 2}-\prod_{b \in \mathbb{H}}\left[1+\frac{\left|\mu_{b}\right|^{2}}{\lambda_{b}}\left(e^{2 \lambda_{b} T}-1\right)\right]^{-1 / 2} \\
& =\prod_{b \in I}\left[1+\frac{\left|\mu_{b}\right|^{2}}{\lambda_{b}}\left(e^{2 \lambda_{b} T}-1\right)\right]^{-1 / 2}\left[1-\prod_{b \in \mathbb{H} \backslash I}\left[1+\frac{\left|\mu_{b}\right|^{2}}{\lambda_{b}}\left(e^{2 \lambda_{b} T}-1\right)\right]^{-1 / 2}\right] \\
& \geq \prod_{b \in \mathbb{H}}\left[1+\frac{\left|\mu_{b}\right|^{2}}{\lambda_{b}}\left(e^{2 \lambda_{b} T}-1\right)\right]^{-1 / 2}\left[1-\left[\prod_{b \in \mathbb{H} \backslash I}\left[1+\frac{\left|\mu_{b}\right|^{2}}{\lambda_{b}}\left(e^{2 \lambda_{b} T}-1\right)\right]\right]^{-1 / 2}\right] \\
& \geq \mathbb{E}\left[\varphi\left(X_{T}^{\mathbb{H}}\right)\right]\left[1-\left[1+\sum_{b \in \mathbb{H} \backslash I} \frac{\left|\mu_{b}\right|^{2}}{\lambda_{b}}\left(e^{2 \lambda_{b} T}-1\right)\right]^{-1 / 2}\right] .
\end{aligned}
$$

In the next step we note that the fundamental theorem of calculus ensures that for all $x \in[0, \infty)$ it holds that $1-[1+x]^{-1 / 2}=\frac{1}{2} \int_{0}^{x}[1+y]^{-3 / 2} d y \geq \frac{1}{2} x[1+x]^{-3 / 2}$. Combining this with (272) and Lemma 7.1 proves that

$$
\begin{aligned}
& \mathbb{E}\left[\varphi\left(X_{T}^{I}\right)\right]-\mathbb{E}\left[\varphi\left(X_{T}^{\mathbb{H}}\right)\right] \\
& \geq \frac{\mathbb{E}\left[\varphi\left(X_{T}^{\mathbb{H}}\right)\right]}{2}\left[\sum_{b \in \mathbb{H} \backslash I} \frac{\left|\mu_{b}\right|^{2}}{\lambda_{b}}\left(e^{2 \lambda_{b} T}-1\right)\right]\left[1+\sum_{b \in \mathbb{H} \backslash I} \frac{\left|\mu_{b}\right|^{2}}{\lambda_{b}}\left(e^{2 \lambda_{b} T}-1\right)\right]^{-3 / 2} \\
& \geq \frac{\mathbb{E}\left[\varphi\left(X_{T}^{\mathbb{H}}\right)\right] \mathbb{E}\left[\left\|X_{T}^{\mathbb{H} \backslash I}\right\|_{H}^{2}\right]}{2\left(1+\mathbb{E}\left[\left\|X_{T}^{\mathbb{H} \backslash I}\right\|_{H}^{2}\right]\right)^{3 / 2}} .
\end{aligned}
$$

This and Lemma 7.2 complete the proof of Lemma 7.3 .

Proposition 7.4 (A more concrete lower bound for the weak error). Assume the setting in Section 7.1, let $b: \mathbb{N} \rightarrow \mathbb{H}$ be a bijective function, let $I \in \mathcal{P}(\mathbb{H}), N \in \mathbb{N}, c, \rho \in(0, \infty)$, $\delta \in\left(-\infty, \frac{1}{2}-\frac{1}{2 \rho}\right)$ satisfy for all $n \in \mathbb{N}$ that $\lambda_{b_{n}}=-c n^{\rho}$ and $\mu_{b_{n}}=\left|\lambda_{b_{n}}\right|^{\delta}$, and let $\varphi: H \rightarrow \mathbb{R}$ satisfy for all $v \in H$ that $\varphi(v)=\exp \left(-\|v\|_{H}^{2}\right)$. Then $\varphi \in C_{b}^{4}(H, \mathbb{R}), B \in$ $\cap_{r \in\left(-\infty,-\frac{1}{2}[1 / \rho+2 \delta]\right)} H S\left(H, H_{r}\right)$, and

$$
\mathbb{E}\left[\varphi\left(X_{T}^{\left\{b_{1}, \ldots, b_{N}\right\}}\right)\right]-\mathbb{E}\left[\varphi\left(X_{T}^{\mathbb{H}}\right)\right] \geq \frac{\mathbb{E}\left[\varphi\left(X_{T}^{\mathbb{H}}\right)\right]\left(1-e^{-2 T c}\right)\left|\lambda_{b_{N}}\right|^{-\left(1-\left[^{1} / \rho+2 \delta\right]\right)}}{2^{(1-2 \delta \rho+\rho)} c^{1 / \rho}\left(\rho-2 \delta \rho+c^{(2 \delta-1)}(\rho-2 \delta \rho-1)^{-1 / 3}\right)^{3 / 2}} .
$$


Proof. First of all, observe that Lemma 7.3 ensures that $\varphi \in C_{b}^{4}(H, \mathbb{R})$ and

$$
\mathbb{E}\left[\varphi\left(X_{T}^{\left\{b_{1}, \ldots, b_{N}\right\}}\right)\right]-\mathbb{E}\left[\varphi\left(X_{T}^{\mathbb{H}}\right)\right] \geq \frac{\mathbb{E}\left[\varphi\left(X_{T}^{\mathbb{H}}\right)\right]\left(1-e^{-2 T c}\right) c^{(2 \delta-1)}}{4\left(1+c^{(2 \delta-1)} \sum_{n=N+1}^{\infty} n^{\rho(2 \delta-1)}\right)^{3 / 2}}\left[\sum_{n=N+1}^{\infty} n^{\rho(2 \delta-1)}\right] .
$$

Next note that the assumption that $\delta<\frac{1}{2}-\frac{1}{2 \rho}$ ensures that $\rho(2 \delta-1)<-1$. This, in turn, implies that

$$
\begin{aligned}
& \sum_{n=N+1}^{\infty} n^{\rho(2 \delta-1)}=\sum_{n=N+1}^{\infty} \int_{n}^{n+1} \frac{1}{n^{\rho(1-2 \delta)}} d x \geq \sum_{n=N+1}^{\infty} \int_{n}^{n+1} \frac{1}{x^{\rho(1-2 \delta)}} d x \\
= & \int_{N+1}^{\infty} x^{\rho(2 \delta-1)} d x=\frac{-(N+1)^{[1+\rho(2 \delta-1)]}}{[1+\rho(2 \delta-1)]} \geq \frac{(2 N)^{\rho(1 / \rho+2 \delta-1)}}{[\rho(1-2 \delta)-1]} \\
= & \frac{\left[2^{\rho} / c\right]^{(1 / \rho+2 \delta-1)}\left|\lambda_{b_{N}}\right|^{(1 / \rho+2 \delta-1)}}{[\rho(1-2 \delta)-1]} .
\end{aligned}
$$

Putting this into (275) proves that

$$
\mathbb{E}\left[\varphi\left(X_{T}^{\left\{b_{1}, \ldots, b_{N}\right\}}\right)\right]-\mathbb{E}\left[\varphi\left(X_{T}^{\mathbb{H}}\right)\right] \geq \frac{\mathbb{E}\left[\varphi\left(X_{T}^{\mathbb{H}}\right)\right]\left(1-e^{-2 T c}\right) 2^{(1+2 \delta \rho-\rho)}\left|\lambda_{b_{N}}\right|^{(1 / \rho+2 \delta-1)}}{4 c^{1 / \rho}(\rho-2 \delta \rho-1)\left(1+c^{(2 \delta-1)} \sum_{n=N+1}^{\infty} n^{\rho(2 \delta-1)}\right)^{3 / 2}} .
$$

This and the fact that

$$
\begin{aligned}
& \sum_{n=N+1}^{\infty} n^{\rho(2 \delta-1)}=\sum_{n=N+1}^{\infty} \int_{n-1}^{n} \frac{1}{n^{\rho(1-2 \delta)}} d x \leq \sum_{n=N+1}^{\infty} \int_{n-1}^{n} \frac{1}{x^{\rho(1-2 \delta)}} d x \\
& =\int_{N}^{\infty} x^{\rho(2 \delta-1)} d x=\frac{-N^{[1+\rho(2 \delta-1)]}}{[1+\rho(2 \delta-1)]}=\frac{N^{(1+2 \delta \rho-\rho)}}{(\rho-2 \delta \rho-1)} \leq \frac{1}{(\rho-2 \delta \rho-1)}
\end{aligned}
$$

complete the proof of Proposition 7.4.

In the next result, Corollary 7.4, we specialize Proposition 7.4 to the case where $\rho=2$, $c=\pi^{2}$ (we think of $A$ being, e.g., the Laplacian with Dirichlet boundary conditions on $\left.H=L^{2}((0,1) ; \mathbb{R})\right)$ and $\delta \in(-\infty, 1 / 4)$.

Corollary 7.5. Assume the setting in Section 7.1, let $b: \mathbb{N} \rightarrow \mathbb{H}$ be a bijective function, let $I \in \mathcal{P}(\mathbb{H}), N \in \mathbb{N}, \delta \in(-\infty, 1 / 4)$ satisfy that for all $n \in \mathbb{N}$ it holds that $\lambda_{b_{n}}=-\pi^{2} n^{2}$ and $\mu_{b_{n}}=\left|\lambda_{b_{n}}\right|^{\delta}$, and let $\varphi: H \rightarrow \mathbb{R}$ satisfy for all $v \in H$ that $\varphi(v)=\exp \left(-\|v\|_{H}^{2}\right)$. Then $\varphi \in C_{b}^{4}(H, \mathbb{R}), B \in \cap_{r \in\left(-\infty,-\frac{1}{2}[1 / 2+2 \delta]\right)} H S\left(H, H_{r}\right)$, and

$$
\mathbb{E}\left[\varphi\left(X_{T}^{\left\{b_{1}, \ldots, b_{N}\right\}}\right)\right]-\mathbb{E}\left[\varphi\left(X_{T}^{\mathbb{H}}\right)\right] \geq\left[\frac{\mathbb{E}\left[\varphi\left(X_{T}^{\mathbb{H}}\right)\right] 2^{(4 \delta-5)}\left(1-e^{-T}\right)}{\left(2-4 \delta+2^{(7 \delta-7)}(1-4 \delta)^{-1 / 3}\right)^{3 / 2}}\right]\left|\lambda_{b_{N}}\right|^{-(1-[1 / 2+2 \delta])}
$$

\section{Acknowledgements}

Jarred Foster is gratefully acknowledged for a few helpful suggestions regarding English wording. Michael Schatz, Xiaojie Wang, and an anonymous referee are gratefully acknowledged for bringing a few typos into our notice. This project has been supported through the SNSF-Research project 200021_156603 "Numerical approximations of nonlinear stochastic ordinary and partial differential equations". 


\section{References}

[1] Andersson, A., Hefter, M., Jentzen, A., and Kurniawan, R. Regularity properties for solutions of infinite dimensional Kolmogorov equations in Hilbert spaces. arXiv:1611.00858 (2016), 36 pages. Minor revision requested from Potential Anal. (2017).

[2] Andersson, A., Jentzen, A., And Kurniawan, R. Existence, uniqueness, and regularity for stochastic evolution equations with irregular initial values. arXiv:1512.06899 (2016), 35 pages. Revision requested from the J. Math. Anal. Appl. (2017).

[3] Andersson, A., Jentzen, A., Kurniawan, R., And Welti, T. On the differentiability of solutions of stochastic evolution equations with respect to their initial values. arXiv:1611.00856 (2016), 41 pages. To appear in Nonlinear Anal. (2017).

[4] Andersson, A., Kruse, R., And Larsson, S. Duality in refined SobolevMalliavin spaces and weak approximation of SPDE. Stoch. Partial Differ. Equ. Anal. Comput. 4, 1 (2016), 113-149.

[5] Andersson, A., And Larsson, S. Weak convergence for a spatial approximation of the nonlinear stochastic heat equation. Math. Comp. 85, 299 (2016), 1335-1358.

[6] BrÉHier, C.-E. Approximation of the invariant measure with an Euler scheme for stochastic PDEs driven by space-time white noise. Potential Anal. 40, 1 (2014), 1-40.

[7] Bréhier, C.-E., And Kopec, M. Approximation of the invariant law of SPDEs: error analysis using a Poisson equation for a full-discretization scheme. IMA J. Numer. Anal. (2016).

[8] BrzeźniaK, Z. On stochastic convolution in Banach spaces and applications. Stochastics Stochastics Rep. 61, 3-4 (1997), 245-295.

[9] Brzeźniak, Z., van Neerven, J. M. A. M., Veraar, M. C., and Weis, L. Itô's formula in UMD Banach spaces and regularity of solutions of the Zakai equation. J. Differential Equations 245, 1 (2008), 30-58.

[10] Carmona, R. A., And Molchanov, S. A. Parabolic Anderson problem and intermittency. Mem. Amer. Math. Soc. 108, 518 (1994), viii+125.

[11] Cox, S., Hutzenthaler, M., Jentzen, A., van Neerven, J., and Welti, T. Convergence in Hölder norms with applications to Monte Carlo methods in infinite dimensions. arXiv:1605.00856 (2017), 48 pages. To appear in IMA J. Num. Anal. (2017).

[12] Cox, S., Jentzen, A., Kurniawan, R., And Pušnik, P. On the mild Itô formula in Banach spaces. arXiv:1612.03210 (2016), 27 pages. Minor revision requested from Discrete Contin. Dyn. Syst. Ser. B (2017).

[13] Da Prato, G. Kolmogorov equations for stochastic PDE's with multiplicative noise. In Stochastic analysis and applications, vol. 2 of Abel Symp. Springer, Berlin, 2007, pp. 235-263.

[14] Da Prato, G., Jentzen, A., and Röckner, M. A mild Ito formula for SPDEs. arXiv:1009.3526 (2012), 39 pages. To appear in Trans. Amer. Math. Soc. (2017). 
[15] Da Prato, G., And ZabczyK, J. Stochastic equations in infinite dimensions, vol. 44 of Encyclopedia of Mathematics and its Applications. Cambridge University Press, Cambridge, 1992.

[16] Da Prato, G., AND ZabcZYK, J. Stochastic equations in infinite dimensions, second ed., vol. 152 of Encyclopedia of Mathematics and its Applications. Cambridge University Press, Cambridge, 2014.

[17] Davie, A. M., And Gaines, J. G. Convergence of numerical schemes for the solution of parabolic stochastic partial differential equations. Math. Comp. 70, 233 (2001), 121-134.

[18] De BoundD, A., And Debussche, A. Weak and strong order of convergence of a semidiscrete scheme for the stochastic nonlinear Schrödinger equation. Appl. Math. Optim. 54, 3 (2006), 369-399.

[19] Debussche, A. Weak approximation of stochastic partial differential equations: the nonlinear case. Math. Comp. 80, 273 (2011), 89-117.

[20] Debussche, A., And Printems, J. Weak order for the discretization of the stochastic heat equation. Math. Comp. 78, 266 (2009), 845-863.

[21] Dörsek, P. Semigroup splitting and cubature approximations for the stochastic Navier-Stokes equations. SIAM J. Numer. Anal. 50, 2 (2012), 729-746.

[22] Geissert, M., Kovács, M., and Larsson, S. Rate of weak convergence of the finite element method for the stochastic heat equation with additive noise. BIT 49, 2 (2009), 343-356.

[23] Gorenflo, R., Kilbas, A. A., Mainardi, F., and Rogosin, S. V. MittagLeffler functions, related topics and applications. Springer Monographs in Mathematics. Springer, Heidelberg, 2014.

[24] Hausenblas, E. Weak approximation for semilinear stochastic evolution equations. In Stochastic analysis and related topics VIII, vol. 53 of Progr. Probab. Birkhäuser, Basel, 2003, pp. 111-128.

[25] Hausenblas, E. Weak approximation of the stochastic wave equation. J. Comput. Appl. Math. 235, 1 (2010), 33-58.

[26] Hefter, M., Jentzen, A., And Kurniawan, R. Counterexamples to regularities for the derivative processes associated to stochastic evolution equations. arXiv:1703.09198 (2017), 26 pages.

[27] Henry, D. Geometric theory of semilinear parabolic equations, vol. 840 of Lecture Notes in Mathematics. Springer-Verlag, Berlin-New York, 1981.

[28] Hutzenthaler, M., Jentzen, A., And Salimova, D. Strong convergence of fulldiscrete nonlinearity-truncated accelerated exponential Euler-type approximations for stochastic Kuramoto-Sivashinsky equations. arXiv:1604.02053 (2016), 43 pages.

[29] Jentzen, A., And Kurniawan, R. Weak convergence rates for Euler-type approximations of semilinear stochastic evolution equations with nonlinear diffusion coefficients. arXiv:1501.03539 (2015), 51 pages. 
[30] Jentzen, A., And Pušnik, P. Exponential moments for numerical approximations of stochastic partial differential equations. arXiv:1609.07031 (2016), 44 pages. Revision requested from Stoch. Partial Differ. Equ. Anal. Comput. (2017).

[31] Kloeden, P. E., And Platen, E. Numerical solution of stochastic differential equations, vol. 23 of Applications of Mathematics (New York). Springer-Verlag, Berlin, 1992.

[32] Kovács, M., Larsson, S., And Lindgren, F. Weak convergence of finite element approximations of linear stochastic evolution equations with additive noise. BIT 52, 1 (2012), 85-108.

[33] Kovács, M., Larsson, S., And Lindgren, F. Weak convergence of finite element approximations of linear stochastic evolution equations with additive noise II. Fully discrete schemes. BIT 53, 2 (2013), 497-525.

[34] Kruse, R. Strong and weak approximation of semilinear stochastic evolution equations, vol. 2093 of Lecture Notes in Mathematics. Springer, Cham, 2014.

[35] Lindgren, F. On weak and strong convergence of numerical approximations of stochastic partial differential equations. PhD thesis, Chalmers University of Technology and University of Gothenburg (2012), 60 pages.

[36] Lindner, F., And Schilling, R. L. Weak order for the discretization of the stochastic heat equation driven by impulsive noise. Potential Anal. 38, 2 (2013), $345-379$.

[37] Milstein, G. N. Numerical integration of stochastic differential equations, vol. 313 of Mathematics and its Applications. Kluwer Academic Publishers Group, Dordrecht, 1995. Translated and revised from the 1988 Russian original.

[38] Müller-Gronbach, T., And Ritter, K. Lower bounds and nonuniform time discretization for approximation of stochastic heat equations. Found. Comput. Math. 7, 2 (2007), 135-181.

[39] Müller-Gronbach, T., And Ritter, K. Minimal errors for strong and weak approximation of stochastic differential equations. In Monte Carlo and quasi-Monte Carlo methods 2006. Springer, Berlin, 2008, pp. 53-82.

[40] Müller-Gronbach, T., Ritter, K., and Wagner, T. Optimal pointwise approximation of a linear stochastic heat equation with additive space-time white noise. In Monte Carlo and quasi-Monte Carlo methods 2006. Springer, Berlin, 2008, pp. 577-589.

[41] Müller-Gronbach, T., Ritter, K., And Wagner, T. Optimal pointwise approximation of infinite-dimensional Ornstein-Uhlenbeck processes. Stoch. Dyn. 8, 3 (2008), 519-541.

[42] Renardy, M., And Rogers, R. C. An introduction to partial differential equations, vol. 13 of Texts in Applied Mathematics. Springer-Verlag, New York, 1993.

[43] Rössler, A. Runge-Kutta Methods for the Numerical Solution of Stochastic Differential Equations. Shaker Verlag, Aachen, 2003. Dissertation, Technische Universität Darmstadt, Darmstadt, Germany. 
[44] Sell, G. R., And You, Y. Dynamics of evolutionary equations, vol. 143 of Applied Mathematical Sciences. Springer-Verlag, New York, 2002.

[45] Shardlow, T. Weak convergence of a numerical method for a stochastic heat equation. BIT 43, 1 (2003), 179-193.

[46] van Neerven, J., Veraar, M., And Weis, L. Stochastic integration in Banach spaces - a survey. In Stochastic analysis: a series of lectures, vol. 68 of Progr. Probab. Birkhäuser/Springer, Basel, 2015, pp. 297-332.

[47] van Neerven, J. M. A. M., Veraar, M. C., And Weis, L. Stochastic evolution equations in UMD Banach spaces. J. Funct. Anal. 255, 4 (2008), 940-993.

[48] Wang, X. An exponential integrator scheme for time discretization of nonlinear stochastic wave equation. J. Sci. Comput. 64, 1 (2015), 234-263.

[49] WAng, X. Weak error estimates of the exponential Euler scheme for semi-linear SPDEs without Malliavin calculus. Discrete Contin. Dyn. Syst. 36, 1 (2016), 481497.

[50] WAng, X., AND GAn, S. Weak convergence analysis of the linear implicit Euler method for semilinear stochastic partial differential equations with additive noise. $J$. Math. Anal. Appl. 398, 1 (2013), 151-169. 